CERN-TH/97-257

UB-ECM-PF $97 / 26$

hep-th/9709180

September 1997

\title{
Duality in Quantum Field Theory (and String Theory) ${ }^{*}$
}

\author{
Luis Álvarez-Gaumé ${ }^{a}$ and Frederic Zamora ${ }^{b}$. \\ a Theory Division, CERN, \\ 1211 Geneva 23, Switzerland. \\ ${ }^{b}$ Departament d'Estructura i Constituents de la Materia, \\ Facultat de Física, Universitat de Barcelona, \\ Diagonal 647, E-08028 Barcelona, Spain.
}

\begin{abstract}
These lectures give an introduction to duality in Quantum Field Theory. We discuss the phases of gauge theories and the implications of the electric-magnetic duality transformation to describe the mechanism of confinement. We review the exact results of $N=1$ supersymmetric QCD and the Seiberg-Witten solution of $N=2$ super Yang-Mills. Some of its extensions to String Theory are also briefly discussed.
\end{abstract}

CERN-TH/97-257

UB-ECM-PF $97 / 26$

September 1997

\footnotetext{
*Based on a lectures delivered by L. A.-G. at The Workshop on Fundamental Particles and Interactions, held in Vanderbilt University, and at CERN-La Plata-Santiago de Compostela School of Physics, both in May 1997.
} 
XIII The vacuum structure of SQCD with

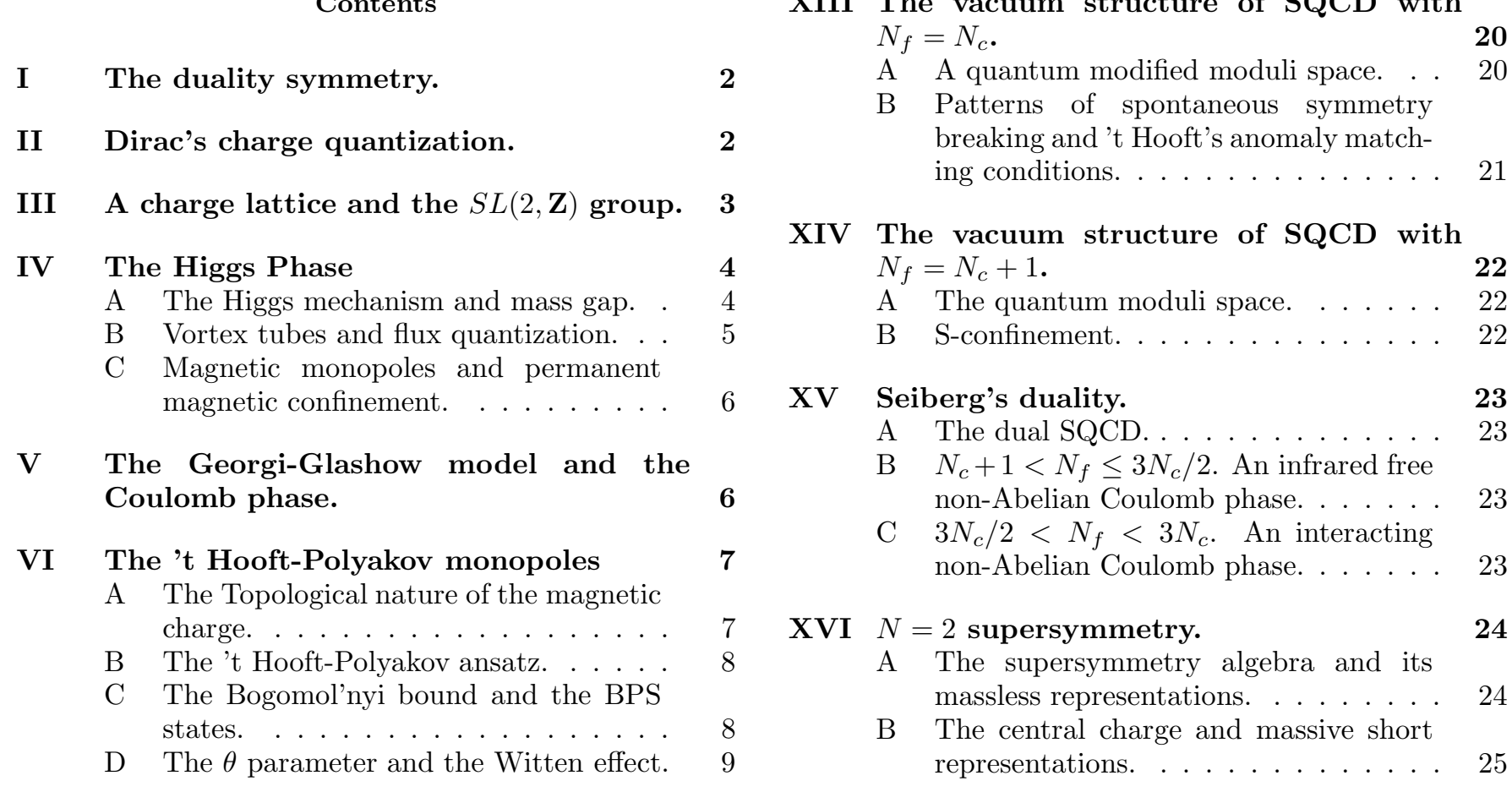

VII The Confining phase.

A The Abelian projection.

B The nature of the gauge singularities. . 10

C The phases of the Yang-Mills vacuum. 11

D Oblique confinement. ........ 11

VIII The Higgs/confining phase.

IX Supersymmetry

A The supersymmetry algebra and its massless representations. . . . . . . . 12

B Superspace and superfields _. . - 12

C Supersvmmetric Lagrangians, . . . . . 13

D R-symmetry. . . . . . . . . . 15

$\mathrm{X}$ The uses of supersymmetry.

A Flat directions and super-Higgs mecha-

\begin{tabular}{|cc|}
\hline & nism \\
\hline \hline B & Wilsonian effective actions and holo- \\
\hline \hline &
\end{tabular} morphy.

XI $N=1$ SQCD,

A Classical Lagrangian and symmetries. . 17

B The classical moduli space. . . . . . . . 18

XII The vacuum structure of SQCD with

$N_{f}<N_{c}$.

A The Afleck-Dine-Seiberg's superpotential. 19

B Massive flavors. . . . . . . . . . . 19
XVII $N=2 S U(2)$ super Yang-Mills theory in perturbation theory. 25

A The $N=2$ Lagrangian. . . . . . . . . . 25

B The flat direction. . . . . . . . . 26

\begin{tabular}{|ll}
\hline XVIII The low energy effective Lagrangian. 26 &
\end{tabular}

\begin{tabular}{|ll}
\hline XIX & BPS bound and duality. \\
\hline
\end{tabular}

\begin{tabular}{|l|l|}
\hline$X X$ & Singularities in the moduli space.
\end{tabular}

XXI The physical interpretation of the singularities.

XXII The Seiberg-Witten solution. 29

A The inputs. . . . . . . . . . . . 29

B $\quad$ The geometrical picture. . . . . . . . 29

C The Physical connection with $N=2$ super Yang-Mills. . . . . . . . . . 30

XXIII Breaking $N=2$ to $N=1$. Monopole condensation and confinement. $\quad 30$

XXIV $\quad$ Breaking $N=2$ to $N=0$. 31

XXV String Theory in perturbation theory. 33

A The type IIA and type IIB string theories. 34

B The Type I string theory. . . . . . . . 35

C The $S O(32)$ and $E_{8} \times E_{8}$ heterotic strings. 35

\begin{tabular}{|l|l|}
\hline XXVI D-branes. & 35 \\
\hline
\end{tabular}

A Dirichlet boundary conditions. . . . . . 35

B BPS states with RR charges. . . . . . . 36 
XXVII Some final comments on nonpertur\begin{tabular}{|ll}
\hline bative String Theory. & 37
\end{tabular}

A D-instantons and S-duality. . . . . . . . . 37

B An eleventh dimension. . . . . . . . . 37

\section{THE DUALITY SYMMETRY.}

From a historical point of view we can say that many of the fundamental concepts of twentieth century Physics have Maxwell's equations at its origin. In particular some of the symmetries that have led to our understanding of the fundamental interactions in terms of relativistic quantum field theories have their roots in the equations describing electromagnetism. As we will now describe, the most basic form of the duality symmetry also appears in the source free Maxwell equations:

$$
\begin{aligned}
\nabla \cdot(\mathbf{E}+i \mathbf{B}) & =0, \\
\frac{\partial}{\partial t}(\mathbf{E}+i \mathbf{B})+i \nabla \times(\mathbf{E}+i \mathbf{B}) & =0 .
\end{aligned}
$$

These equations are invariant under Lorentz transformations, and making all of Physics compatible with these symmetries led Einstein to formulate the Theory of Relativity. Other important symmetries of (I.1) are conformal and gauge invariance, which have later played important roles in our understanding of phase transitions and critical phenomena, and in the formulation of the fundamental interactions in terms of gauge theories. In these lectures however we will study the implications of yet another symmetry hidden in (I.1): duality. The simplest form of duality is the invariance of (I.1) under the interchange of electric and magnetic fields:

$$
\begin{aligned}
& \mathbf{B} \rightarrow \mathbf{E}, \\
& \mathbf{E} \rightarrow-\mathbf{B} .
\end{aligned}
$$

In fact, the vacuum Maxwell equations (I.1) admit a continuous $S O(2)$ transformation symmetry

$$
(\mathbf{E}+i \mathbf{B}) \rightarrow e^{i \phi}(\mathbf{E}+i \mathbf{B})
$$

If we include ordinary electric sources the equations (1.1) become:

$$
\begin{aligned}
\nabla \cdot(\mathbf{E}+i \mathbf{B}) & =q, \\
\frac{\partial}{\partial t}(\mathbf{E}+i \mathbf{B})+i \nabla \times(\mathbf{E}+i \mathbf{B}) & =\mathbf{j}_{e} .
\end{aligned}
$$

In presence of matter, the duality symmetry is not valid. To keep it, magnetic sources have to be introduced:

\footnotetext{
${ }^{\dagger}$ Notice that the duality transformations are not a symmetry of the electromagnetic action. Concerning this issue see [4].
}

$$
\begin{aligned}
\nabla \cdot(\mathbf{E}+i \mathbf{B}) & =(q+i g), \\
\frac{\partial}{\partial t}(\mathbf{E}+i \mathbf{B})+i \nabla \times(\mathbf{E}+i \mathbf{B}) & =\left(\mathbf{j}_{e}+i \mathbf{j}_{m}\right) .
\end{aligned}
$$

Now the duality symmetry is restored if at the same time we also rotate the electric and magnetic charges

$$
(q+i g) \rightarrow e^{i \phi}(q+i g) .
$$

The complete physical meaning of the duality symmetry is still not clear, but a lot of work has been dedicated in recent years to understand the implications of this type of symmetry. We will focus mainly on the applications to Quantum Field Theory. In the final sections, we will briefly review some of the applications to String Theory, where duality make striking an profound predictions.

\section{DIRAC'S CHARGE QUANTIZATION.}

From the classical point of view the inclusion of magnetic charges is not particularly problematic. Since the Maxwell equations, and the Lorentz equations of motion for electric and magnetic charges only involve the electric and magnetic field, the classical theory can accommodate any values for the electric and magnetic charges.

However, when we try to make a consistent quantum theory including monopoles, deep consequences are obtained. Dirac obtained his celebrated quantization condition precisely by studying the consistency conditions for a quantum theory in the presence of electric and magnetic charges [2]. We derive it here by the quantization of the angular momentum, since it allows to extend it to the case of dyons, i.e., particles that carry both electric and magnetic charges.

Consider a non-relativistic charge $q$ in the vicinity of a magnetic monopole of strength $g$, situated at the origin. The charge $q$ experiences a force $m \ddot{\vec{r}}=q \dot{\vec{r}} \times \vec{B}$, where $\vec{B}$ is the monopole field given by $\vec{B}=g \vec{r} / 4 \pi r^{3}$. The change in the orbital angular momentum of the electric charge under the effect of this force is given by

$$
\begin{aligned}
& \frac{d}{d t}(m \vec{r} \times \dot{\vec{r}})=m \vec{r} \times \ddot{\vec{r}} \\
& =\frac{q g}{4 \pi r^{3}} \vec{r} \times(\dot{\vec{r}} \times \vec{r})=\frac{d}{d t}\left(\frac{q g}{4 \pi} \frac{\vec{r}}{r}\right) .
\end{aligned}
$$

Hence, the total conserved angular momentum of the system is

$$
\vec{J}=\vec{r} \times m \dot{\vec{r}}-\frac{q g}{4 \pi} \frac{\vec{r}}{r} .
$$

The second term on the right hand side (henceforth denoted by $\vec{J}_{e m}$ ) is the contribution coming from the electromagnetic field. This term can be directly computed by using the fact that the momentum density of an electromagnetic field is given by its Poynting vector, $\vec{E} \times \vec{B}$, 
and hence its contribution to the angular momentum is given by

$$
\vec{J}_{e m}=\int d^{3} x \vec{r} \times(\vec{E} \times \vec{B})=\frac{g}{4 \pi} \int d^{3} x \vec{r} \times\left(\vec{E} \times \frac{\vec{r}}{r^{3}}\right) .
$$

In components,

$$
\begin{aligned}
J_{e m}^{i} & =\frac{g}{4 \pi} \int d^{3} x E^{j} \partial_{j}\left(\hat{x}^{i}\right) \\
& =\frac{g}{4 \pi} \int_{S^{2}} \hat{x}^{i} \vec{E} \cdot \overrightarrow{d s}-\frac{g}{4 \pi} \int d^{3} x(\vec{\nabla} \cdot \vec{E}) \hat{x}^{i} .
\end{aligned}
$$

When the separation between the electric and magnetic charges is negligible compared to their distance from the boundary $S^{2}$, the contribution of the first integral to $\vec{J}_{e m}$ vanishes by spherical symmetry. We are therefore left with

$$
\vec{J}_{e m}=-\frac{g q}{4 \pi} \hat{r}
$$

Returning to equation (II.2), if we assume that orbital angular momentum is quantized. Then it follows that

$$
\frac{q g}{4 \pi}=\frac{1}{2} n
$$

where $n$ is an integer. Equation (II.5) is the Dirac's charge quantization condition. It implies that if there exists a magnetic monopole of charge $g$ somewhere in the universe, then all electric charges are quantized in units of $2 \pi / g$. If we have a number of purely electric charges $q_{i}$ and purely magnetic charges $g_{j}$, then any pair of them will satisfy a quantization condition:

$$
q_{i} g_{j}=2 \pi n_{i j}
$$

Thus, any electric charge is an integral multiple of $2 \pi / g_{j}$. For a given $g_{j}$, let these charges have $n_{0 j}$ as the highest common factor. Then, all the electric charges are multiples of $q_{0}=n_{0 j} 2 \pi / g_{j}$. Similar considerations apply to the quantization of the magnetic charge.

Till now, we have only dealt with particles that carry either an electric or a magnetic charge. Consider now two dyons of charges $\left(q_{1}, g_{1}\right)$ and $\left(q_{2}, g_{2}\right)$. For this system, we can repeat the calculation of $\vec{J}_{e m}$ by following the steps in (II.3) where now the electromagnetic fields are split as $\vec{E}=\vec{E}_{1}+\vec{E}_{2}$ and $\vec{B}=\vec{B}_{1}+\vec{B}_{2}$. The answer is easily found to be

$$
\vec{J}_{e m}=-\frac{1}{4 \pi}\left(q_{1} g_{2}-q_{2} g_{1}\right) \hat{r}
$$

The charge quantization condition is thus generalized to

$$
\frac{q_{1} g_{2}-q_{2} g_{1}}{4 \pi}=\frac{1}{2} n_{12}
$$

This is referred to as the Dirac-Schwinger-Zwanziger condition [3].

\section{A CHARGE LATTICE AND THE $S L(2, \mathbf{Z})$ GROUP.}

In the previous section we derived the quantization of the electric charge of particles without magnetic charge, in terms of some smallest electric charge $q_{0}$. For a dyon $\left(q_{n}, g_{n}\right)$, this gives $q_{0} g_{n}=2 \pi n$. Thus, the smallest magnetic charge the dyon can have is $g_{0}=2 \pi m_{0} / q_{0}$, with $m_{0}$ a positive integer dependent on the detailed theory considered. For two dyons of the same magnetic charge $g_{0}$ and electric charges $q_{1}$ and $q_{2}$, the quantization condition implies $q_{1}-q_{2}=n q_{0}$, with $n$ a multiple of $m_{0}$. Therefore, although the difference of electric charges is quantized, the individual charges are still arbitrary. It introduces a new parameter $\theta$ that contributes to the electric charge of any dyon with magnetic charge $g_{0}$ by

$$
q=q_{0}\left(n_{e}+\frac{\theta}{2 \pi}\right)
$$

Observe that the parameter $\theta+2 \pi$ gives the same electric charges that the parameter $\theta$ by shifting $n_{e} \rightarrow n_{e}+1$. Thus, we look at the parameter $\theta$ as an angular variable.

This arbitrariness in the electric charge of dyons through the $\theta$ parameter can be fixed if the theory is $\mathrm{CP}$ invariant. Under a $\mathrm{CP}$ transformation $(q, g) \rightarrow(-q, g)$. If the theory is $\mathrm{CP}$ invariant, the existence of a state $\left(q, g_{0}\right)$ necessarily leads to the existence of $\left(-q, g_{0}\right)$. Applying the quantization condition to this pair, we get $2 q=q_{0} \times$ integer. This implies that $q=n q_{0}$ or $q=\left(n+\frac{1}{2}\right) q_{0}$. If $\theta \neq 0, \pi$, the theory is not CP invariant. It indicates that the $\theta$ parameter is a source of $\mathrm{CP}$ violation. Later on we will identify $\theta$ with the instanton angle.

One can see that the general solution of the DiracSchwinger-Zwanziger condition (II.8) is

$$
\begin{aligned}
q & =q_{0}\left(n_{e}+\frac{\theta}{2 \pi} n_{m}\right), \\
g & =n_{m} g_{0}
\end{aligned}
$$

with $n_{e}$ and $n_{m}$ integer numbers These equations can be expressed in terms of the complex number

$$
q+i g=q_{0}\left(n_{e}+n_{m} \tau\right)
$$

where

$$
\tau \equiv \frac{\theta}{2 \pi}+\frac{2 \pi i m_{0}}{q_{0}^{2}}
$$

Observe that this definition only includes intrinsic parameters of the theory, and that the imaginary part of $\tau$ is positive definite. This complex parameter will play an important role in supersymmetric gauge theories. Thus, physical states with electric and magnetic charges $(q, g)$ are located on a discrete two dimensional lattice with periods $q_{0}$ and $q_{0} \tau$, and are represented by the corresponding vector $\left(n_{m}, n_{e}\right)$ (see fig. 11). 


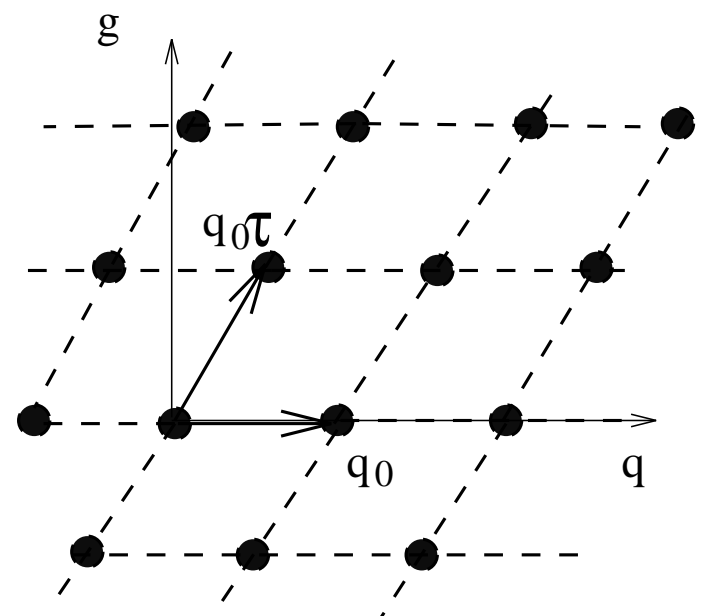

FIG. 1. The charge lattice with periods $q_{0}$ and $q_{0} \tau$. The physical states are located on the points of the lattice.

Notice that the lattice of charges obtained from the quantization condition breaks the classical duality symmetry group $S O(2)$ that rotated the electric and magnetic charges (I.6). But another symmetry group arises at quantum level. Given a lattice as in figure 1 we can describe it in terms of different fundamental cells. Different choices correspond to transforming the electric and magnetic numbers $\left(n_{m}, n_{e}\right)$ by a two-by-two matrix:

$$
\left(n_{m}, n_{e}\right) \rightarrow\left(n_{m}, n_{e}\right)\left(\begin{array}{ll}
\alpha & \beta \\
\gamma & \delta
\end{array}\right)^{-1}
$$

with $\alpha, \beta, \gamma, \delta \in \mathbf{Z}$ satisfying $\alpha \delta-\beta \gamma=1$. This transformation leaves invariant the Dirac-Schwinger-Zwanziger quantiztion condition (II.8). Hence the duality transformations are elements of the discrete group $S L(2, \mathbf{Z})$. Its action on the charge lattice can be implemented by modular transformations of the parameter $\tau$

$$
\tau \rightarrow \frac{\alpha \tau+\beta}{\gamma \tau+\delta} .
$$

This transformations preserve the sign of the imaginary part of $\tau$, and are generated just by the action of two elements:

$$
\begin{array}{ll}
T: & \tau \rightarrow \tau+1, \\
S: & \tau \rightarrow \frac{-1}{\tau} .
\end{array}
$$

The effect of $T$ is to shift $\theta \rightarrow \theta+2 \pi$. Its action is well understood: it just maps the charge lattice $\left(n_{m}, n_{e}\right)$ to $\left(n_{m}, n_{e}-n_{m}\right)$. As physics is $2 \pi$-periodic in $\theta$, it is a symmetry of the theory. Then, if the state $(1,0)$ is in the physical spectrum, the state $\left(1, n_{e}\right)$, with any integer $n_{e}$, is also a physical state.

The effect of $S$ is less trivial. If we take $\theta=0$ just for simplicity, the $S$ action is $q_{0} \rightarrow g_{0}$ and sends the lattice vector $\left(n_{m}, n_{e}\right)$ to the lattice vector $\left(-n_{e}, n_{m}\right)$. So it interchanges the electric and magnetic roles. In terms of coupling constants, it represents the transformation $\tau \rightarrow-1 / \tau$, implying the exchange between the weak and strong coupling regimes. In this respect the duality symmetry could provide a new source of information on nonpertubative physics.

If we claim that the $S$ transformation is also a symmetry of the theory we have full $S L(2, \mathbf{Z})$ symmetry. It implies the existence of any state $\left(n_{m}, n_{e}\right)$ in the physical spectrum, with $n_{m}$ and $n_{e}$ relatively to-prime, just from the knowledge that there are the physical states $\pm(0,1)$ and $\pm(1,0)$. There are some examples of theories 'duality invariant', for instance the $S U(2)$ gauge theory with $N=4$ supersymmetry and the $S U(2)$ gauge theory with $N=2$ supersymmetry and four flavors [4].

A priori however there is no physical reason to impose $S$-invariance, in contrast with $T$-invariance. The stable physical spectrum may not be $S L(2, \mathbf{Z})$ invariant. But if the theory still admits somehow magnetic monopoles, we could apply the $S$-transformation as a change of variables of the theory, where a magnetic state is mapped to an electric state in terms of the dual variables. It could be convenient for several reasons: Maybe there are some physical phenomena where the magnetic monopoles become relevant degrees of freedom; this is the case for the mechanism of confinement, as we will see below. The other reason could be the difficulty in the computation of some dynamical effects in terms of the original electric variables because of the large value of the electric coupling $q_{0}$. The $S$-transformation sends $q_{0}$ to $1 / q_{0}$. In terms of the dual magnetic variables, the physics is weakly coupled.

Just by general arguments we have learned a good deal of information about the duality transformations. Next we have to see where such concepts appear in quantum field theory.

\section{THE HIGGS PHASE}

\section{A. The Higgs mechanism and mass gap.}

We start considering that the relevant degrees of freedom at large distances of some theory in $3+1$ dimensions are reduced to an Abelian Higgs model:

$$
\begin{aligned}
\mathcal{L}\left(\phi^{*}, \phi, A_{\mu}\right) & =-\frac{1}{4} F_{\mu \nu} F^{\mu \nu}+\left(D_{\mu} \phi\right)^{*}\left(D^{\mu} \phi\right) \\
& -\frac{\lambda}{2}\left(\phi^{*} \phi-M^{2}\right)^{2}
\end{aligned}
$$

where

$$
\begin{aligned}
F_{\mu \nu} & =\partial_{\mu} A_{\nu}-\partial_{\nu} A_{\mu}, \\
D_{\mu} \phi & =\left(\partial_{\mu}+i q A_{\mu}\right) \phi,
\end{aligned}
$$

and $q$ is the electric charge of the particle $\phi$. 
An important physical example of a theory described at large distances by the effective Lagrangian (IV.1) (in its nonrelativistic approximation) is a superconductor. Sound waves of a solid material causes complicated deviations from the ideal lattice of the material. Conducting electrons interact with the quantums of those sound waves, called phonons. For electrons near the Fermi surface, their interactions with the phonons create an attractive force. This force can be strong enough to cause bound states of two electrons with opposite spin, called Cooper pairs. The lowest state is a scalar particle with charge $q=-2 e$, which is represented by $\phi$ in ([V.1). To understand the basic features of a superconductor we only need to consider its relevant self-interactions and the interaction with the electromagnetic field resulting from its electric charge $q$. This is the dynamics which is encoded in the effective Lagrangian (IV.1). The values of the parameters $\lambda$ and $M^{2}$ depend of the temperature $T$, and in general contribute to increase the energy of the system. To have an stable ground state, we require $\lambda(T)>0$ for any value of the temperature. But the function $M^{2}(T)$ do not need to be negative for all $T$. In fact, when the temperature $T$ drops below a critical value $T_{c}$, the function $M^{2}(T)$ becomes positive. In such situation, the ground state reaches its minimal energy when the Higgs particle condenses,

$$
|\langle\phi\rangle|=M .
$$

If we make perturbation theory around this minima,

$$
\phi(x)=M+\varphi(x),
$$

with vanishing external electromagnetic fields, we find that there is a mass gap between the ground state and the first excited levels. There are particles of spin one with mass square

$$
\mathcal{M}_{V}^{2}=2 q M^{2},
$$

which corresponds to the inverse of the penetration depth of static electromagnetic fields in the superconductor. There are also spin zero particles with mass square

$$
\mathcal{M}_{H}^{2}=2 \lambda M^{2} .
$$

So perturbation theory already shows a quite different behavior of the Higgs theory from the Coulomb theory. There is only one real massive scalar field and the electromagnetic interaction becomes short-ranged, with the photon correlator being exponentially suppressed. This is a distinction that must survive nonperturbatively. But up to now, the above does not yet distinguish a Higgs theory from just any non-gauge theory with massive vector particles. There is yet another new phenomena in the Higgs mode which shows the spontaneous symmetry breaking of the $U(1)$ gauge theory.

\section{B. Vortex tubes and flux quantization.}

We have seen that the Higgs condensation produces the electromagnetic interactions to be short-range. Ignoring boundary effects in the material, the electric and magnetic fields are zero inside the superconductor. This phenomena is called the Meissner effect.

If we turn on an external magnetic field $\mathbf{H}_{0}$ beyond some critical value, one finds that small regions in the superconductor make a transition to a 'nonsuperconducting' state. Stable magnetic flux tubes are allowed along the material, with a transverse size of the order of the inverse of the mass gap. Their magnetic flux satisfy a quantization rule that can be understood only by a combination of the spontaneous symmetry breaking of the $U(1)$ gauge symmetry and some topological arguments.

Parameterize the complex Higgs field by

$$
\phi(x)=\rho(x) e^{i \chi(x)},
$$

and perform fluctuations around the configuration which minimizes the energy. i.e., we consider that $\rho(x) \simeq M$ almost everywhere, but at some points $\rho$ may be zero. At such points $\chi$ needs not be well defined and therefore in all the rest of space $\chi$ could be multivalued. For instance, if we take a closed contour $C$ around a zero of $\rho(x)$, then following $\chi$ around $C$ could give values that run from 0 to $2 \pi n$, with $n$ an integer number, instead of coming back to zero. These are exactly the field configurations that produce the quantized magnetic flux tubes [5].

Consider a two-dimensional plane, cut somewhere through a superconducting piece of material, with polar coordinates $(r, \theta)$ and work in the time-like $A_{0}=0$. To have a finite energy per unit length static configuration we should demand that

$$
\begin{gathered}
\phi(x) \rightarrow M e^{i \chi(\theta)}, \\
A_{i}(x) \rightarrow \frac{\mathrm{const}}{r},
\end{gathered}
$$

for $r \rightarrow \infty$. Obviously, to keep the fields single valued, we must have

$$
\chi(2 \pi)=\chi(0)+2 \pi n .
$$

If $n \neq 0$, it is clear that at some point of the twodimensional plane we should have that the continuous field $\phi$ vanishes. Such field configurations do not correspond to the ground state.

Solve the field equations with the boundary conditions (IV.8) and (IV.9) fixed, and minimize the energy. We find stable vortex tubes with non-trivial magnetic flux through the two-dimensional plane. To see this, perform a singular gauge transformation $\mathrm{f}$

\footnotetext{
${ }^{\ddagger}$ Singular in the sense of being not well defined in all space.
} 


$$
\begin{array}{r}
\phi(x) \rightarrow e^{i q \Lambda(x)} \phi(x), \\
A_{\mu}(x) \rightarrow A_{\mu}(x)-\partial_{\mu} \Lambda(x),
\end{array}
$$

with $\Lambda=2 \pi n \theta / q$. We compute the magnetic flux in such a gauge and we find

$$
\Phi=\oint A_{\mu} d x^{\mu}=\Lambda(2 \pi)-\Lambda(0)=\frac{2 \pi n}{q} .
$$

It is important to realize that such field configurations, called Abrikosov vortices, are stable. The vortex tube cannot break since it cannot have an end point: as the magnetic flux is quantized, we would have be able to deform continuously the singular gauge transformation $\Lambda$ to zero, something obviously not possible for $n \neq 0$. Physically this is the statement that the magnetic flux is conserved, a consequence of the Maxwell equations. Mathematically it means that for $n \neq 0$ the function $\chi(\theta)$ belongs to a nontrivial homotopy class of the fundamental group $\Pi_{1}(U(1))=\mathbf{Z}$.

The existence of these macroscopic stable objects can be used as another characterization of the Higgs phase. They should survive beyond perturbation theory.

\section{Magnetic monopoles and permanent magnetic confinement.}

The magnetic flux conservation in the Abelian Higgs model tells us that the theory does not include magnetic monopoles. But it is remarkable that the magnetic flux is precisely a multiple of the quantum of magnetic charge $2 \pi / q$ found by Dirac. If we imagine the effective gauge theory (IV.1) enriched somehow by magnetic monopoles, they would form end points of the vortex tubes. The energy per unit length, i.e., the string tension $\sigma$, of these flux tubes is of the order of the scale of the Higgs condensation,

$$
\sigma \sim M^{2}
$$

It implies that the total energy of a system composed of a monopole and an anti-monopole, with a convenient magnetic flux tube attached between them, would be at least proportional to the separation length of the monopoles. In other words: magnetic monopoles in the Higgs phase are permanently confined.

\section{THE GEORGI-GLASHOW MODEL AND THE COULOMB PHASE.}

The Georgi-Glashow model is a Yang-Mills-Higgs system which contains a Higgs multiplet $\phi^{a}(a=1,2,3)$ transforming as a vector in the adjoint representation of the gauge group $S O(3)$, and the gauge fields $W_{\mu}=$ $W_{\mu}^{a} T^{a}$. Here, $T^{a}$ are the hermitian generators of $S O(3)$ satisfying $\left[T^{a}, T^{b}\right]=i f^{a b c} T^{c}$. In the adjoint representation, we have $\left(T^{a}\right)_{b c}=-i f_{b c}^{a}$ and, for $S O(3), f^{a b c}=\epsilon^{a b c}$.
The field strength of $W_{\mu}$ and the covariant derivative on $\phi^{a}$ are defined by

$$
\begin{aligned}
G_{\mu \nu} & =\partial_{\mu} W_{\nu}-\partial_{\nu} W_{\mu}+i e\left[W_{\mu}, W_{\nu}\right], \\
D_{\mu} \phi^{a} & =\partial_{\mu} \phi^{a}-e \epsilon^{a b c} W_{\mu}^{b} \phi^{c} .
\end{aligned}
$$

The minimal Lagrangian is then given by

$$
\begin{aligned}
\mathcal{L} & =-\frac{1}{4} G_{\mu \nu}^{a} G^{a \mu \nu} \\
& +\frac{1}{2} D^{\mu} \phi^{a} D_{\mu} \phi^{a}-V(\phi),
\end{aligned}
$$

where,

$$
V(\phi)=\frac{\lambda}{4}\left(\phi^{a} \phi^{a}-a^{2}\right)^{2} .
$$

The equations of motion following from this Lagrangian are

$$
\begin{aligned}
\left(D_{\nu} G^{\mu \nu}\right)^{a}= & -e \epsilon^{a b c} \phi^{b}\left(D^{\mu} \phi\right)^{c}, \\
D^{\mu} D_{\mu} \phi^{a} & =-\lambda \phi^{a}\left(\phi^{2}-a^{2}\right) .
\end{aligned}
$$

The gauge field strength also satisfies the Bianchi identity

$$
D_{\nu} \widetilde{G}^{\mu \nu a}=0 .
$$

Let us find the vacuum configurations in this theory. Introducing non-Abelian electric and magnetic fields, $G_{a}^{0 i}=-\mathcal{E}_{a}^{i}$ and $G_{a}^{i j}=-\epsilon^{i j}{ }_{k} \mathcal{B}_{a}^{k}$, the energy density is written as

$$
\begin{aligned}
\theta_{00} & =\frac{1}{2}\left(\left(\mathcal{E}_{a}^{i}\right)^{2}+\left(\mathcal{B}_{a}^{i}\right)^{2}\right. \\
& \left.+\left(D^{0} \phi_{a}\right)^{2}+\left(D^{i} \phi_{a}\right)^{2}\right)+V(\phi) .
\end{aligned}
$$

Note that $\theta_{00} \geq 0$, and it vanishes only if

$$
G_{a}^{\mu \nu}=0, \quad D_{\mu} \phi=0, \quad V(\phi)=0 .
$$

The first equation implies that in the vacuum, $W_{\mu}^{a}$ is pure gauge and the last two equations define the Higgs vacuum. The structure of the space of vacua is determined by $V(\phi)=0$ which solves to $\phi^{a}=\phi_{v a c}^{a}$ such that $\left|\phi_{v a c}\right|=a$. The space of Higgs vacua is therefore a twosphere $\left(S^{2}\right)$ of radius $a$ in field space. To formulate a perturbation theory, we have to choose one of these vacua and hence, break the gauge symmetry spontaneously The part of the symmetry which keeps this vacuum invariant, still survives and the corresponding unbroken generator is $\phi_{v a c}^{c} T^{c} / a$. The gauge boson associated with this generator is $A_{\mu}=\phi_{v a c}^{c} W_{\mu}^{c} / a$ and the electric charge operator for this surviving $U(1)$ is given by

$$
Q=e \frac{\phi_{v a c}^{c} T^{c}}{a}
$$

If the group is compact, this charge is quantized. The perturbative spectrum of the theory can be found by expanding $\phi^{a}$ around the chosen vacuum as

$$
\phi^{a}=\phi_{v a c}^{a}+\phi^{\prime a} .
$$


A convenient choice is $\phi_{v a c}^{c}=\delta^{c 3} a$. The perturbative spectrum (which becomes manifest after choosing an appropriate unitary gauge) consists of a massive Higgs of spin zero with a square mass

$$
\mathcal{M}_{H}^{2}=2 \lambda a^{2},
$$

a massless photon, corresponding to the $U(1)$ gauge boson $A_{\mu}^{3}$, and two charged massive W-bosons, $A_{\mu}^{1}$ and $A_{\mu}^{2}$, with square mass

$$
\mathcal{M}_{W}^{2}=e^{2} a^{2} .
$$

This mass spectrum is realistic as long as we are at weak coupling, $e^{2} \sim \lambda \ll 1$. At strong coupling, nonperturbative effects could change significatively eqs. (V.9) and $(\mathrm{V} .10)$. But the fact that there is an unbroken subgroup of the gauge symmetry ensures that there is some massless gauge boson, which a long range interaction. This is the characteristic of the Coulomb phase.

\section{THE' 'T HOOFT-POLYAKOV MONOPOLES}

Let us look for time-independent, finite energy solutions in the Georgi-Glashow model. Finiteness of energy requires that as $r \rightarrow \infty$, the energy density $\theta_{00}$ given by (V.6) must approach zero faster than $1 / r^{3}$. This means that as $r \rightarrow \infty$, our solution must go over to a Higgs vacuum defined by (V.7). In the following, we will first assume that such a finite energy solution exists and show that it can have a monopole charge related to its soliton number which is, in turn, determined by the associated Higgs vacuum. This result is proven without having to deal with any particular solution explicitly. Next, we will describe the 't Hooft-Polyakov ansatz for explicitly constructing one such monopole solution, where we will also comment on the existence of Dyonic solutions. In the last two subsections we will derive the Bogomol'nyi bound and the Witten effect.

\section{A. The Topological nature of the magnetic charge.}

For convenience, in this subsection we will use the vector notation for the $S O(3)$ gauge group indices and not for the spatial indices.

Let $\vec{\phi}_{v a c}$ denote the field $\vec{\phi}$ in a Higgs vacuum. It then satisfies the equations

$$
\begin{gathered}
\vec{\phi}_{v a c} \cdot \vec{\phi}_{v a c}=a^{2}, \\
\partial_{\mu} \vec{\phi}_{v a c}-e \vec{W}_{\mu} \times \vec{\phi}_{v a c}=0,
\end{gathered}
$$

which can be solved for $\vec{W}_{\mu}$. The most general solution is given by

$$
\vec{W}_{\mu}=\frac{1}{e a^{2}} \vec{\phi}_{v a c} \times \partial_{\mu} \vec{\phi}_{v a c}+\frac{1}{a} \vec{\phi}_{v a c} A_{\mu} .
$$

To see that this actually solves VI.1, note that $\partial_{\mu} \vec{\phi}_{v a c}$. $\vec{\phi}_{v a c}=0$, so that

$$
\begin{aligned}
& \frac{1}{e a^{2}}\left(\vec{\phi}_{v a c} \times \partial_{\mu} \vec{\phi}_{v a c}\right) \times \vec{\phi}_{v a c}= \\
& \frac{1}{e a^{2}}\left(\partial_{\mu} \vec{\phi}_{v a c} a^{2}-\vec{\phi}_{v a c}\left(\vec{\phi}_{v a c} \cdot \partial_{\mu} \phi_{v a c}\right)\right)=\frac{1}{e} \partial_{\mu} \vec{\phi}_{v a c} .
\end{aligned}
$$

The first term on the right-hand side of Eq. (VI.2) is the particular solution, and $\vec{\phi}_{v a c} A_{\mu}$ is the general solution to the homogeneous equation. Using this solution, we can now compute the field strength tensor $\vec{G}_{\mu \nu}$. The field strength $F_{\mu \nu}$ corresponding to the unbroken part of the gauge group can be identified as

$$
\begin{aligned}
& F_{\mu \nu}=\frac{1}{a} \vec{\phi}_{v a c} \cdot \vec{G}_{\mu \nu}=\partial_{\mu} A_{\nu}-\partial_{\nu} A_{\mu} \\
& +\frac{1}{a^{3} e} \vec{\phi}_{v a c} \cdot\left(\partial_{\mu} \vec{\phi}_{v a c} \times \partial_{\nu} \vec{\phi}_{v a c}\right) .
\end{aligned}
$$

Using the equations of motion in the Higgs vacuum it follows that

$$
\partial_{\mu} F^{\mu \nu}=0, \quad \partial_{\mu} \widetilde{F}^{\mu \nu}=0 .
$$

This confirms that $F_{\mu \nu}$ is a valid $U(1)$ field strength tensor. The magnetic field is given by $B^{i}=-\frac{1}{2} \epsilon^{i j k} F_{j k}$. Let us now consider a static, finite energy solution and a surface $\Sigma$ enclosing the core of the solution. We take $\Sigma$ to be far enough so that, on it, the solution is already in the Higgs vacuum. We can now use the magnetic field in the Higgs vacuum to calculate the magnetic charge $g_{\Sigma}$ associated with our solution:

$$
\begin{aligned}
& g_{\Sigma}=\int_{\Sigma} B^{i} d s^{i} \\
& =-\frac{1}{2 e a^{3}} \int_{\Sigma} \epsilon_{i j k} \vec{\phi}_{v a c} \cdot\left(\partial^{j} \vec{\phi}_{v a c} \times \partial^{k} \vec{\phi}_{v a c}\right) d s^{i} .
\end{aligned}
$$

It turns out that the expression on the right hand side is a topological quantity as we explain below: Since $\phi^{2}=a$; the manifold of Higgs vacua $\left(\mathcal{M}_{0}\right)$ has the topology of $S^{2}$. The field $\vec{\phi}_{v a c}$ defines a map from $\Sigma$ into $\mathcal{M}_{0}$. Since $\Sigma$ is also an $S^{2}$, the map $\phi_{v a c}: \Sigma \rightarrow \mathcal{M}_{0}$ is characterized by its homotopy group $\pi_{2}\left(S^{2}\right)$. In other words, $\phi_{v a c}$ is characterized by an integer $\nu$ (the winding number) which counts the number of times it wraps $\Sigma$ around $\mathcal{M}_{0}$. In terms of the map $\phi_{v a c}$, this integer is given by

$$
\nu=\frac{1}{4 \pi a^{3}} \int_{\Sigma} \frac{1}{2} \epsilon_{i j k} \vec{\phi}_{v a c} \cdot\left(\partial^{j} \vec{\phi}_{v a c} \times \partial^{k} \vec{\phi}_{v a c}\right) d s^{i} .
$$

Comparing this with the expression for magnetic charge, we get the important result

$$
g_{\Sigma}=\frac{-4 \pi \nu}{e} .
$$

Hence, the winding number of the soliton determines its monopole charge. Note that the above equation differs 
from the Dirac quantization condition by a factor of 2 . This is because the smallest electric charge which could exist in our model is $e / 2$ for an spinorial representation of $S U(2)$, the universal covering group of $S O(3)$. Then, in this model $m_{0}=2$.

\section{B. The 't Hooft-Polyakov ansatz.}

Now we describe an ansatz proposed by 't Hooft [6] and Polyakov [7] for constructing a monopole solution in the Georgi-Glashow model. For a spherically symmetric, parity-invariant, static solution of finite energy, they proposed:

$$
\begin{aligned}
\phi^{a} & =\frac{x^{a}}{e r^{2}} H(\text { aer }), \\
W_{i}^{a} & =-\epsilon_{i j}^{a} \frac{x^{j}}{e r^{2}}(1-K(\text { aer })), \\
W_{0}^{a} & =0 .
\end{aligned}
$$

For the non-trivial Higgs vacuum at $r \rightarrow \infty$, they chose $\phi_{v a c}^{c}=a x^{c} / r=a \hat{x}^{c}$. Note that this maps an $S^{2}$ at spatial infinity onto the vacuum manifold with a unit winding number. The asymptotic behavior of the functions $H(a e r)$ and $K($ aer $)$ are determined by the Higgs vacuum as $r \rightarrow \infty$ and regularity at $r=0$. Explicitly, defining $\xi=a e r$, we have: as $\xi \rightarrow \infty, H \sim \xi, K \rightarrow 0$ and as $\xi \rightarrow 0, H \sim \xi,(K-1) \sim \xi$. The mass of this solution can be parameterized as

$$
\mathcal{M}=\frac{4 \pi a}{e} f\left(\lambda / e^{2}\right) .
$$

For this ansatz, the equations of motion reduce to two coupled equations for $K$ and $H$ which have been solved exactly only in certain limits. For $r \rightarrow 0$, one gets $H \rightarrow e c_{1} r^{2}$ and $K=1+e c_{2} r^{2}$ which shows that the fields are non-singular at $r=0$. For $r \rightarrow \infty$, we get $H \rightarrow \xi+c_{3} \exp (-a \sqrt{2 \lambda} r)$ and $K \rightarrow c_{4} \xi \exp (-\xi)$ which leads to $W_{i}^{a} \approx-\epsilon_{i j}^{a} x^{j} / e r^{2}$. Once again, defining $F_{i j}=\phi^{c} G_{i j}^{c} / a$, the magnetic field turns out to be $B^{i}=-x^{i} / e r^{3}$. The associated monopole charge is $g=-4 \pi / e$, as expected from the unit winding number of the solution. It should be mentioned that 't Hooft's definition of the Abelian field strength tensor is slightly different but, at large distances, it reduces to the form given above.

Note that in the above monopole solution, the presence of the Dirac string is not obvious. To extract the Dirac string, we have to perform a singular gauge transformation on this solution which rotates the non-trivial Higgs vacuum $\phi_{v a c}^{c}=a \hat{x}^{c}$ into the trivial vacuum $\phi_{v a c}^{c}=a \delta^{c 3}$. In the process, the gauge field develops a Dirac string singularity which now serves as the source of the magnetic charge [6].

The 't Hooft-Polyakov monopole carries one unit of magnetic charge and no electric charge. The GeorgiGlashow model also admits solutions which carry both magnetic as well as electric charges. An ansatz for constructing such a solution was proposed by Julia and Zee [8]. In this ansatz, $\phi^{a}$ and $W_{i}^{a}$ have exactly the same form as in the 't Hooft-Polyakov ansatz, but $W_{0}^{a}$ is no longer zero: $W_{0}^{a}=x^{a} J($ aer $) / e r^{2}$. This serves as the source for the electric charge of the dyon. It turns out that the dyon electric charge depends of a continuous parameter and, at the classical level, does not satisfy the quantization condition. However, semiclassical arguments show that, in $\mathrm{CP}$ invariant theories, and at the quantum level, the dyon electric charge is quantized as $q=n e$. This can be easily understood if we recognize that a monopole is not invariant under a gauge transformation which is, of course, a symmetry of the equations of motion. To deal with the associated zero-mode properly, the gauge degree of freedom should be regarded as a collective coordinate. Upon quantization, this collective coordinate leads to the existence of electrically charged states for the monopole with discrete charges. In the presence of a $\mathrm{CP}$ violating term in the Lagrangian, the situation is more subtle as we will discuss later. In the next subsection, we describe a limit in which the equations of motion can be solved exactly for the 'tHooft-Polyakov and the Julia-Zee ansatz. This is the limit in which the soliton mass saturates the Bogomol'nyi bound.

\section{The Bogomol'nyi bound and the BPS states.}

In this subsection, we derive the Bogomol'nyi bound [9] on the mass of a dyon in term of its electric and magnetic charges which are the sources for $F^{\mu \nu}=\vec{\phi} \cdot \vec{G}^{\mu \nu} / a$. Using the Bianchi identity (V.5) and the first equation in (V.4), we can write the charges as

$$
\begin{aligned}
g & \equiv \int_{S_{\infty}^{2}} B_{i} d S^{i}=\frac{1}{a} \int \mathcal{B}_{i}^{a}\left(D^{i} \phi\right)^{a} d^{3} x, \\
q & \equiv \int_{S_{\infty}^{2}} E_{i} d S^{i}=\frac{1}{a} \int \mathcal{E}_{i}^{a}\left(D^{i} \phi\right)^{a} d^{3} x .
\end{aligned}
$$

Now, in the center of mass frame, the dyon mass is given by

$$
\begin{array}{r}
\mathcal{M} \equiv \int d^{3} x \theta_{00}=\int d^{3} x\left(\frac { 1 } { 2 } \left[\left(\mathcal{E}_{k}^{a}\right)^{2}+\left(\mathcal{B}_{k}^{a}\right)^{2}\right.\right. \\
\left.\left.+\left(D_{k} \phi^{a}\right)^{2}+\left(D_{0} \phi^{a}\right)^{2}\right]+V(\phi)\right),
\end{array}
$$

where, $\theta_{\mu \nu}$ is the energy momentum tensor. Using (VI.9) and some algebra we obtain

$$
\begin{aligned}
\mathcal{M} & =\int d^{3} x\left(\frac { 1 } { 2 } \left[\left(\mathcal{E}_{k}^{a}-D_{k} \phi^{a} \sin \theta\right)^{2}\right.\right. \\
& \left.+\left(\mathcal{B}_{k}^{a}-D_{k} \phi^{a} \cos \theta\right)^{2}+\left(D_{0} \phi^{a}\right)^{2}\right] \\
& +V(\phi))+a(q \sin \theta+g \cos \theta),
\end{aligned}
$$

where $\theta$ is an arbitrary angle. Since the terms in the first line are positive, we can write $\mathcal{M} \geq(q \sin \theta+g \cos \theta)$. 
This bound is maximized for $\tan \theta=q / g$. Thus we get the Bogomol'nyi bound on the dyon mass as

$$
\mathcal{M} \geq a \sqrt{g^{2}+q^{2}} .
$$

For the 't Hooft-Polyakov solution, we have $q=0$, and thus, $\mathcal{M} \geq a|g|$. But $|g|=4 \pi / e$ and $\mathcal{M}_{W}=a e=a q$, so that

$$
\mathcal{M} \geq a \frac{4 \pi}{e}=\frac{4 \pi}{e^{2}} \mathcal{M}_{W}=\frac{4 \pi}{q^{2}} \mathcal{M}_{W}=\frac{\nu}{\alpha} \mathcal{M}_{W} .
$$

Here, $\alpha$ is the fine structure constant and $\nu=1$ or $1 / 4$, depending on whether the electron charge is $q$ or $q / 2$. Since $\alpha$ is a small ( $\sim 1 / 137$ for electromagnetism), the above relation implies that the monopole is much heavier than the W-bosons associated with the symmetry breaking.

From (VI.11) it is clear that the bound is not saturated unless $\lambda \rightarrow 0$, so that $V(\phi)=0$. This is the Bogomol'nyi-Prasad-Sommerfield (BPS) limit of the theory [9, 10]. Note that in this limit, $\phi_{v a c}^{2}=a^{2}$ is no longer determined by the theory and, therefore, has to be imposed as a boundary condition on the Higgs field. Moreover, in this limit, the Higgs scalar becomes massless. Now, to saturate the bound we set

$$
\begin{aligned}
& D_{0} \phi^{a}=0, \\
& \mathcal{E}_{k}^{a}=\left(D_{k} \phi\right)^{a} \sin \theta, \\
& \mathcal{B}_{k}^{a}=\left(D_{k} \phi\right)^{a} \cos \theta,
\end{aligned}
$$

where, $\tan \theta=q / g$. In the BPS limit, one can use the 't Hooft-Polyakov (or the Julia-Zee) ansatz either in (V.4), or in (VI.13) to obtain the exact monopole (or dyon) solutions [9,10]. These solutions automatically saturate the Bogomol'nyi bound and are referred to as the BPS states. Also, note that in the BPS limit, all the perturbative excitations of the theory saturate this bound and, therefore, belong to the BPS spectrum. As we will see later, BPS states appears in a very natural way in theories with $N=2$ supersymmetry.

\section{The $\theta$ parameter and the Witten effect.}

In this section we will show that in the presence of a $\theta$ term in the Lagrangian, the magnetic charge of a particle always contributes to its electric charge in the way given by formula (III.2) 111.

To study the effect of $\mathrm{CP}$ violation, we consider the Georgi-Glashow model with an additional $\theta$-term as the only source of $\mathrm{CP}$ violation:

$$
\begin{aligned}
\mathcal{L} & =-\frac{1}{4} F_{\mu \nu}^{a} F^{a \mu \nu}+\frac{1}{2}\left(D_{\mu} \phi^{a}\right)^{2}-\lambda\left(\phi^{2}-a^{2}\right)^{2} \\
& +\frac{\theta e^{2}}{32 \pi^{2}} F_{\mu \nu}^{a} \tilde{F}^{a \mu \nu} .
\end{aligned}
$$

Here, $\tilde{F}^{a \mu \nu}=\frac{1}{2} \epsilon^{\mu \nu \rho \sigma} F_{\rho \sigma}^{a}$. The presence of the $\theta$-term does not affect the equations of motion but changes the physics since the theory is no longer $\mathrm{CP}$ invariant. We want to construct the electric charge operator in this theory. The theory has an $S O(3)$ gauge symmetry but the electric charge is associated with an unbroken $U(1)$ which keeps the Higgs vacuum invariant. Hence, we define an operator $N$ which implements a gauge rotation around the $\hat{\phi}$ direction with gauge parameter $\Lambda^{a}=\phi^{a} / a$. These transformations correspond to the electric charge. Under $N$, a vector $v^{a}$ and the gauge fields $A_{\mu}^{a}$ transform as

$$
\delta v^{a}=\frac{1}{a} \epsilon^{a b c} \phi^{b} v^{c}, \quad \delta A_{\mu}^{a}=\frac{1}{e a} D_{\mu} \phi^{a} .
$$

Clearly, $\phi^{a}$ is kept invariant. At large distances where $|\phi|=a$, the operator $e^{2 \pi i N}$ is a $2 \pi$-rotation about $\hat{\phi}$ and therefore $\exp (2 \pi i N)=1$. Elsewhere, the rotation angle is $2 \pi|\phi| / a$. However, by Gauss' law, if the gauge transformation is 1 at $\infty$, it leaves the physical states invariant. Thus, it is only the large distance behavior of the transformation which matters and the eigenvalues of $N$ are quantized in integer units. Now, we use Noether's formula to compute $N$ :

$$
N=\int d^{3} x\left(\frac{\delta \mathcal{L}}{\delta \partial_{0} A_{i}^{a}} \delta A_{i}^{a}+\frac{\delta \mathcal{L}}{\delta \partial_{0} \phi^{a}} \delta \phi^{a}\right) .
$$

Since $\delta \vec{\phi}=0$, only the gauge part (which also includes the $\theta$-term) contributes:

$$
\begin{aligned}
& \frac{\delta}{\delta \partial_{0} A_{i}^{a}}\left(F_{\mu \nu}^{a} F^{a \mu \nu}\right)=4 F^{a o i}=-4 \mathcal{E}^{a i}, \\
& \frac{\delta}{\delta \partial_{0} A_{i}^{a}}\left(\tilde{F}_{\mu \nu}^{a} F^{a \mu \nu}\right)=2 \epsilon^{i j k} F_{j k}^{a}=-4 \mathcal{B}^{a i} .
\end{aligned}
$$

Thus,

$$
\begin{aligned}
N & =\frac{1}{a e} \int d^{3} x D_{i} \vec{\phi} \cdot \overrightarrow{\mathcal{E}}^{i}-\frac{\theta e}{8 \pi^{2} a} \int d^{3} x D_{i} \vec{\phi} \cdot \overrightarrow{\mathcal{B}}^{i} \\
& =\frac{1}{e} Q_{e}-\frac{\theta e}{8 \pi^{2}} Q_{m}
\end{aligned}
$$

where, we have used (VI.9). Here, $Q_{e}$ and $Q_{m}$ are the electric and magnetic charge operators with eigenvalues $q$ and $g$, respectively, and $N$ is quantized in integer units. This leads to the following formula for the electric charge:

$$
q=n e+\frac{\theta e^{2}}{8 \pi^{2}} g .
$$

For the 't Hooft-Polyakov monopole, $n=1, g=-4 \pi / e$, and therefore, $q=e(1-\theta / 2 \pi)$. For a general dyonic solution we get

$$
g=\frac{4 \pi}{e} n_{m}, \quad q=n_{e} e+\frac{\theta e}{2 \pi} n_{m} .
$$

and we recover (III.2) and (III.3) for $q_{0}=e$. In the presence of a $\theta$-term, a magnetic monopole always carries an electric charge which is not an integral multiple of 
some basic unit. In section III we introduced the charge lattice of periods $e$ and $e \tau$. In this parameterization, the Bogomol'nyi bound (VI.12) takes the form

$$
\mathcal{M} \geq \sqrt{2}\left|a e\left(n_{e}+n_{m} \tau\right)\right| .
$$

Notice that for a BPS state, equation (VI.16) implies that its mass is proportional to the distance of its lattice point from the origin.

\section{THE CONFINING PHASE.}

\section{A. The Abelian projection.}

In non-Abelian gauge theories, gauge fixing is a subject full of interesting surprises (ghosts, phantom solitons, ...) which often obscure the physical content of the theory [12].

't Hooft gave a qualitative program to overcome these difficulties and provided a scenario that explains confinement in a gauge theory. The idea is to perform the gauge fixing procedure in two steps. In the first one a unitary gauge is chosen for the non-Abelian degrees of freedom. It reduces the non-Abelian gauge symmetry to the maximal Abelian subgroup of the gauge group. Here one gets particle gauge singularities 5 . This procedure is called the Abelian projection [12]. In this way, the dynamics of the Yang-Mils theory will be reduced to an Abelian gauge theory with certain additional degrees of freedom.

We need a field that transforms without derivatives under gauge transformations. An example is a real field, $X$ in the adjoint representation of $S U(N)$,

$$
X \rightarrow \Omega X \Omega^{-1} .
$$

Such a field can always be found; take for instance $X^{a}=G_{12}^{a}$. We will use the field $X$ to implement the unitary gauge condition which will carry us to the Abelian projection of the $S U(N)$ gauge group. The gauge is fixed by requiring that $X$ be diagonal:

$$
X=\left(\begin{array}{ccc}
\lambda_{1} & & 0 \\
& \ddots & \\
0 & & \lambda_{N}
\end{array}\right)
$$

The eigenvalues of the matrix $X$ are gauge invariant. Generically they are all different, and the gauge condition (VII.2) leaves an Abelian $U(1)^{N-1}$ gauge symmetry. It corresponds to the subgroup generated by the gauge transformations

$$
\Omega=\left(\begin{array}{ccc}
e^{i \omega_{1}} & & 0 \\
& \ddots & \\
0 & & e^{i \omega_{N}}
\end{array}\right), \quad \sum_{i=1}^{N} \omega_{i}=0 .
$$

\footnotetext{
${ }^{\S}$ We will discuss the physical meaning of them later on.
}

There is also a discrete subgroup of transformations which still leave $X$ in diagonal form. It is the Weyl group of $S U(N)$, which corresponds to permutations of the eigenvalues $\lambda_{i}$. We also fix the Weyl group with the convention $\lambda_{1}>\lambda_{2}>\cdots \lambda_{N}$.

At this stage, we have an Abelian $U(1)$ gauge theory with $N-1$ photons, $N(N-1)$ charged vector particles and some additional degrees of freedom that will appear presently.

\section{B. The nature of the gauge singularities.}

So far we assumed that the eigenvalues $\lambda_{i}$ coincide nowhere. But there are some gauge field configurations that produce two consecutive eigenvalues to coincide at some spacetime points

$$
\lambda_{i}=\lambda_{i+1}=\lambda, \quad \text { for certain } i .
$$

These spacetime points are 'singular' points of the Abelian projection. The $S U(2)$ gauge subgroup corresponding to the $2 \times 2$ block matrix with coinciding eigenvalues leaves invariant the gauge-fixing condition (VII.2).

Let us consider the vicinity of such a point. Prior to the complete gauge-fixing we may take $X$ to be

$$
X=\left(\begin{array}{cccc}
D_{1} & 0 & 0 & 0 \\
0 & \lambda+\epsilon_{3} & \epsilon_{1}-i \epsilon_{2} & 0 \\
0 & \epsilon_{1}+i \epsilon_{2} & \lambda-\epsilon_{3} & 0 \\
0 & 0 & 0 & D_{2}
\end{array}\right),
$$

where $D_{1}$ and $D_{2}$ may safely be considered to be diagonalized because the other eigenvalues do not coincide. With respect to that $S U(2)$ subgroup of $S U(N)$ that corresponds to rotations among the $i$ th and $i+1$ st components, the three fields $\epsilon_{a}(x)$ form an isovector. We may write the central block as

$$
\lambda I_{2}+\epsilon_{a} \sigma^{a},
$$

where $\sigma^{a}$ are the Pauli matrices.

Consider static field configurations. The points of space where the two eigenvalues coincide correspond to the points $\mathbf{x}_{0}$ that satisfy

$$
\epsilon^{a}\left(\mathbf{x}_{0}\right)=0 .
$$

These three equations define a single space point, and then the singularity is particle-like. Which is its physical interpretation?.

By analyticity we have that $\epsilon^{a} \sim\left(x-x_{0}\right)^{a}$, and our gauge condition corresponds to rotating the isovector $\epsilon^{a}$ such that

$$
\epsilon=\left(\begin{array}{c}
0 \\
0 \\
\left|\epsilon_{3}\right|
\end{array}\right) .
$$

From the previous sections, we know that the zero-point of $\epsilon^{a}$ at $\mathbf{x}_{0}$ behaves as a magnetic charge with respect 
to the remaining $U(1) \subset S U(2)$ rotations. We realize that those gauge field configurations that produce such a gauge 'singularities' correspond to magnetic monopoles.

The non-Abelian $S U(N)$ gauge theory is topologically such that it can be cast into a $U(1)^{N-1}$ Abelian gauge theory, which will feature not only electrically charged particles but also magnetic monopoles.

\section{The phases of the Yang-Mills vacuum.}

We can now give a qualitative description of the possible phases of the Yang-Mills vacuum. It is only the dynamics which, as a function of the microscopic bare parameters, determines in which phase the Yang-Mills vacuum is actually realized.

Classically, the Yang-Mills Lagrangian is scale invariant. One can write down field configurations with magnetic charge and arbitrarily low energy. But quantum corrections are likely to violate their masslessness. If dynamics simply chooses to give a positive mass to the monopoles, we are in a Higgs or Coulomb phase. We must look for the magnetic vortex tubes to figure out if we are in a Higgs phase. It will be a signal that the ordinary Higgs mechanism has taken place in the Abelian gauge formulation of the Yang-Mills theory. The role of the dynamically generated Higgs field could be done by some scalar composite operator charged respect the $U(1)^{N-1}$ gauge symmetries. There is also the possibility that no Higgs phenomenon occurs at all in the Abelian sector, or that some $U(1)$ gauge symmetries are not spontaneously broken. In this case we are in the Coulomb phase, with some massless photons, or in a mixed Coulomb-Higgs phase.

There is a third possibility however. Maybe the quantum corrections give a formally negative mass squared for the monopole: a magnetically charged object condenses. We apply an 'electric-magnetic dual transformation' to write an effective Lagrangian which encodes the relevant magnetic degrees of freedom in the infrared limit. In such effective Lagrangian, the Higgs mechanism takes place in terms of dual variables. We are in a dual Higgs phase. We have electric flux tubes with finite energy per unit of length. There is a confining potential between electrically charged objects, like quarks.

In 1994, Seiberg and Witten gave a quantitative proof that such dynamical mechanism of color confinement takes place in $N=2$ super-QCD (SQCD) broken to $N=1$ [13], giving a non-trivial realization of 't Hooft scenario. When $N=2 \mathrm{SQCD}$ is softly broken to $N=0$ the same mechanism of confinement persists [14, 15].

\section{Oblique confinement.}

For simplicity let us consider an $S U(2)$ gauge group. We have seen that for a non-zero CP violating parameter $\theta$, the physical electric charge of a particle with electric (resp. magnetic) number $n_{e}$ (resp. $n_{m}$ ) is:

$$
q=\left(n_{e}+\frac{\theta}{2 \pi} n_{m}\right) e
$$

Dyons with large electric charges may have larger selfenergies contributing positively to their mass squared. If the state $\left(n_{e}, n_{m}\right)$ condenses at $\theta \simeq 0$, it is likely that the state $\left(n_{e}-1, n_{m}\right)$ condenses at $\theta \simeq 2 \pi$. It suggests that there is a phase transition around $\theta \simeq \pi$. Such first order phase transitions has been observed in softly broken $N=2$ SQCD to $N=0$ 16.

't Hooft proposed a new condensation mode at $\theta \simeq \pi$ 12. He imagined the possibility that a bound state of the dyons $\left(n_{e}, n_{m}\right)$ and $\left(n_{e}-1, n_{m}\right)$, with zero electric charge at $\theta=\pi$, could be formed. Its smaller electric charge could favor its condensation, leading to what he called an oblique confinement mode. These oblique modes have also been observed in softly broken $N=2$ SQCD with matter 14.15.

\section{THE HIGGS/CONFINING PHASE.}

In the previous section we have characterized the confining phase as the dual of the Higgs phase, i.e., the physical states are gauge singlets made by the electric degrees of freedom bound by stable electric flux tubes. A good gauge invariant order parameter measuring such behavior is the Wilson loop [17]:

$$
W(C)=\operatorname{Tr} \exp \left(i g \oint_{C} d x^{\mu} A_{\mu}\right) .
$$

For $S U(N)$ Yang-Mills in the confining phase, for contours $C$, the Wilson loop obeys the area law,

$$
\langle W(C)\rangle \sim \exp (-\sigma \cdot(\text { area })),
$$

with $\sigma$ the string tension of the electric flux tube.

But dynamical matter fields in the fundamental representation immediately create a problem in identifying the confining phase of the theory through the Wilson loop. The criterion used for confinement in the pure gauge theory, the energy between static sources, no longer works. Even if the energy starts increasing as the sources separate, it eventually becomes favorable to produce a particle-antiparticle pair out of the vacuum. This pair shields the gauge charge of the sources, and the energy stops growing. So even in a theory that 'looks' very confining our signal fails, and the perimeter law replaces (VIII.2),

$$
\langle W(C)\rangle=\sim \exp (-\Lambda \cdot(\text { perimeter }))
$$

If some scalar field is in the fundamental representation of the gauge group, there is no distinction at all between the confinement phases and the Higgs phase. Using the 
scalar field in the fundamental representation one can build gauge invariant interpolating operators for all possible physical states. As the vacuum expectation value of the Higgs field in the fundamental representation continuously changes from large values to smaller ones, the spectrum of all physical states, and all other measurable quantities, changes smoothly [18. There is no gauge invariant operator which can distinguish between the Higgs or confining phases. We are in a Higgs/confining phase.

In supersymmetric gauge theories, it is common to have scalar fields in the fundamental representation of the gauge group, the scalar quarks. In such situation, when the theory is not in the Coulomb phase, we will see that the theory is presented in a Higgs/confining phase. We could take the phase description which is more appropriate for the theory. For instance, if the theory is in the weak coupling region, it is better to realize it in the Higgs phase; if the theory in the strong coupling region, it is better to think it in a confining phase.

\section{SUPERSYMMETRY}

\section{A. The supersymmetry algebra and its massless representations.}

The $N=1$ supersymmetry algebra is written as 19

$$
\begin{aligned}
& \left\{Q_{\alpha}, \bar{Q}_{\dot{\alpha}}\right\}=2 \sigma_{\alpha \dot{\alpha}}^{\mu} P_{\mu} \\
& \left\{Q_{\alpha}, Q_{\beta}\right\}=0,\left\{\bar{Q}_{\dot{\alpha}}, \bar{Q}_{\dot{\beta}}\right\}=0 .
\end{aligned}
$$

Here, $Q$ and $\bar{Q}$ are the supersymmetry generators and transform as spin $1 / 2$ operators, $\alpha, \dot{\alpha}=1,2$. Moreover, the supersymmetry generators commute with the momentum operator $P_{\mu}$ and hence, with $P^{2}$. Therefore, all states in a given representation of the algebra have the same mass. For a theory to be supersymmetric, it is necessary that its particle content form a representation of the above algebra. The irreducible representations of (IX.1) can be obtained using Wigner's method.

For massless states, we can always go to a frame where $P^{\mu}=E(1,0,0,1)$. Then the supersymmetry algebra becomes

$$
\left\{Q_{\alpha}, \bar{Q}_{\dot{\alpha}}\right\}=\left(\begin{array}{rr}
0 & 0 \\
0 & 4 E
\end{array}\right) .
$$

In a unitary theory the norm of a state is always positive. Since $Q_{\alpha}$ and $\bar{Q}_{\dot{\alpha}}$ are conjugate to each other, and $\left\{Q_{1}, \bar{Q}_{\mathrm{i}}\right\}=0$, it follows that $Q_{1} \mid$ phys $>=\bar{Q}_{\mathrm{i}} \mid$ phys $>=0$. As for the other generators, it is convenient to re-scale them as

$$
a=\frac{1}{2 \sqrt{E}} Q_{2}, \quad a^{\dagger}=\frac{1}{2 \sqrt{E}} \bar{Q}_{\dot{2}} .
$$

Then, the supersymmetry algebra takes the form

$$
\left\{a, a^{\dagger}\right\}=1, \quad\{a, a\}=0, \quad\left\{a^{\dagger}, a^{\dagger}\right\}=0 .
$$

This is a Clifford algebra with 2 fermionic generators and has a 2-dimensional representation. From the point of view of the angular momentum algebra, $a$ is a rising operator and $a^{\dagger}$ is a lowering operator for the helicity of massless states. We choose the vacuum such that $J_{3}\left|\Omega_{\lambda}>=\lambda\right| \Omega_{\lambda}>$ and $a \mid \Omega_{\lambda}>=0$. Then

$$
J_{3}\left(a^{\dagger} \mid \Omega_{\lambda}>\right)=\left(\lambda-\frac{1}{2}\right)\left(a^{\dagger} \mid \Omega_{\lambda}>\right) .
$$

The irreducible representations are not necessarily CPT invariant. Therefore, if we want to assign physical states to these representations, we have to supplement them with their CPT conjugates $\mid-\lambda>_{C P T}$. If a representation is CPT self-conjugate, it is left unchanged. Thus, from a Clifford vacuum with helicity $\lambda=1 / 2$ we obtain the $N=1$ supermultiplet:

$$
\left(\begin{array}{cc}
\{\mid 1 / 2>, & \mid-1 / 2>_{C P T} \\
\{\mid 0>, & \left.\mid 0>_{C P T}\right\}
\end{array}\right)
$$

which contains a Weyl spinor $\psi$ and a complex scalar $\phi$. It is called the scalar multiplet.

The other relevant representation of a renormalizable quantum field theory is the vector multiplet. It is constructed from a Clifford vacuum with helicity $\lambda=1$ :

$$
\left.\left(\begin{array}{cc}
\{\mid 1>, & \left.\mid-1>_{C P T}\right\} \\
\{\mid 1 / 2>, & \mid-1 / 2>_{C P T}
\end{array}\right\}\right) .
$$

It contains a vector $A_{\mu}$ and a Weyl spinor $\lambda$.

\section{B. Superspace and superfields.}

To make supersymmetry linearly realized it is convenient to use the superspace formalism and superfields [20]. Superspace is obtained by adding four spinor degrees of freedom $\theta^{\alpha}, \bar{\theta}_{\dot{\alpha}}$ to the spacetime coordinates $x^{\mu}$. Under the supersymmetry transformations implemented by the operator $\xi^{\alpha} Q_{\alpha}+\bar{\xi}_{\dot{\alpha}} \bar{Q}^{\dot{\alpha}}$ with transformation parameters $\xi$ and $\bar{\xi}$, the superspace coordinates transform as

$$
\begin{aligned}
x^{\mu} & \rightarrow x^{\prime \mu}=x^{\mu}+i \theta \sigma^{\mu} \bar{\xi}-i \xi \sigma^{\mu} \bar{\theta}, \\
\theta & \rightarrow \theta^{\prime}=\theta+\xi \\
\bar{\theta} & \rightarrow \bar{\theta}^{\prime}=\bar{\theta}+\bar{\xi} .
\end{aligned}
$$

These transformations can easily be obtained by the following representation of the supercharges acting on $(x, \theta)$ :

$$
\begin{aligned}
& Q_{\alpha}=\frac{\partial}{\partial \theta^{\alpha}}-i \sigma_{\alpha \dot{\alpha}}^{\mu} \bar{\theta}^{\dot{\alpha}} \partial_{\mu}, \\
& \bar{Q}_{\dot{\alpha}}=-\frac{\partial}{\partial \bar{\theta}^{\dot{\alpha}}}+i \theta^{\alpha} \sigma_{\alpha \dot{\alpha}}^{\mu} \partial_{\mu} .
\end{aligned}
$$

These satisfy $\left\{Q_{\alpha}, \bar{Q}_{\dot{\alpha}}\right\}=2 i \sigma_{\alpha \dot{\alpha}}^{\mu} \partial_{\mu}$. Moreover, using the chain rule, it is easy to see that $\partial / \partial x^{\mu}$ is invariant under 
(IX.5) but not $\partial / \partial \theta$ and $\partial / \partial \bar{\theta}$. Therefore, we introduce the super-covariant derivatives

$$
\begin{aligned}
D_{\alpha} & =\frac{\partial}{\partial \theta^{\alpha}}+i \sigma_{\alpha \dot{\alpha}}^{\mu} \partial_{\mu}, \\
\bar{D}_{\dot{\alpha}} & =-\frac{\partial}{\partial \bar{\theta}^{\dot{\alpha}}}-i \sigma_{\alpha \dot{\alpha}}^{\mu} \theta^{\alpha} \partial_{\mu} .
\end{aligned}
$$

They satisfy $\left\{D_{\alpha}, \bar{D}_{\dot{\alpha}}\right\}=-2 i \sigma_{\alpha \dot{\alpha}}^{\mu} \partial_{\mu}$ and anti-commute with $Q$ and $\bar{Q}$.

The quantum fields transform as components of a superfield defined on superspace, $F(x, \theta, \bar{\theta})$. Since the $\theta$ variables are anti-commuting, the Taylor expansion of $F(x, \theta, \bar{\theta})$ in $(\theta, \bar{\theta})$ is finite, indicating that the supersymmetry representations are finite dimensional. The coefficients of the expansion are the component fields.

To have irreducible representations we must impose supersymmetric invariant constraints on the superfields. The scalar multiplet (IX.3) is represented by a chiral scalar superfield, $\Phi$, satisfying the chiral constraint

$$
\bar{D}_{\dot{\alpha}} \Phi=0 .
$$

Note that for $y^{\mu}=x^{\mu}+i \theta \sigma^{\mu} \bar{\theta}$, we have $\bar{D}_{\dot{\alpha}} y^{\mu}=$ $0, \quad \bar{D}_{\dot{\alpha}} \theta^{\beta}=0$. Therefore, any function of $(y, \theta)$ is a chiral superfield. It can be shown that this also is a necessary condition. Hence, any chiral superfield can be expanded as

$$
\Phi(y, \theta)=\phi(y)+\sqrt{2} \theta \psi(y)+\theta \theta F(y) .
$$

Here, $\psi$ and $\phi$ are the fermionic and scalar components respectively and $F$ is an auxiliary field linear and homogeneous. Similarly, an anti-chiral superfield is defined by $D_{\alpha} \Phi^{\dagger}=0$ and can be expanded as

$$
\Phi^{\dagger}\left(y^{\dagger}, \bar{\theta}\right)=\phi^{\dagger}\left(y^{\dagger}\right)+\sqrt{2 \overline{\theta \psi}}\left(y^{\dagger}\right)+\overline{\theta \theta} F^{\dagger}\left(y^{\dagger}\right),
$$

where, $y^{\mu \dagger}=x^{\mu}-i \theta \sigma^{\mu} \bar{\theta}$.

The vector multiplet (IX.4) is represented off-shell by a real scalar superfield

$$
V=V^{\dagger} .
$$

In local quantum field theories, spin one massless particles carry gauge symmetries [21]. These symmetries commute with the supersymmetry transformations. For a vector superfield, many of its component fields can be gauged away using the Abelian gauge transformation $V \rightarrow V+\Lambda+\Lambda^{\dagger}$, where $\Lambda\left(\Lambda^{\dagger}\right)$ are chiral (anti-chiral) superfields. In the Wess-Zumino gauge [19], it becomes

$$
V=-\theta \sigma^{\mu} \bar{\theta} A_{\mu}+i \theta^{2} \overline{\theta \lambda}-i \bar{\theta}^{2} \theta \lambda+\frac{1}{2} \theta^{2} \bar{\theta}^{2} D .
$$

In this gauge, $V^{2}=\frac{1}{2} A_{\mu} A^{\mu} \theta^{2} \bar{\theta}^{2}$ and $V^{3}=0$. The WessZumino gauge breaks supersymmetry, but not the gauge symmetry of the Abelian gauge field $A_{\mu}$. The Abelian superfield gauge field strength is defined by

$$
W_{\alpha}=-\frac{1}{4} \bar{D}^{2} D_{\alpha} V, \quad \bar{W}_{\dot{\alpha}}=-\frac{1}{4} D^{2} \bar{D}_{\dot{\alpha}} V .
$$

It can be verified that $W_{\alpha}$ is a chiral superfield. Since it is gauge invariant, it can be computed in the Wess-Zumino gauge,

$$
\begin{aligned}
W_{\alpha} & =-i \lambda_{\alpha}(y)+\theta_{\alpha} D-\frac{i}{2}\left(\sigma^{\mu} \bar{\sigma}^{\nu} \theta\right)_{\alpha} F_{\mu \nu} \\
& +\theta^{2}\left(\sigma^{\mu} \partial_{\mu} \bar{\lambda}\right)_{\alpha},
\end{aligned}
$$

where, $F_{\mu \nu}=\partial_{\mu} A_{\nu}-\partial_{\nu} A_{\mu}$.

In the non-Abelian case, $V$ belongs to the adjoint representation of the gauge group: $V=V_{A} T^{A}$, where, $T^{A \dagger}=T^{A}$. The gauge transformations are now implemented by

$$
e^{-2 V} \rightarrow e^{-i \Lambda^{\dagger}} e^{-2 V} e^{i \Lambda},
$$

where $\Lambda=\Lambda_{A} T^{A}$ is a chiral superfield. The non-Abelian gauge field strength is defined by

$$
W_{\alpha}=\frac{1}{8} \bar{D}^{2} e^{2 V} D_{\alpha} e^{-2 V}
$$

and transforms as

$$
W_{\alpha} \rightarrow W_{\alpha}^{\prime}=e^{-i \Lambda} W_{\alpha} e^{i \Lambda} .
$$

In components, in the $\mathrm{WZ}$ gauge it takes the form

$$
\begin{aligned}
W_{\alpha}^{a} & =-i \lambda_{\alpha}^{a}+\theta_{\alpha} D^{a}-\frac{i}{2}\left(\sigma^{\mu} \bar{\sigma}^{\nu} \theta\right)_{\alpha} F_{\mu \nu}^{a} \\
& +\theta^{2} \sigma^{\mu} D_{\mu} \bar{\lambda}^{a}
\end{aligned}
$$

where,

$$
\begin{array}{r}
F_{\mu \nu}^{a}=\partial_{\mu} A_{\nu}^{a}-\partial_{\nu} A_{\mu}^{a}+f^{a b c} A_{\mu}^{b} A_{\nu}^{c}, \\
D_{\mu} \bar{\lambda}^{a}=\partial_{\mu} \bar{\lambda}^{a}+f^{a b c} A_{\mu}^{b} \bar{\lambda}^{c} .
\end{array}
$$

Now we are ready to construct supersymmetric Lagrangians in terms of superfields.

\section{Supersymmetric Lagrangians.}

Clearly, any function of superfields is, by itself, a superfield. Under supersymmetry, the superfield transforms as $\delta F=(\xi Q+\overline{\xi Q}) F$, from which the transformation of the component fields can be obtained. Note that the coefficient of the $\theta^{2} \bar{\theta}^{2}$ component is the field component of highest dimension in the multiplet. Then, its variation under supersymmetry is always a total derivative of other components. Thus, ignoring surface terms, the spacetime integral of this component is invariant under supersymmetry. This tells us that a supersymmetric Lagrangian density may be constructed as the highest dimension component of an appropriate superfield.

Let us first consider the product of a chiral and an anti-chiral superfield $\Phi^{\dagger} \Phi$. This is a general superfield and its highest component can be computed using (IX.9) as 


$$
\begin{aligned}
\left.\Phi^{\dagger} \Phi\right|_{\theta^{2} \bar{\theta}^{2}}= & -\frac{1}{4} \phi^{\dagger} \square \phi-\frac{1}{4} \square \phi^{\dagger} \phi+\frac{1}{2} \partial_{\mu} \phi^{\dagger} \partial^{\mu} \phi \\
& -\frac{i}{2} \psi \sigma^{\mu} \partial_{\mu} \bar{\psi}+\frac{i}{2} \partial_{\mu} \psi \sigma^{\mu} \bar{\psi}+F^{\dagger} F .
\end{aligned}
$$

Dropping some total derivatives we get the free field Lagrangian for a massless scalar and a massless fermion with an auxiliary field.

The product of chiral superfields is a chiral superfield. In general, any arbitrary function of chiral superfields is a chiral superfield:

$$
\begin{aligned}
\mathcal{W}\left(\Phi_{i}\right) & =\mathcal{W}\left(\phi_{i}+\sqrt{2} \theta \psi_{i}+\theta \theta F_{i}\right) \\
& =\mathcal{W}\left(\phi_{i}\right)+\frac{\partial \mathcal{W}}{\partial \phi_{i}} \sqrt{2} \theta \psi_{i} \\
& +\theta \theta\left(\frac{\partial \mathcal{W}}{\partial \phi_{i}} F_{i}-\frac{1}{2} \frac{\partial^{2} \mathcal{W}}{\partial \phi_{i} \phi_{j}} \psi_{i} \psi_{j}\right) .
\end{aligned}
$$

$\mathcal{W}$ is referred to as the superpotential. Moreover, the space of the chiral fields $\Phi$ may have a non-trivial metric $g^{i j}$ in which case the scalar kinetic term, for example, takes the form $g^{i j} \partial_{\mu} \phi_{i}^{\dagger} \partial^{\mu} \phi_{j}$, with appropriate modifications for other terms. In such cases, the free field Lagrangian above has to be replaced by a non-linear $\sigma$ model [22]. Thus, the most general $N=1$ supersymmetric Lagrangian for the scalar multiplet is given by

$$
\mathcal{L}=\int d^{4} \theta K\left(\Phi, \Phi^{\dagger}\right)+\int d^{2} \theta \mathcal{W}(\Phi)+\int d^{2} \overline{\theta \mathcal{W}}\left(\Phi^{\dagger}\right) .
$$

Note that the $\theta$-integrals pick up the highest component of the superfield and in our conventions, $\int d^{2} \theta \theta^{2}=1$ and $\int d^{2} \bar{\theta} \bar{\theta}^{2}=1$. In terms of the non-holomorphic function $K\left(\phi, \phi^{\dagger}\right)$, the metric in field space is given by $g^{i j}=\partial^{2} K / \partial \phi_{i} \partial \phi_{j}^{\dagger}$, i.e., the target space for chiral superfields is always a Kähler space. For this reason, the function $K\left(\Phi, \Phi^{\dagger}\right)$ is referred to as the Kähler potential.

Remember that the super-field strength $W_{\alpha}$ is a chiral superfield spinor. Using the normalization $\operatorname{Tr}\left(T^{a} T^{b}\right)=$ $\frac{1}{2} \delta^{a b}$, we have that

$$
\begin{aligned}
\left.\operatorname{Tr}\left(W^{\alpha} W_{\alpha}\right)\right|_{\theta \theta} & =-i \lambda^{a} \sigma^{\mu} D_{\mu} \bar{\lambda}^{a}+\frac{1}{2} D^{a} D^{a} \\
& -\frac{1}{4} F^{a \mu \nu} F_{\mu \nu}^{a}+\frac{i}{8} \epsilon^{\mu \nu \rho \sigma} F_{\mu \nu}^{a} F_{\rho \sigma}^{a} .
\end{aligned}
$$

The first three terms are real and the last one is pure imaginary. It means that we can include the gauge coupling constant and the $\theta$ parameter in the Lagrangian in a compact form

$$
\begin{aligned}
\mathcal{L} & =\frac{1}{4 \pi} \operatorname{Im}\left(\tau \operatorname{Tr} \int d^{2} \theta W^{\alpha} W_{\alpha}\right) \\
& =-\frac{1}{4 g^{2}} F_{\mu \nu}^{a} F^{a \mu \nu}+\frac{\theta}{32 \pi^{2}} F_{\mu \nu}^{a} \tilde{F}^{a \mu \nu} \\
& +\frac{1}{g^{2}}\left(\frac{1}{2} D^{a} D^{a}-i \lambda^{a} \sigma^{\mu} D_{\mu} \bar{\lambda}^{a}\right),
\end{aligned}
$$

where, $\tau=\theta / 2 \pi+4 \pi i / g^{2}$.

We now include matter fields by the introduction of the chiral superfield $\Phi$ in a given representation of the gauge group in which the generators are the matrices $T_{i j}^{a}$. The kinetic energy term $\Phi^{\dagger} \Phi$ is invariant under global gauge transformations $\Phi^{\prime}=e^{-i \Lambda} \Phi$. In the local case, to insure that $\Phi^{\prime}$ remains a chiral superfield, $\Lambda$ has to be a chiral superfield. The supersymmetric gauge invariant kinetic energy term is then given by $\Phi^{\dagger} e^{-2 V} \Phi$. We are now in a position to write down the full $\mathrm{N}=1$ supersymmetric gauge invariant Lagrangian as

$$
\begin{aligned}
\mathcal{L} & =\frac{1}{8 \pi} \operatorname{Im}\left(\tau \operatorname{Tr} \int d^{2} \theta W^{\alpha} W_{\alpha}\right) \\
& +\int d^{2} \theta d^{2} \bar{\theta}\left(\Phi^{\dagger} e^{-2 V} \Phi\right)+\int d^{2} \theta \mathcal{W}+\int d^{2} \bar{\theta} \overline{\mathcal{W}}
\end{aligned}
$$

Note that since each term is separately invariant, the relative normalization between the scalar part and the Yang-Mills part is not fixed by $N=1$ supersymmetry. In fact, under loop effects, by virtue of the perturbative non-renormalization theorem [23], only the term with the complete superspace integral $\int d^{2} \theta d^{2} \bar{\theta}$ gets an overall renormalization factor $Z(\mu, g(\mu))$, with $\mu$ the renormalization scale and $g(\mu)$ the renormalized gauge coupling constant. Observe the unique dependence on $\operatorname{Re}(\tau)$ in $Z$, breaking the holomorphic $\tau$-dependence of the Lagrangian $\mathcal{L}$. But quantities as the superpotential $\mathcal{W}$ are renormalization group invariant under perturbation theory [23] (we will see dynamically generated superpotentials by nonperturbative effects).

In terms of component fields, the Lagrangian (IX.18) becomes

$$
\begin{aligned}
& \mathcal{L}=-\frac{1}{4 g^{2}} F_{\mu \nu}^{a} F^{a \mu \nu}+\frac{\theta}{32 \pi^{2}} F_{\mu \nu}^{a} \widetilde{F}^{a \mu \nu} \\
& -\frac{i}{g^{2}} \lambda^{a} \sigma^{\mu} D_{\mu} \bar{\lambda}^{a}+\frac{1}{2 g^{2}} D^{a} D^{a} \\
& +\left(\partial_{\mu} \phi-i A_{\mu}^{a} T^{a} \phi\right)^{\dagger}\left(\partial^{\mu} \phi-i A^{a \mu} T^{a} \phi\right)-D^{a} \phi^{\dagger} T^{a} \phi \\
& -i \bar{\psi} \bar{\sigma}^{\mu}\left(\partial_{\mu} \psi-i A_{\mu}^{a} T^{a} \psi\right)+F^{\dagger} F \\
& +\left(-i \sqrt{2} \phi^{\dagger} T^{a} \lambda^{a} \psi+\frac{\partial \mathcal{W}}{\partial \phi} F-\frac{1}{2} \frac{\partial^{2} \mathcal{W}}{\partial \phi \partial \phi} \psi \psi+h . c .\right) .
\end{aligned}
$$

Here, $\mathcal{W}$ denotes the scalar component of the superpotential. The auxiliary fields $F$ and $D^{a}$ can be eliminated by using their equations of motion:

$$
\begin{aligned}
F & =\frac{\partial \mathcal{W}}{\partial \phi}, \\
D^{a} & =g^{2}\left(\phi^{\dagger} T^{a} \phi\right) .
\end{aligned}
$$

The terms involving these fields, thus, give rise to the scalar potential

$$
V=|F|^{2}+\frac{1}{2 g^{2}} D^{a} D^{a} .
$$


Using the supersymmetry algebra (IX.1) it is not difficult to see that the hamiltonian $P^{0}=H$ is a positive semidefinite operator, $\langle H\rangle \geq 0$, and that the ground state has zero energy if and only if it is supersymmetric invariant. At the level of local fields, the equation (IX.22) means that the supersymmetric ground state configuration is such that

$$
F=D^{a}=0
$$

\section{R-symmetry.}

The supercharges $Q_{\alpha}$ and $\bar{Q}_{\dot{\alpha}}$ are complex spinors. In the supersymmetry algebra (IX.1) there is a $U(1)$ symmetry associated to the phase of the supercharges:

$$
\begin{aligned}
& Q \rightarrow Q^{\prime}=e^{i \beta} Q \\
& \bar{Q} \rightarrow \bar{Q}^{\prime}=e^{-i \beta} \bar{Q} .
\end{aligned}
$$

This symmetry is called the $R$-symmetry. It plays an important role in the study of supersymmetric gauge theories.

In terms of superspace, the $R$-symmetry is introduced through the superfield generator $(\theta Q+\overline{\theta Q})$. Then, it rotates the phase of the superspace components $\theta$ and $\bar{\theta}$ in the opposite way as $Q$ and $\bar{Q}$. It gives different $R$ charges for the component fields of a superfield. Consider that the chiral superfield $\Phi$ has $R$-charge $n$,

$$
\Phi(x, \theta) \rightarrow \Phi^{\prime}(x, \theta)=e^{i n \beta} \Phi\left(x, e^{-i \beta} \theta\right) .
$$

In terms of its component fields we have that:

$$
\begin{aligned}
& \phi \rightarrow \phi^{\prime}=e^{i n \beta} \phi, \\
& \psi \rightarrow \psi^{\prime}=e^{i(n-1) \beta} \psi, \\
& F \rightarrow F^{\prime}=e^{i(n-2) \beta} F .
\end{aligned}
$$

Since $d^{2}\left(e^{-i \beta} \theta\right)=e^{2 i \beta} d^{2} \theta$, we derive that the superpotential has $R$-charge two,

$$
\mathcal{W}(\Phi) \rightarrow \mathcal{W}\left(\Phi^{\prime}, \theta\right)=e^{2 i \beta} \mathcal{W}\left(\Phi, e^{-i \beta} \theta\right),
$$

and that the Kähler potential is $R$-neutral.

\section{THE USES OF SUPERSYMMETRY.}

\section{A. Flat directions and super-Higgs mechanism}

We have seen that the fields configuration of the supersymmetric ground state are those corresponding to zero energy. To find them we solve (IX.23). Consider a supersymmetric gauge theory with gauge group $G$, and matter superfields $\Phi_{i}$ in the representation $R(f)$ of $G$. The classical equations of motion of the $D^{a}(a=1, \ldots, \operatorname{dim} G)$ auxiliary fields give

$$
D^{a}=\sum_{f} \phi_{f}^{\dagger} T_{f}^{a} \phi_{f} .
$$

The solutions of $D^{a}=0$ usually lead to the concept of flat directions. They play an important role in the analysis of SUSY theories. These flat directions may be lifted by $F$-terms in the Lagrangian, as for instance mass terms.

As an illustrative example of flat directions and some of its consequences, consider the $S U(2)$ gauge group, one chiral superfield $Q$ in the fundamental representation of $S U(2)$ and another chiral superfield $\tilde{Q}$ in the anti-fundamental representation of $S U(2)$. This is supersymmetric QCD (SQCD) with one massless flavor. In this particular case, the equation (X.1) becomes

$$
D^{a}=q^{\dagger} \sigma^{a} q-\tilde{q} \sigma^{a} \tilde{q}^{\dagger} .
$$

The equations $D^{a}=0$ have the general solution (up to gauge and global symmetry transformations)

$$
q=\tilde{q}^{\dagger}=\left(\begin{array}{l}
a \\
0
\end{array}\right), \quad a \text { arbitrary } .
$$

The scalar superpartners of the fermionic quarks, $(q, q)$, called squarks, play the role of Higgs fields. As these are in the fundamental representation of the gauge group, $S U(2)$ is completely broken by the super-Higgs mechanism (for $a \neq 0$ ). It is just the supersymmetric generalization of the familiar Higgs mechanism: three real scalars are eaten by the gluon, in the adjoint representation, and three Weyl spinor combinations of the quark spinors are eaten by the gluino to form a massive Dirac spinor in the adjoint of $S U(2)$. Gluons and gluinos acquire the classical square mass

$$
\mathcal{M}_{g}^{2}=2 g_{0}^{2}|a|^{2}
$$

where $g_{0}$ is the bare gauge coupling. We see that the theory is in the Higgs/confining phase. But there is not mass gap; it remains a massless superfield. Its corresponding massless scalar must move along some flat direction of the classical potential. This flat direction is given by the arbitrary value of the real number $|a|$. This degeneracy is not unphysical, as in the spontaneous breaking of a symmetry. When we move along the supersymmetric flat direction the physical observables change, as for instance the gluon mass (X.4). Different values of $|a|$ correspond to physically inequivalent vacua. The space they expand is called the moduli space. It would be nice to have a gauge invariant parameterization of such an additional parameter of the gauge invariant vacuum. It can only come from the vacuum expectation value of some gauge invariant operator, since it is an independent new classical parameter which does not appear in the bare Lagrangian. The simplest choice is to take the following gauge invariant chiral superfield:

$$
M=Q \tilde{Q} .
$$

Classically, its vacuum expectation value is 


$$
\langle M\rangle=|a|^{2},
$$

a gauge invariant statement and a good parameterization of the flat direction.

There is one consequence of the flat directions in supersymmetric gauge theories that, when combined with the property of holomorphy, will be important to obtain exact results in supersymmetric theories. SQCD depends of the complex coupling $\tau(\mu)=\theta(\mu) / 2 \pi+4 \pi i / g^{2}(\mu)$ at scale $\mu$. The angle $\theta(\mu)$ measures the strength of CP violation at scale $\mu$. By asymptotic freedom, the theory is weakly coupled at scales higher than the dynamically generated scale $|\Lambda|$, which is defined by

$$
\Lambda \equiv \mu_{0} e^{\frac{2 \pi i \tau\left(\mu_{0}\right)}{b_{0}}},
$$

where $\mu_{0}$ is the ultraviolet cut-off where the bare parameter $\tau_{0}=\tau\left(\mu_{0}\right)$ is defined, and $b_{0}$ is the one-loop coefficient of the beta function,

$$
\mu \frac{\partial g}{\partial \mu}(\mu)=g\left(-b_{0}\left(g^{2} / 16 \pi^{2}\right)+\mathcal{O}\left(g^{4}\right)\right) .
$$

The complex parameter $\Lambda$ is renormalization group invariant in the scheme of the Wilsonian effective actions, where holomorphy is not lost (see below). Observe also that the bare instanton angle $\theta_{0}$ plays the role of the complex phase of $\Lambda^{b_{0}}$.

At scales $\mu \leq \mathcal{M}_{g}$ all the gluons decouple and the relevant degrees of freedom are those of the 'meson' $M$. Its self-interactions are completely determined by the 'microscopic' degrees of freedom of the super-gluons and super-quarks. We must perform a matching condition for the physics at some scale of order $\mathcal{M}_{g}$; the renormalization group will secure the physical equivalence at the other energies. If $\mathcal{M}_{g} \gg \Lambda$, this matching takes place at weak coupling, where perturbation theory in the gauge coupling $g$ is reliable, and we can trust the semiclassical arguments, like those leading to formulae (X.4) and (X.6).

So far we have shown the existence of a flat direction at the classical level. When quantum corrections are included, the flat direction may disappear and a definite value of $\langle M\rangle$ is selected. For the Wilsonian effective description in terms of the relevant degrees of freedom $M$, this is only possible if a superpotential $\mathcal{W}(M)$ is dynamically generated for $M$. By the perturbative nonrenormalization theorem, this superpotential can only be generated by nonperturbative effects, since classically there was no superpotential for the massless gauge singlet $M$ because of the masslessness of the quark multiplet.

If we turn on a bare mass for the quarks, $m$, the flat direction is lifted at classical level and a determined value of mass dependent function $\langle M\rangle$ is selected. But the advantage of the flat direction to carry $\langle M\rangle \rightarrow \infty$ to be at weak coupling is not completely lost. This limit can now be performed by sending the free parameter $m$ to the appropriate limit, as far as we are able to know the mass dependence of the vacuum expectation value of the meson superfield $M$. Here holomorphy is very relevant.

\section{B. Wilsonian effective actions and holomorphy.}

The concept of Wilsonian effective action is simple. Any physical process has a typical scale. The idea of the Wilsonian effective action is to give the Lagrangian of some physical processes at its corresponding characteristic scale $\mu$ :

$$
\mathcal{L}^{(\mu)}(x)=\sum_{i} g^{i}(\mu) \mathcal{O}_{i}(x, \mu) .
$$

$\mathcal{O}_{i}(x, \mu)$ are some relevant local composite operators of the effective fields $\varphi_{a}(p, \mu)$. These are the effective degrees of freedom at scale $\mu$, with momentum modes $p$ running from zero to $\mu$. There could be some symmetries in the operators $\mathcal{O}_{i}$ that our physical system could realize in some way, broken or unbroken. The constants $g^{i}(\mu)$ measure the strength of the interaction $\mathcal{O}_{i}$ of $\varphi_{a}$ at scale $\mu$.

Behind some macroscopic physical processes, there is usually a microscopic theory, with a bare Lagrangian $\mathcal{L}^{\left(\mu_{0}\right)}(x)$ defined at scale $\mu_{0}$. The microscopic theory has also its characteristic scale $\mu_{0}$, much higher than the low energy scale $\mu$. Also its corresponding microscopic degrees of freedom, $\phi_{j}\left(p, \mu_{0}\right)$, may be completely different than the macroscopic ones $\varphi_{a}(p, \mu)$. The bare Lagrangian encodes the dynamics at scales below the ultraviolet cutoff $\mu_{0}$. The effective Lagrangian (X.9) is completely determined by the microscopical Lagrangian $\mathcal{L}^{\left(\mu_{0}\right)}(x)$. It is obtained by integrating out the momentum modes $p$ between $\mu$ and $\mu_{0}$. It gives the values of the effective couplings in terms of the bare couplings $g_{0}^{i}\left(\mu_{0}\right)$,

$$
g^{i}(\mu)=g^{i}\left(\mu ; \mu_{0}, g_{0}^{i}\left(\mu_{0}\right)\right) .
$$

In the macroscopic theory there is no reference to the scale $\mu_{0}$. Physics is independent of the ultraviolet cut-off $\mu_{0}$ :

$$
\frac{\partial g^{i}}{\partial \mu_{0}}=0
$$

The $\mu_{0}$-dependence on the bare couplings $g_{0}^{i}\left(\mu_{0}\right)$ cancel the explicit $\mu_{0}$-dependence in $(\mathrm{X} .10)$. This is the action of the renormalization group. It allows to perform the continuum limit $\mu_{0} \rightarrow \infty$ without changing the low energy physics.

In supersymmetric theories, there are some operators $\mathcal{O}_{i}(z)$, depending only on $\underline{z}=(x, \theta)$, the chiral superspace coordinate, not on $\bar{\theta}$. Clearly, their field content can only be made of chiral superfields. Those of most relevant physical importance are the superpotential $\mathcal{W}\left(\Phi_{i}, \tau_{0}, m_{f}\right)$, and the gauge kinetic operator $\tau\left(\mu / \mu_{0}, \tau_{0}\right) W^{\alpha} W_{\alpha}$. We say that the superpotential $\mathcal{W}$ and the effective gauge coupling $\tau$ are holomorphic functions, with the chiral superfields $\Phi_{i}$, the dimensionless quotient $\mu / \mu_{0}$ and the bare parameters $\tau_{0}$ and $m_{f}$ playing the role of the complex variables. The Kähler potential $K\left(\Phi^{\dagger}, \Phi\right)$ is a real function of the variables $\Phi_{i}$, but 
as far as supersymmetry is not broken and the theory is not on some Coulomb phase, the vacuum structure is determined by the superpotential in the limit $\mu \rightarrow 0$.

We know that complex analysis is substantially more powerful than real analysis. For instance, there are a lot of real functions $f(x)$ that at $x \rightarrow 0$ and $x \rightarrow \infty$ go like $f(x) \rightarrow x$. But there is only one holomorphic function $f(z)\left(\partial_{\bar{z}} f(z)=0\right)$ with those properties: $f(z)=z$. The holomorphic constraint is so strong that sometimes the symmetries of the theory, together with some consistency conditions, are enough to determine the unique possible form of the functions $\mathcal{W}$ and $\tau$ 24.

An illustrative example is the saturation at one-loop of the holomorphic gauge coupling $\tau\left(\mu / \mu_{0}, \tau_{0}\right)$ at any order of perturbation theory. Since $\tau_{0}=\theta_{0} / 2 \pi+i 4 \pi / g_{0}^{2}$, physical periodicity in $\theta_{0}$ implies

$$
\tau\left(\frac{\mu}{\mu_{0}}, \tau_{0}\right)=\tau_{0}+\sum_{n=0}^{\infty} c_{n}\left(\frac{\mu}{\mu_{0}}\right) e^{2 \pi n i \tau_{0}},
$$

where the sum is restricted to $n \geq 0$ to ensure a well defined weak coupling limit $g_{0} \rightarrow 0$. The unique term compatible with perturbation theory is the $n=0$ term. Terms with $n>0$ corresponds to instanton contributions. The function $c_{0}(t)$ must satisfy $c_{0}\left(t_{1} t_{2}\right)=c_{0}\left(t_{1}\right)+c_{0}\left(t_{2}\right)$ and hence it must be a logarithm. Hence

$$
\tau_{\text {pert }}\left(\frac{\mu}{\mu_{0}}, \tau_{0}\right)=\tau_{0}+\frac{i b_{0}}{2 \pi} \ln \frac{\mu}{\mu_{0}},
$$

with $b_{0}$ the one-loop coefficient of the beta function. We can use the definition $\mathrm{X} .7$ ) of the dynamically generated scale $\Lambda$ to absorb the bare coupling constant inside the logarithm

$$
\tau_{\text {pert }}\left(\frac{\mu}{\Lambda}\right)=\frac{i b_{0}}{2 \pi} \ln \frac{\mu}{\Lambda}
$$

showing explicitly the independence of the effective gauge coupling in the ultraviolet cut-off $\mu_{0}$.

We would like to comment that the one-loop saturation of the perturbative beta function and the renormalization group invariance of the scale $\Lambda$ can be lost by the effect of the Konishi anomaly [25,26]. In general, after the integration of the modes $\mu<p<\mu_{0}$ the kinetic terms of the matter fields $\Phi_{i}$ are not canonically normalized,

$$
\mathcal{L}^{(\mu)}=\sum_{i} Z_{i}\left(\frac{\mu}{\mu_{0}}, g_{0}\right) \int d^{4} \theta \Phi_{i}^{\dagger} e^{-2 V} \Phi_{i}+\cdots
$$

These terms have an integral on the whole superspace $(\theta, \bar{\theta})$ and hence are not protected by any nonrenormalization theorem. For $N=1$ gauge theories, holomorphy is absent there, and the functions $Z_{i}\left(\frac{\mu}{\mu_{0}}, g_{0}\right)$ are just real functions with perturbative multi-loops contributions. A canonical normalization of the matter fields in the effective action, defining the canonical fields $\Phi_{i}^{\prime}=Z_{i}^{1 / 2} \Phi_{i}$ do not leaves invariant the path integral measure $\Pi_{i} \mathcal{D} \Phi_{i}$. The anomaly is proportional to $\left(\sum_{i} \ln Z_{i}\right) W^{\alpha} W_{\alpha}$, giving a non-holomorphic contribution to the effective coupling $\tau$. For $N=2$ theories, $Z_{i}=1$ and holomorphy is not lost for $\tau$ 26, 27.

\section{XI. $N=1$ SQCD.}

\section{A. Classical Lagrangian and symmetries.}

We now analyze $N=1$ SQCD with gauge group $S U\left(N_{c}\right)$ and $N_{f}$ flavors **. The field content is the following: There is a spinor chiral superfield $W_{\alpha}$ in the adjoint of $S U\left(N_{c}\right)$, which contains the gluons $A_{\mu}$ and the gluinos $\lambda$. The matter content is given by $2 N_{f}$ scalar chiral superfields $Q_{f}$ and $\tilde{Q}_{f}, f, \widetilde{f}=1, \ldots, N_{f}$, in the $\mathbf{N}_{\mathbf{c}}$ and $\overline{\mathbf{N}}_{\mathbf{c}}$ representations of $S U\left(N_{c}\right)$ respectively. The renormalizable bare Lagrangian is the following:

$$
\begin{aligned}
\mathcal{L}_{\mathcal{S Q C D}} & =\frac{1}{8 \pi} \operatorname{Im}\left(\tau_{0} \int d^{2} \theta W^{\alpha} W_{\alpha}\right) \\
& +\int d^{4} \theta\left(Q_{f}^{\dagger} e^{-2 V} Q_{f}+\tilde{Q}_{f} e^{2 V} \tilde{Q}_{f}^{\dagger}\right) \\
& +\left(\int d^{2} \theta m_{f} \tilde{Q}_{f} Q_{f}+\text { h.c. }\right)
\end{aligned}
$$

with $\tau_{0}=\theta_{0} / 2 \pi+i 4 \pi / g_{0}^{2}$ and $m_{f}$ the bare couplings. In the massless limit the global symmetry of the classical Lagrangian is $S U\left(N_{f}\right)_{L} \times S U\left(N_{f}\right)_{R} \times U(1)_{B} \times U(1)_{A} \times$ $U(1)_{R}$. For $N_{c}=2$ the representations $\mathbf{2}$ and $\overline{\mathbf{2}}$ are equivalent, and the global symmetry group is enlarged. In general we consider $N_{c}>2$. The $U(1)_{A}$ and $U(1)_{R}$ symmetries are anomalous and are broken by instanton effects. But we can perform a linear combination of $U(1)_{A}$ and $U(1)_{R}$, call it $U(1)_{A F}$, that is anomaly free. We have the following table of representations for the global symmetries of SQCD:

\begin{tabular}{c|c|c|c|c} 
& $S U\left(N_{f}\right)_{L}$ & $S U\left(N_{f}\right)_{R}$ & $U(1)_{B}$ & $U(1)_{A F}$ \\
\hline$W_{\alpha}$ & $\mathbf{1}$ & $\mathbf{1}$ & 0 & 1 \\
$Q_{f}$ & $\mathbf{N}_{\mathbf{c}}$ & $\mathbf{1}$ & 1 & $\frac{\left(N_{f}-N_{c}\right)}{N_{f}}$ \\
$\tilde{Q}_{f}$ & $\mathbf{1}$ & $\overline{\mathbf{N}}_{\mathbf{c}}$ & -1 & $\frac{\left(N_{f}-N_{c}\right)}{N_{f}}$
\end{tabular}

The anomaly free $R$-charges, $R_{A F}$, are derived by the following. The superfield $W_{\alpha}$ is neutral under $U(1)_{A}$ and its R-transformation is fixed to be

$$
W_{\alpha}(x, \theta) \rightarrow e^{i \beta} W_{\alpha}\left(x, e^{-i \beta} \theta\right) .
$$

\footnotetext{
${ }^{* *}$ Some reviews on exacts results in $N=1$ supersymmetric gauge theories are 28 .
} 
Consider now that the fermionic quarks $\psi$ have charge $R_{\psi}$ under an $U(1)_{A F}$ transformation. In the one-instanton sector, $\lambda$ has $2 N_{c}$ zero modes, and one for each $Q_{f}$ and $\tilde{Q}_{f}$. In total we have $2 N_{c}+2 N_{f} R_{\psi}=0$ to avoid the anomalies. We derive that $R_{\psi}=-N_{c} / N_{f}$. Since this is the charge of the fermions, the superfields $\left(Q_{f}, \tilde{Q}_{f}\right)$ have $R_{A F}$ charge $1-N_{c} / N_{f}=\left(N_{f}-N_{c}\right) / N_{f}$.

\section{B. The classical moduli space.}

The classical equations of motion of the auxiliary fields are

$$
\begin{aligned}
& \bar{F}_{q_{f}}=-m_{f} \tilde{q}_{f}=0, \\
& \bar{F}_{\tilde{q}_{f}}=-m_{f} q_{f}=0, \\
& D^{a}=\sum_{f}\left(q_{f}^{\dagger} T^{a} q_{f}-\tilde{q}_{f} T^{a} \tilde{q}_{f}^{\dagger}\right)=0 .
\end{aligned}
$$

If there is a massive flavor $m_{f} \neq 0$, then we must have $q_{f}=\tilde{q}_{f}=0$. As we want to go to the infrared limit to analyze the vacuum structure, the interesting case is the situation of $N_{f}$ massless flavors. If some quark has a nonzero mass $m$, its physical effects can be decoupled at very low energy, by taking into account the appropriate physical matching conditions at the decoupling scale $m$ (see below). If all quarks are massive, in the infrared limit we only have a pure $S U\left(N_{c}\right)$ supersymmetric gauge theory. The Witten index of pure $S U\left(N_{c}\right)$ super Yang-Mills is $\operatorname{tr}(-1)^{F}=N_{c}$ 29. We know that supersymmetry is not broken dynamically in this theory, and that there are $N_{c}$ equivalent vacua. The $2 N_{c}$ gaugino zero modes break the $U(1)_{R}$ symmetry to $Z_{2 N_{c}}$ by the instantons. Those $N_{c}$ vacua corresponds to the spontaneously broken discrete symmetry $Z_{2 N_{c}}$ to $Z_{2}$ by the gaugino condensate $\langle\lambda \lambda\rangle \neq 0$.

If there are some massless super-quarks, they can have non-trivial physical effects on the vacuum structure. Consider that we have $N_{f}$ massless flavors. We can look at the $q_{f}$ and $\tilde{q}_{f}$ scalar quarks as $N_{c} \times N_{f}$ matrices. Using $S U\left(N_{c}\right) \times S U\left(N_{f}\right)$ transformations, the $q_{f}$ matrix can be rotated into a simple form. There are two cases to be distinguished:

a) $N_{f}<N_{c}$ :

In this case we have that the general solution of the classical vacuum equations (XI.3) is:

$$
q_{f}=\tilde{q}_{f}^{\dagger}=\left(\begin{array}{cccc}
v_{1} & 0 & \cdots & 0 \\
0 & v_{2} & & \\
& & \ddots & \\
0 & \ldots & & v_{N_{f}} \\
\vdots & & & \vdots \\
0 & \ldots & & 0
\end{array}\right)
$$

with $v_{f}$ arbitrary. These scalar quark's vacuum expectation values break spontaneously the gauge group to
$S U\left(N_{c}-N_{f}\right)$. By the super-Higgs mechanism, $N_{c}^{2}-\left(N_{c}-\right.$ $\left.N_{f}\right)^{2}=2 N_{c} N_{f}-N_{f}^{2}$ chiral superfields are eaten by the vector superfields. This leaves $2 N_{f} N_{c}-\left(2 N_{f} N_{c}-N_{f}^{2}\right)=$ $N_{f}^{2}$ chiral superfields. They can be described by the meson operators

$$
M_{f g} \equiv \tilde{Q}_{f} Q_{g}
$$

which provide a gauge invariant description of the classical moduli space.

b) $N_{f} \geq N_{c}$ :

In this case the general solution of (XI.3) is:

$$
\begin{gathered}
q_{f}=\left(\begin{array}{cccccc}
v_{1} & 0 & \cdots & 0 & \cdots & 0 \\
0 & v_{2} & & \vdots & & \vdots \\
& & \ddots & & & \vdots \\
& & & v_{N_{c}} & \cdots & 0
\end{array}\right), \\
\tilde{q}_{f}^{\dagger}=\left(\begin{array}{cccccc}
\tilde{v}_{1} & 0 & \cdots & 0 & \cdots & 0 \\
0 & \tilde{v}_{2} & & \vdots & & \vdots \\
& & \ddots & & & \vdots \\
& & & \tilde{v}_{N_{c}} & \cdots & 0
\end{array}\right),
\end{gathered}
$$

with the parameters $v_{i}, \tilde{v}_{i}\left(i=1, \ldots, N_{c}\right)$ subject to the constraint

$$
\left|v_{i}\right|^{2}-\left|\tilde{v}_{i}\right|^{2}=\text { constant independent of } i \text {. }
$$

Now the gauge group is completely higgsed. The gauge invariant parameterization of the classical moduli space must be done by $2 N_{f} N_{c}-\left(N_{c}^{2}-1\right)$ chiral superfields. For instance, if $N_{f}=N_{c}$, we need $N_{c}^{2}+1$ superfields. The meson operators $M_{f g}$ provide $N_{c}^{2}$. The remaining degree of freedom comes from the baryon-like operators

$$
\begin{aligned}
& B=\epsilon^{f_{1} \cdots f_{N_{f}}} Q_{f_{1}} \cdots Q_{f_{N_{f}}}, \\
& \tilde{B}=\epsilon^{f_{1} \cdots f_{N_{f}}} \tilde{Q}_{f_{1}} \cdots \tilde{Q}_{f_{N_{f}}},
\end{aligned}
$$

with the color indices also contracted by the $\epsilon$-tensor. These are two superfields, but there is a holomorphic constraint

$$
\operatorname{det} M-\tilde{B} B=0 \text {. }
$$

For $N_{f}=N_{c}+1$, we need $2 N_{c}\left(N_{c}+1\right)-\left(N_{c}^{2}-1\right)=$ $N_{c}^{2}+2 N_{c}+1$ independent chiral superfields. We can construct the baryon operators:

$$
\begin{aligned}
& B^{f}=\epsilon^{f f_{1} \cdots f_{N_{c}}} Q_{f_{1}} \cdots Q_{f_{N_{c}}}, \\
& \tilde{B}^{f}=\epsilon^{f f_{1} \cdots f_{N_{c}}} \tilde{Q}_{f_{1}} \cdots \tilde{Q}_{f_{N_{c}}} .
\end{aligned}
$$

$M_{f g}, B^{f}$ and $\tilde{B}^{f}$ have $\left(N_{c}+1\right)^{2}+2\left(N_{c}+1\right)$ components. The matrix $M_{f g}$ has rank $N_{c}$, which can be expressed by the $2\left(N_{c}+1\right)$ constraints:

$$
M_{f g} B^{g}=M_{f g} \tilde{B}^{g}=0 .
$$

And in total we get the needed $N_{c}^{2}+2 N_{c}+1$ independent chiral superfields.

As $N_{f}$ increases, we get more and more constraints. Each case with $N_{f} \geq N_{c}$ is interesting by itself and we will have to look at them in different ways. 


\section{THE VACUUM STRUCTURE OF SQCD WITH $N_{F}<N_{C}$.}

\section{A. The Afleck-Dine-Seiberg's superpotential.}

First we consider the case of massless flavors. At the classical level there are flat directions parameterized by the free vacuum expectation values of the meson fields $M_{f g}$. They belong to the representation $\left(\mathbf{N}_{\mathbf{f}}, \overline{\mathbf{N}}_{\mathbf{f}}, 0,2\left(N_{f}-N_{c}\right) / N_{f}\right)$ of the global symmetry group $S U\left(N_{f}\right)_{L} \times S U\left(N_{f}\right)_{R} \times U(1)_{B} \times U(1)_{A F}$. If nonperturbative effects generate a Wilsonian effective superpotential $\mathcal{W}$, it must depend in a holomorphic way of the light chiral superfields $M_{f g}$ and the bare coupling constant $\tau_{0}$. The renormalization group invariance of the Wilsonian effective action demands that the dependence on the bare coupling constant $\tau_{0}$ of $\mathcal{W}$ enters thought the dynamically generated scale $\Lambda_{N_{f}, N_{c}}$. The invariance of $\mathcal{W}$ under $S U\left(N_{f}\right)_{L} \times S U\left(N_{f}\right)_{R}$ rotations reduces the dependence in the mesons fields to the combination $\operatorname{det} M$. There is only one holomorphic function $\mathcal{W}=\mathcal{W}\left(\operatorname{det} M, \Lambda_{N_{f}, N_{c}}\right)$, with $R_{A F}$ charge two that can be built from the variables $\operatorname{det} M$ and $\Lambda_{N_{f}, N_{c}}$, which have $R_{A F}$ charge $2\left(N_{f}-N_{c}\right)$ and zero, respectively. It is the Afleck-Dine-Seiberg's superpotential 30,31.

$$
\mathcal{W}=c_{N_{f}, N_{g}}\left(\frac{\Lambda_{N_{f}, N_{c}}}{\operatorname{det} M}\right)^{\frac{1}{\left(N_{c}-N_{f}\right)}}
$$

where $c_{N_{f}, N_{c}}$ are some undetermined dimensionless constants. If $c_{N_{f}, N_{c}} \neq 0$, (XII.1) corresponds to an exact nonperturbative dynamically generated Wilsonian superpotential. It has catastrophic consequences, the theory has no vacuum. If we try to minimize the energy derived from the superpotential (XII.1) we find that $|\langle\operatorname{det} M\rangle| \rightarrow \infty$.

\section{B. Massive flavors.}

When we add mass terms for all the flavors we expect to find some physical vacua. In fact, by Witten index, we should find $N_{c}$ of them. To verify this, let us try to compute $\left\langle M_{f g}\right\rangle$ taking advantage of its holomorphy and symmetries.

A bare mass matrix $m_{f g} \neq 0$ breaks explicitly the $S U\left(N_{f}\right) \times S U\left(N_{f}\right)_{R} \times U(1)_{A F}$ global symmetry of the bare Lagrangian (XI.1). In terms of the meson operator the mass term is

$$
\mathcal{W}_{\text {tree }}=\operatorname{tr}(m M)
$$

We see that, under an $L$ and $R$ rotation of $S U\left(N_{f}\right)_{L}$ and $S U\left(N_{f}\right)_{R}$ respectively, we can recover the $S U\left(N_{f}\right)_{L} \times$ $S U\left(N_{f}\right)_{R}$ invariance if we require $m$ to transform as $m \rightarrow L^{-1} m R$. In the same way, as the superpotential has R-charge two, the $U(1)_{A F}$ invariance is recovered if we assign the charge $2-2\left(N_{f}-N_{c}\right) / N_{f}=2 N_{c} / N_{f}$ to the mass matrix $m$. The vacuum expectation value of the matrix chiral superfield $M$ is a holomorphic function of $\Lambda_{N_{f}, N_{c}}$ and $m$. To implement the same action under $S U\left(N_{f}\right)_{L} \times S U\left(N_{f}\right)_{R}$ rotations, we must have

$$
\langle M\rangle=f\left(\operatorname{det} m, \Lambda_{N_{f}, N_{c}}\right) m^{-1} .
$$

The dependence in $\operatorname{det} m$ of the function $f$ is determined by the $R_{A F}$ charge. Then, the $\Lambda_{N_{f}, N_{c}}$ dependence is worked out by dimensional analysis. The result is

$$
\langle M\rangle=(\text { const })\left(\Lambda_{N_{f}, N_{c}}^{3 N_{c}-N_{f}} \operatorname{det} m\right)^{\frac{1}{N_{c}}} m^{-1} .
$$

The $N_{c}$ roots give $N_{c}$ vacua. Observe that this is an exact result, and valid also for $N_{f} \geq N_{c}$. There is only an dimensionless constant (in general $N_{f}$ and $N_{c}$ dependent) to be determined. It would be nice to be able to carry its computation in the weak coupling limit, since holomorphy would allow to extend (XII.4) also to the strong coupling region.

The result (XII.4 suggest the existence of an effective superpotential out of which XII.4 can be obtained. Holomorphy and symmetries tell us that the possible superpotential would have to be

$$
\begin{aligned}
& \mathcal{W}\left(M, \Lambda_{N_{f}, N_{c}}, m\right)=\left(\frac{\Lambda_{N_{f}, N_{c}}}{\operatorname{det} M}\right)^{\frac{1}{\left(N_{c}-N_{f}\right)}} . \\
& f\left(t=\operatorname{tr}(m M)\left(\frac{\Lambda_{N_{f}, N_{c}}}{\operatorname{det} M}\right)^{\frac{-1}{\left(N_{c}-N_{f}\right)}}\right) .
\end{aligned}
$$

In the limit of weak coupling, $\Lambda_{N_{f}, N_{c}} \rightarrow 0$, we know that $f(t)=c_{N_{f}, N_{c}}+t$. But we can play at the same time with the free values of $m$ to reach any desired value of $t$. This fixes the function $f(t)$ and the superpotential $\mathcal{W}\left(M, \Lambda_{N_{f}, N_{c}}, m\right)$ to be

$$
\begin{aligned}
\mathcal{W}\left(M, \Lambda_{N_{f}, N_{c}}, m\right) & =c_{N_{f}, N_{c}}\left(\frac{\Lambda_{N_{f}, N_{c}}}{\operatorname{det} M}\right)^{\frac{1}{\left(N_{c}-N_{f}\right)}} \\
& +\operatorname{tr}(m M) .
\end{aligned}
$$

As a consistency check, when we solve the equations $\partial \mathcal{W} / \partial M=0$, we obtain the previously determined vacuum expectation values (XII.4).

Finally, we have to check the non-vanishing of $c_{N_{f}, N_{c}}$. We take advantage of the decoupling theorem to obtain further information about the constants $c_{N_{f}, N_{c}}$. Let us add a mass term $m$ only for the $N_{f}$ flavor,

$$
\begin{aligned}
\mathcal{W}\left(M, \Lambda_{N_{f}, N_{c}}, m\right) & =\left(\frac{\Lambda_{N_{f}, N_{c}}}{\operatorname{det} M}\right)^{\frac{1}{\left(N_{c}-N_{f}\right)}} \\
& +m M_{N_{f} N_{f}}
\end{aligned}
$$

Solving for the equations: 


$$
\begin{gathered}
\frac{\partial \mathcal{W}}{\partial M_{f N_{f}}}\left(M, \Lambda_{N_{f}, N_{c}}, m\right)=0, \\
\frac{\partial \mathcal{W}}{\partial M_{N_{f} f}}\left(M, \Lambda_{N_{f}, N_{c}}, m\right)=0,
\end{gathered}
$$

for $f<N_{f}$ gives that $M_{f N_{f}}=M_{N_{f} f}=0$. Hence $\operatorname{det} M=M_{N_{f} N_{f}} \cdot \operatorname{det} \hat{M}$, with $\hat{M}$ the $\left(N_{f}-1\right) \times\left(N_{f}-1\right)$ matrix meson operator of the $N_{f}-1$ massless flavors. At scales below $m$, the $N_{f}$-th flavor decouples and its corresponding $M_{N_{f} N_{f}}$ meson operator is frozen to the value that satisfies:

$\frac{\partial \mathcal{W}}{\partial M_{N_{f} N_{f}}}\left(M, \Lambda_{N_{f}, N_{c}}, m\right)=-\frac{c_{N_{f}, N_{c}}}{\left(N_{f}-N_{c}\right)}$.

$\Lambda_{N_{f}, N_{c}}^{\left(3 N_{f}-N_{c}\right) /\left(N_{f}-N_{c}\right)}(\operatorname{det} M)^{\frac{1}{\left(N_{c}-N_{f}\right)}-1} \operatorname{det} \hat{M}+m=0$.

If we substitute the solution $\left\langle M_{N_{f} N_{f}}\right\rangle$ of the previous equation into the superpotential $\mathcal{W}\left(M, \Lambda_{N_{f}, N_{c}}, m\right)$, we should obtain the superpotential $\mathcal{W}\left(\hat{M}, \Lambda_{N_{f}-1, N_{c}}, 0\right)$ of $N_{f}-1$ massless flavors with the dynamically generated scale $\Lambda_{N_{f}-1, N_{c}}$. The matching conditions at scale $m$ between the theory with $N_{f}$ flavors and the theory with $N_{f}-1$ flavors gives the relation

$$
m \Lambda_{N_{f}, N_{c}}^{3 N_{c}-N_{f}}=\Lambda_{N_{f}-1, N_{c}}^{3 N_{c}-N_{f}+1}
$$

thus,

$$
\begin{aligned}
& \left.\mathcal{W}\left(M, \Lambda_{N_{f}, N_{c}}, m\right)\right|_{\left\langle M_{N_{f} N_{f}}\right\rangle}=\left(N_{c}-N_{f}+1\right) . \\
& \left(\frac{c_{N_{f}, N_{c}}}{N_{c}-N_{f}}\right)^{\frac{N_{c}-N_{f}}{N_{c}-N_{f}+1}}\left(\frac{\Lambda_{N_{f}-1, N_{c}}}{\operatorname{det} \hat{M}}\right)^{\frac{1}{\left(N_{c}-N_{f}+1\right)}},
\end{aligned}
$$

and we obtain the relation

$$
\left(\frac{c_{N_{f}, N_{c}}}{N_{c}-N_{f}}\right)^{N_{c}-N_{f}}=\left(\frac{c_{N_{f}-1, N_{c}}}{N_{c}-N_{f}+1}\right)^{N_{c}-N_{f}+1} .
$$

Similarly, we can try to obtain another relation between the constants $c_{N_{f}, N_{c}}$ for different numbers of colors. To this end we give a large expectation value to $M_{N_{f} N_{f}}$ with respect the expectation values of $\hat{M}$. Then below the scale $\left\langle M_{N_{f} N_{f}}\right\rangle$ we have SQCD with $N_{c}-1$ colors and $N_{f}-1$ flavors. Following the same strategy as before we find that $c_{N_{f}-1, N_{c}-1}=c_{N_{c}, N_{f}}$. It means that $c_{N_{c}, N_{f}}=$ $c_{N_{f}-N_{c}}$, which together with the relation (XII.12) gives

$$
c_{N_{f}, N_{c}}=\left(N_{c}-N_{f}\right) c_{1,2} .
$$

We just have to compute the dimensionless constant $c_{1,2}$ of the gauge group $S U(2)$ with one flavor. In this case, or for the general case of $N_{f}=N_{c}-1$, the gauge group is completely higgsed and there are not infrared divergences in the instanton computation. In the weak coupling limit the unique surviving nonperturbative contributions come from the one-instanton sector. A direct instanton calculation reveals that the constant $c_{2,1} \neq 0$ 31]

For $N_{f}<N_{c}-1$ there is an unbroken gauge group $S U\left(N_{c}-N_{f}\right)$. At scales below the smallest eigenvalue of the matrix $\left\langle M_{f g}\right\rangle$ we have a pure super Yang-Mills theory with $N_{c}-N_{f}$ colors. This theory is believed to confine with a mass gap given by the gaugino condensate $\langle\lambda \lambda\rangle \neq 0$. Consider the simplest case of $\left\langle M_{f g}\right\rangle=\mu^{2} \mathbf{1}_{N_{f}}$. Matching the gauge couplings at scale $\mu$ gives $\Lambda_{N_{f}, N_{c}}^{3 N_{c}-N_{f}}=$ $(\operatorname{det} M) \Lambda_{0, N_{c}-N_{f}}^{3\left(N_{c}-N_{f}\right)}$, which implies for the effective superpotential

$$
\mathcal{W}=\left(N_{c}-N_{f}\right) \Lambda_{0, N_{c}-N_{f}}^{3}
$$

On the other hand, the gaugino bilinear $\lambda \lambda$ is the lowest component of the chiral superfield $S=W^{\alpha} W_{\alpha}$, which represents the super-glueball operator. The bare gauge coupling $\tau_{0}$ acts as the source of the operator $S$. If we differentiate (XII.14) with respect to $\ln \Lambda^{3\left(N_{c}-N_{f}\right)}$ we obtain the gaugino condensate

$$
\langle\lambda \lambda\rangle=\Lambda_{0, N_{c}-N_{f}}^{3}
$$

In fact, following the 'integrating in' procedure [33,34], we would obtain the Veneziano-Yankielowicz effective Lagrangian [35].

It is not possible to extend the Afleck-Dine-Seiberg's superpotential to the case of $N_{f} \geq N_{c}$. For these values the quantum corrections do not lift the flat directions, and we still have a moduli space which may be different from the classical one. This is the case of $N_{f}=N_{c}$.

\section{THE VACUUM STRUCTURE OF SQCD WITH $N_{F}=N_{C}$.}

\section{A. A quantum modified moduli space.}

For $N_{f}=N_{c}$, the classical moduli space is spanned by the gauge singlet operators $M_{f g}, B$ and $\tilde{B}$ subject to the constraint $\operatorname{det} M-\tilde{B} B=0$. At quantum level, instanton effects could change the classical constraint to

$$
\operatorname{det} M-\tilde{B} B=\Lambda^{2 N_{c}},
$$

since $\Lambda^{2 N_{c}} \sim e^{-8 \pi / g^{2}+i \theta}$ corresponds to the oneinstanton factor, it has the right dimensions, and the operators $\left(Q_{f}, \tilde{Q}_{f}\right)$ have $R_{A F}$ charge zero.

To check if the quantum correction (XIII.1) really takes place, add a mass term for the quarks. The unique possible holomorphic term with $R_{A F}$ charge two that can be generated with the variables $\left(M_{f g}, B, \tilde{B}, \Lambda, m\right)$ is

\footnotetext{
${ }^{\dagger \dagger}$ In the $\overline{\mathrm{DR}}$ scheme $c_{2,1}=1[32$. If we do not say the contrary, we will work on such a scheme.
} 


$$
\mathcal{W}=\operatorname{tr} m M
$$

Imagine now that the $N_{c}$-flavor is much heavier, with bare mass $m$, than the $N_{c}-1$ other ones, with bare mass matrix $\hat{m}$. The degree of freedom $M_{N_{c} N_{c}}$ is given by the constraint. Locate at $B=\tilde{B}=M_{f N_{c}}=0$. By equation (XII.4) we know that the $\left(N_{c}-1\right) \times\left(N_{c}-1\right)$ matrix $\hat{M}$ is determined to be

$$
\hat{M}=\left(\Lambda_{N_{c}-1, N_{c}}^{2 N_{c}+1} \operatorname{det} \hat{m}\right)^{\frac{1}{N_{c}}} \hat{m}^{-1}
$$

which has a non-zero determinant. It indicates that the constraint XIII.1 is really generated at quantum level 36. As a final check, consider the simplest situation of $N_{c}-1$ massless flavors. When we use the constraint (XIII.1) to express $M_{N_{c} N_{c}}$ as function of $\operatorname{det} \hat{M}$ we obtain

$$
\mathcal{W}=\frac{m \Lambda^{2 N_{c}}}{\operatorname{det} \hat{M}}
$$

the Afleck-Dine-Seiberg's superpotential for $N_{f}=N_{c}-1$ massless flavors.

Far from the origin of the moduli field space we are at weak coupling and the quantum moduli space given by the constraint (XIII.1) looks like the classical moduli space (XI.10). But far from the origin of order $\Lambda$, the one-instanton sector is sufficiently strong to change significatively the vacuum structure. Observe that the classically allowed point $M=B=\tilde{B}=0$ is not a point of the quantum moduli space and the gluons never become massless.

\section{B. Patterns of spontaneous symmetry breaking and 't Hooft's anomaly matching conditions.}

Our global symmetries are $S U\left(N_{f}\right)_{L} \times S U\left(N_{f}\right)_{R} \times$ $U(1)_{B} \times U(1)_{A F}$. Since for $N_{f}=N_{c}$ the super-quarks are neutral with respect to the non-anomalous symmetry $U(1)_{A F}$, it is never spontaneously broken. The other symmetries present different patterns of symmetry breaking depending on which point of the moduli space the vacuum is located 周.

For instance, the point

$$
M=\Lambda^{2} \mathbf{1}_{N_{f}}, \quad B=\tilde{B}=0,
$$

suggests the spontaneous symmetry breaking

$$
\begin{aligned}
& S U\left(N_{f}\right)_{L} \times S U\left(N_{f}\right)_{R} \times U(1)_{B} \times U(1)_{A F} \\
& \longrightarrow S U\left(N_{f}\right)_{V} \times U(1)_{B} \times U(1)_{A F}
\end{aligned}
$$

\footnotetext{
${ }^{\ddagger \ddagger}$ Different patterns of symmetry breaking have also been observed in softly broken $N=2$ SQCD 15.
}

with $S U\left(N_{f}\right)_{V}$ the diagonal part of $S U\left(N_{f}\right) \times S U\left(N_{f}\right)_{R}$. To check it, the unbroken symmetries must satisfy the 't Hooft's anomaly matching conditions [37].

With respect to the unbroken symmetries the quantum numbers of the elementary and composite massless fermions, at high and low energy respectively, are

\begin{tabular}{c|c|c|c|} 
& $S U\left(N_{f}\right)_{V}$ & $U(1)_{B}$ & $U(1)_{A F}$ \\
\hline$\lambda$ & $\mathbf{1}$ & 0 & 1 \\
$\psi_{q}$ & $\mathbf{N}_{\mathbf{f}}$ & 1 & -1 \\
$\psi_{\tilde{q}}$ & $\overline{\mathbf{N}}_{\mathbf{f}}$ & -1 & -1 \\
\hline & & & \\
$\psi_{M}$ & $\mathbf{N}_{\mathbf{f}}^{\mathbf{2}}-\mathbf{1}$ & 0 & -1 \\
$\psi_{B}$ & $\mathbf{1}$ & $N_{f}$ & -1 \\
$\psi_{\tilde{B}}$ & $\mathbf{1}$ & $-N_{f}$ & -1
\end{tabular}

Observe there are only $N_{f}^{2}-1$ independent meson fields, arranged in the adjoint of $S U\left(N_{f}\right)_{V}$, since the constraint (XIII.1) eliminates one of them. There are $N_{f}^{2}-1$ gluinos and $N_{f}$ extra components for each quark $\psi_{q}$ and antiquark $\psi_{\tilde{q}}$ because of the gauge group $S U\left(N_{c}\right)$. The anomaly coefficients are:

\begin{tabular}{c|c|c|} 
triangles & high energy & low energy \\
\hline$S U\left(N_{f}\right)^{2} \times U(1)_{A F}$ & $-2 N_{f} T\left(\mathbf{N}_{\mathbf{f}}\right)$ & $-T\left(\mathbf{N}_{\mathbf{f}}^{\mathbf{2}}-\mathbf{1}\right)$ \\
$U(1)_{A F}^{3}$ & $-2 N_{f}^{2}+\left(N_{f}^{2}-1\right)$ & $-\left(N_{f}^{2}-1\right)-2$ \\
$U(1)_{B}^{2} \times U(1)_{A F}$ & $-N_{f}^{2}-N_{f}^{2}$ & $-2 N_{f}^{2}$ \\
$\operatorname{tr} U(1)_{A F}$ & $-2 N_{f}^{2}+N_{f}^{2}-1$ & $-\left(N_{f}^{2}-1\right)-2$
\end{tabular}

The constants $T(R)$ are defined by $\operatorname{tr}\left(T^{a} T^{a}\right)=$ $T(R) \delta^{a b}$, with $T^{a}$ in the representation $R$ of the group $S U(N)$. For the fundamental representation, $T(\mathbf{N})=$ $1 / 2$. For the adjoint representation, $T\left(\mathbf{N}^{\mathbf{2}}-\mathbf{1}\right)=N$. The coefficient of $\operatorname{tr} U(1)_{A F}$ corresponds to the gravitational anomaly. One can check that all the anomalies match perfectly, supporting the spontaneous symmetry breaking pattern of (XIII.6).

The quantum moduli space of $N_{f}=N_{c}$ allows another particular point with a quite different breaking pattern. It is:

$$
M=0, \quad B=-\tilde{B}=\Lambda^{N_{c}} .
$$

At this point, only the vectorial baryon symmetry is broken, all the chiral symmetries $S U\left(N_{f}\right)_{L} \times S U\left(N_{f}\right)_{R} \times$ $U(1)_{A F}$ remain unbroken. We check this pattern with the help of the 't Hooft's anomaly matching conditions again. In this case we have the quantum numbers: 


\begin{tabular}{c|c|c|c|} 
& $S U\left(N_{f}\right)_{L}$ & $S U\left(N_{f}\right)_{R}$ & $U(1)_{A F}$ \\
\hline$\lambda$ & $\mathbf{1}$ & $\mathbf{1}$ & 1 \\
$\psi_{q}$ & $\mathbf{N}_{\mathbf{f}}$ & $\mathbf{1}$ & -1 \\
$\psi_{\tilde{q}}$ & $\mathbf{1}$ & $\overline{\mathbf{N}}_{\mathbf{f}}$ & -1 \\
\hline$\psi_{M}$ & $\mathbf{N}_{\mathbf{f}}$ & $\overline{\mathbf{N}}_{\mathbf{f}}$ & -1 \\
$\psi_{B}$ & $\mathbf{1}$ & $\mathbf{1}$ & -1 \\
$\psi_{\tilde{B}}$ & $\mathbf{1}$ & $\mathbf{1}$ & -1
\end{tabular}

and the anomaly coefficients are:

\begin{tabular}{c|c|c|} 
triangles & high energy & low energy \\
\hline & & \\
$S U\left(N_{f}\right)_{L}^{3}$ & $N_{f} C_{3}$ & $N_{f} C_{3}$ \\
$S U\left(N_{f}\right)_{R}^{3}$ & $N_{f} C_{3}$ & $N_{f} C_{3}$ \\
$S U\left(N_{f}\right)^{2} \times U(1)_{A F}$ & $-N_{f} T\left(\mathbf{N}_{\mathbf{f}}\right)$ & $-N_{f} T\left(\mathbf{N}_{\mathbf{f}}\right)$ \\
$U(1)_{A F}^{3}$ & $-2 N_{f}^{2}+N_{f}^{2}-1$ & $-N_{f}^{2}-1$
\end{tabular}

where $C_{3}$ is defined by $\operatorname{tr}\left(T^{a}\left\{T^{b}, T^{c}\right\}\right)=C_{3} d^{a b c}$, with $T^{a}$ in the fundamental representation of $S U\left(N_{f}\right)$. Because of the constraint XIII.1 there is only one independent baryonic degree of freedom. The anomaly coefficients match perfectly.

\section{THE VACUUM STRUCTURE OF SQCD WITH $N_{F}=N_{C}+1$.}

\section{A. The quantum moduli space.}

First we consider if the classical constraints:

$$
\begin{aligned}
M_{f g} B^{g}=M_{f g} \tilde{B}^{f} & =0, \\
\operatorname{det} M\left(M^{-1}\right)^{f g}-B^{f} \widetilde{B}^{g} & =0,
\end{aligned}
$$

are modified quantum mechanically. For $N_{f}=N_{c}+1$ the quark multiplets $\left(Q_{f}, \widetilde{Q}_{f}\right)$ have $R_{A F}$ charge equal to $1 / N_{f}$. The mass matrix breaks the $U(1)_{A F}$ symmetry with a charge of $2-2 / N_{f}=2 N_{c} / N_{f}$. It is exactly the charge $U(1)_{A F}$ of equation (XIV.2). On the other hand, the instanton factor $\Lambda^{2 N_{c}-1}$ supplies the right dimensionality. Then, there is the possibility that the classical constraint XIV.2 is modified by nonperturbative contributions to

$$
\operatorname{det} M\left(M^{-1}\right)^{f g}-B^{f} \widetilde{B}^{g}=\Lambda^{2 N_{c}-1} m^{f g} .
$$

On the other hand, one can see that the classical constraints (XIV.1) do not admit modification. Then if $M \neq 0$ we have $B^{f}=\widetilde{B}^{g}=0$. Using (XII.4), we obtain

$$
\operatorname{det} M\left(M^{-1}\right)^{f g}=\Lambda^{2 N_{c}-1} m^{f g},
$$

and the quantum modification (XIV.3) really takes place [36.

\section{B. S-confinement.}

In the massless limit $m^{f g} \rightarrow 0$, (XIV.1) and XIV.2) are satisfied at the quantum level. It means that the origin of field space, $M=B=\widetilde{B}=0$, is an allowed point of the quantum moduli space. On such a point, there is no spontaneous symmetry breaking at all. We use the 't Hooft's anomaly matching conditions to check it. The quantum numbers of the massless fermions at high and low energy are:

\begin{tabular}{c|c|c|c|c|} 
& $S U\left(N_{f}\right)_{L}$ & $S U\left(N_{f}\right)_{R}$ & $U(1)_{B}$ & $U(1)_{A F}$ \\
\hline$\lambda$ & $\mathbf{1}$ & $\mathbf{1}$ & 0 & 1 \\
$\psi_{q}$ & $\mathbf{N}_{\mathbf{f}}$ & $\mathbf{1}$ & 1 & $\frac{1}{N_{f}}-1$ \\
$\psi_{\tilde{q}}$ & $\mathbf{1}$ & $\overline{\mathbf{N}}_{\mathbf{f}}$ & -1 & $\frac{1}{N_{f}}-1$ \\
\hline$\psi_{M}$ & $\mathbf{N}_{\mathbf{f}}$ & $\overline{\mathbf{N}}_{\mathbf{f}}$ & 0 & $\frac{2}{N_{f}}-1$ \\
$\psi_{B}$ & $\overline{\mathbf{N}}_{\mathbf{f}}$ & $\mathbf{1}$ & $N_{f}-1$ & $-\frac{1}{N_{f}}$ \\
$\psi_{\tilde{B}}$ & $\mathbf{1}$ & $\mathbf{N}_{\mathbf{f}}$ & $1-N_{f}$ & $-\frac{1}{N_{f}}$
\end{tabular}

and the anomaly coefficients are:

\begin{tabular}{c|c|c|} 
triangles & high energy & low energy \\
\hline$S U\left(N_{f}\right)^{3}$ & $N_{c} C_{3}$ & $N_{f} C_{3}+\bar{C}_{3}$ \\
\hline$S U\left(N_{f}\right)^{2}$ & $N_{c} T\left(\mathbf{N}_{\mathbf{f}}\right)\left(-\frac{N_{c}}{N_{f}}\right)$ & $N_{f} T\left(\mathbf{N}_{\mathbf{f}}\right)\left(\frac{2}{N_{f}}-1\right)$ \\
$\times U(1)_{A F}$ & & $+T\left(\mathbf{N}_{\mathbf{f}}\right)\left(-\frac{1}{N_{f}}\right)$ \\
\hline$U(1)_{B}^{2} \times U(1)_{A F}$ & $2 N_{c} N_{f}\left(-\frac{N_{c}}{N_{f}}\right)$ & $2 N_{f} N_{c}^{2}\left(-\frac{1}{N_{f}}\right)$ \\
\hline$U(1)_{A F}^{3}$ & $\left(N_{c}^{2}-1\right)$ & $N_{f}^{2}\left(\frac{2}{N_{f}}-1\right)^{3}$ \\
& $+2 N_{f} N_{c}\left(-\frac{N_{c}}{N_{f}}\right)^{3}$ & $+2 N_{f}\left(-\frac{1}{N_{f}}\right)^{3}$ \\
\hline $\operatorname{tr} U(1)_{A F}$ & $\left(N_{c}^{2}-1\right)$ & $N_{f}^{2}\left(\frac{2}{N_{f}}-1\right)$ \\
& $+2 N_{f} N_{c}\left(-\frac{N_{c}}{N_{f}}\right)$ & $+2 N_{f}\left(-\frac{1}{N_{f}}\right)$
\end{tabular}

with complete agreement. Hence, at the origin of field space we have massless mesons and baryons, and the full global symmetry is manifest. It is a singular point, with the number of massless degrees of freedom larger than the dimensionality of the space of vacua. As we move along the moduli space away from the origin, the 'extra' fields become massive and the massless fluctuations match with the dimensionality of the moduli space. As we are in a Higgs/confining phase, there should be a smooth connection of the dynamics at the origin of field space with the one away from it. This dynamics must be given by some nonperturbative superpotential of mesons and baryons. A theory with the previous characteristics is called s-confining.

There is a unique effective superpotential yielding all the constraints [36], 


$$
\mathcal{W}=\frac{1}{\Lambda^{2 N_{f}-3}}\left(\widetilde{B}^{g} M_{g f} B^{f}-\operatorname{det} M\right),
$$

it satisfies:

i) Invariance under all the symmetries.

ii) The equations of motion $\partial \mathcal{W} / \partial M=\partial \mathcal{W} / \partial B=$ $\partial \mathcal{W} / \partial \widetilde{B}=0$ give the constraints XIV.1, XIV.2).

iii) At the origin all the fields are massless.

iv) Adding the bare term $\operatorname{tr}(m M)+b_{f} B^{f}+\widetilde{b}_{f} \widetilde{B}^{f}$ we recover the $N_{f}<N_{c}+1$ results.

\section{SEIBERG'S DUALITY.}

\section{A. The dual SQCD.}

If we try to extend the same view of $S U\left(N_{c}\right)$ SQCD for the case of $N_{f}>N_{c}+1$, i.e., as being in a Higgs/confining phase with the vacuum structure determined by meson and baryons operators satisfying the corresponding classical constraints, to the case of $N_{f}>N_{c}+1$ (it is not possible to modify the classical constraints for $N_{f}>N_{c}+1$ ), we obtain inconsistencies. It is not possible to generate a superpotential yielding to the constraints, and the 't Hooft's anomaly matching conditions are not satisfied. It indicates that for $N_{f}>N_{c}+1$ the Higgs/confining description of SQCD at large distances in terms of just $M, B$ and $\widetilde{B}$ is no longer valid.

For $N_{f}>N_{c}+1$, Seiberg conjectured [38] that the infrared limit of SQCD with $N_{f}$ flavors admits a dual description in terms of an $N=1$ super Yang-Mills gauge theory with $\widetilde{N}_{c}=N_{f}-N_{c}$ number of colors, $N_{f}$ flavors $D^{f}$ and $\widetilde{D}^{f}$ in the fundamental and anti-fundamental representations of $S U\left(N_{f}-N_{c}\right)$ respectively, and $N_{f}^{2}$ gauge singlet chiral superfields $M_{g f}^{(m)}$. The fields $M_{g f}^{(m)}$ couple to $D_{f}$ and $\widetilde{D}_{f}$ through the relevant bare superpotential

$$
\mathcal{W}=M_{g f}^{(m)} \widetilde{D}^{g} D^{f} .
$$

If both theories are going to describe the same physics at large distances, we must be able to give a prescription of the gauge invariant operators $M_{g f}, B^{f_{1} \cdots f_{N_{c}}}$ and $\widetilde{B}^{f_{1} \cdots f_{N_{c}}}$ in terms of the dual microscopic operators $\left(D^{f}, \widetilde{D}^{f}\right)$ and $M_{g f}^{(m)}$. The simplest identification is:

$$
\begin{aligned}
M_{g f} & =\mu M_{g f}^{(m)}, \\
B_{1}^{f_{1} \cdots f_{N_{c}}} & =D^{f_{1}} \cdots D^{f_{N_{c}},} \\
\widetilde{B}^{f_{1} \cdots f_{N_{c}}} & =\widetilde{D}^{f_{1}} \cdots \widetilde{D}^{f_{\widetilde{N}_{c}}} .
\end{aligned}
$$

In the baryon operators the $S U\left(\widetilde{N}_{c}\right)$ color indices of $\left(D^{f}, \widetilde{D}^{f}\right)$ are contracted with the $\widetilde{N}_{c}$ antisymmetric tensor. The scale $\mu$ is introduced because the dimension of the bare operator $M_{g f}^{(m)}$, derived from XV.1 , is one.
This mass scale relates the intrinsic scales $\Lambda$ and $\widetilde{\Lambda}$ of the $S U\left(N_{c}\right)$ and $S U\left(\widetilde{N}_{c}\right)$ gauge theories through the equation

$$
\Lambda^{3 N_{c}-N_{f}} \widetilde{\Lambda}^{3 \widetilde{N}_{c}-N_{f}}=(-1)^{N_{f}-N_{c}} \mu^{N_{f}} .
$$

We see that an strongly coupled $S U\left(N_{c}\right)$ gauge theory corresponds to a weakly coupled $S U\left(\widetilde{N}_{c}\right)$ gauge theory, in analogy with the electric-magnetic duality. From this analogy, we call the $S U\left(N_{c}\right)$ gauge theory the electric one, and the $S U\left(\widetilde{N}_{c}\right)$ gauge theory the magnetic one.

Both theories must have the same global symmetries. The mapping (XV.2) gives the quantum numbers of the magnetic degrees of freedom. Once more, 't Hooft's anomaly matching conditions for the electric and magnetic theories give a non-trivial check of (XV.2). In the following table we write the quantum numbers for the fermions of the magnetic theory:

\begin{tabular}{c|c|c|c|c|} 
& $S U\left(N_{f}\right)_{L}$ & $S U\left(N_{f}\right)_{R}$ & $U(1)_{B}$ & $U(1)_{A F}$ \\
\hline$\tilde{\lambda}$ & $\mathbf{1}$ & $\mathbf{1}$ & 0 & 1 \\
$\psi_{d}$ & $\overline{\mathbf{N}}_{\mathbf{f}}$ & $\mathbf{1}$ & $\frac{N_{c}}{\widetilde{N}_{c}}$ & $\frac{\widetilde{N}_{c}}{N_{f}}$ \\
$\psi_{\tilde{d}}$ & $\mathbf{1}$ & $\mathbf{N}_{\mathbf{f}}$ & $-\frac{N_{c}}{\widetilde{N}_{c}}$ & $\frac{\widetilde{N}_{c}}{N_{f}}$ \\
$\psi_{m}$ & $\mathbf{N}_{\mathbf{f}}$ & $\overline{\mathbf{N}}_{\mathbf{f}}$ & 0 & $1-2 \frac{N_{c}}{N_{f}}$
\end{tabular}

with $\tilde{\lambda}$ the magnetic gluinos. One can check that both theories give the same anomalies.

It can be verified that applying duality again we obtain the original theory.

\section{B. $N_{c}+1<N_{f} \leq 3 N_{c} / 2$. An infrared free non-Abelian Coulomb phase.}

In this range of $N_{f}$ the magnetic theory is not asymptotically free and has a trivial infrared fixed point. At large distances the physical effective degrees of freedom are the fields $D^{f}, D^{f}, M_{g f}$ and the massless super-gluons of the gauge group $S U\left(N_{f}-N_{c}\right)$. At the origin of field space we are in an infrared free non-Abelian Coulomb phase, with a complete screening of its charges in the infrared limit. Observe that the strongly coupled electric theory is weakly coupled in terms of the magnetic degrees of freedom, according to the philosophy of the electric-magnetic duality.

\section{C. $3 N_{c} / 2<N_{f}<3 N_{c}$. An interacting non-Abelian Coulomb phase.}

As in QCD, the $N=1 \mathrm{SQCD}$ has a Banks-Zaks fixed point [39] for $N_{c}, N_{f} \rightarrow \infty$, when $N_{f} / N_{c}=3-\epsilon$ with $\epsilon \ll 1$. We still have asymptotic freedom and under the renormalization group transformations the theory flows 
from the ultraviolet free fixed point to an infrared fixed point with a non-zero finite value of the gauge coupling constant. If there is an interacting superconformal gauge theory the scaling dimensions of some gauge invariant operators should be non-trivial.

The superconformal invariance includes an $R$ symmetry, from which the scaling dimensions of the operators satisfy the lower bound

$$
D \geq \frac{3}{2}|R|
$$

with equality for chiral and anti-chiral operators. The $R$-current is in the same supermultiplet as the energymomentum tensor, whose trace anomaly is zero on the fixed point. It implies that there the $R$-symmetry must be the anomaly-free $U(1)_{A F}$ symmetry. It gives the scaling dimensions of the following chiral operators:

$$
\begin{aligned}
D(M) & =\frac{3}{2} R_{A F}(M)=3 \frac{N_{f}-N_{c}}{N_{f}}, \\
D(B) & =D(\widetilde{B})=\frac{3}{2} \frac{N_{c}\left(N_{f}-N_{c}\right)}{N_{f}} .
\end{aligned}
$$

Unitarity restricts the scaling dimensions of the gauge invariant operators to be $D \geq 1$. If $D=1$, the corresponding operator $\mathcal{O}$ satisfies the free equation of motion $\partial^{2} \mathcal{O}=0$. If $D>1$, there are non-trivial interactions between the operators.

For the range $3 N_{c} / 2<N_{f}<3 N_{c}$, the gauge invariant chiral operators $M, B$ and $\widetilde{B}$ satisfy the unitarity constraint with $D>1$. Seiberg conjectured the existence of such a non-trivial fixed point for any value of $3 N_{c} / 2<N_{f}<3 N_{c}$, at least for large $N_{c}$.

As $\frac{3}{2}\left(N_{f}-N_{c}\right)<N_{f}<3\left(N_{f}-N_{c}\right)$, there is also a non-trivial fixed point in the magnetic theory. Seiberg's claim is that both theories flow to the same infrared fixed point [38].

\section{XVI. $N=2$ SUPERSYMMETRY.}

\section{A. The supersymmetry algebra and its massless representations.}

The $N=2$ supersymmetry algebra, without central charge, is

$$
\begin{aligned}
& \left\{Q_{\alpha}^{(I)}, \bar{Q}_{\dot{\beta}(J)}\right\}=2\left(\sigma^{\mu}\right)_{\alpha \dot{\beta}} P_{\mu} \delta_{J}^{I}, \\
& \left\{Q_{\alpha}^{(I)}, Q_{\beta}^{(J)}\right\}=0
\end{aligned}
$$

with $I, J=1,2$. The algebra XVI.1 has a new symmetry. We can perform unitary rotations of the two supercharges $Q_{\alpha}^{(I)}$ that do leave the anti-commutator relations (XVI.1) invariant. We have an $U(2)_{R}=U(1)_{R} \times S U(2)_{R}$ symmetry. The Abelian factor $U(1)_{R}$ corresponds to the familiar $R$-symmetry of supersymmetric theories that rotate the global phase of the supercharges $Q_{\alpha}^{(I)}$. With respect the $S U(2)_{R}$ group, the supercharges $Q_{\alpha}^{(I)}$ are in the doublet representation 2 .

As in massless $N=1$ supersymmetric representations, half of the supercharges are realized as vanishing operators: $Q_{2}^{(I)}=0$. We normalize the other two supercharges,

$$
a_{1}^{(I)}=\frac{1}{2 \sqrt{E}} Q_{1}^{(I)}
$$

which are an $S U(2)_{R}$ doublet. The massless $N=2$ vector multiplet is a representation constructed from the Clifford vacuum $\mid 1>$, which has helicity $\lambda=1$ and is an $S U(2)_{R}$ singlet. From it we obtain two fermionic states, $\left|1 / 2>^{(I)}=\left(a^{(I)}\right)^{\dagger}\right| 1>$, and a scalar boson $\mid 0>=$ $\left(a^{(1)}\right)^{\dagger}\left(a^{(2)}\right)^{\dagger} \mid 1>$. After CPT doubling we obtain the $N=2$ vector multiplet:

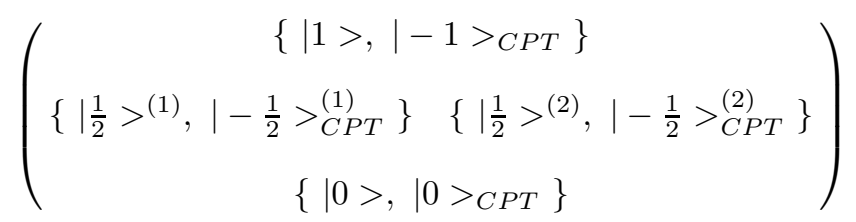

In terms of local fields we have: a vector $A_{\mu}$ (the gauge bosons of some gauge group $G$, since we consider massless representations), which is $S U(2)_{R}$ singlet; two Weyl spinors $\lambda^{(I)}$, the gauginos, arranged in an $S U(2)_{R}$ doublet; and a complex scalar $\phi$, playing the role of the Higgs, a singlet of $S U(2)_{R}$ but in the adjoint of the gauge group $G$. These fields arrange as

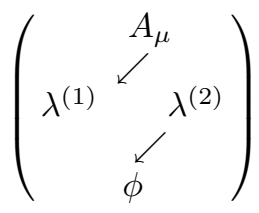

where the arrows indicate the action of the supercharge $\bar{Q}_{\cdot \alpha}^{(1)}$. We can use a manifest $N=1$ supersymmetry representation taking into account that the $N=2$ vector multiplet is composed of an $N=1$ vector multiplet $W_{\alpha}=\left(A_{\mu}, \lambda^{(1)}\right)$ and an $N=1$ chiral multiplet $\Phi=\left(\phi, \lambda^{(2)}\right)$.

The massless $N=2$ hypermultiplet is a representation constructed from a Clifford vacuum $\mid 1 / 2>$, which is an $S U(2)_{R}$ singlet. The action of the two grassmanian operators $a_{\alpha}^{I}$ seems to produce the same particle content that the $N=1$ chiral multiplet, but $|1 / 2>=| 1 / 2, \mathbf{R}>$ is usually in some non-trivial representation $\mathbf{R}$ of a gauge group $G$. As $\mathbf{R} \rightarrow \overline{\mathbf{R}}$ under a $C P T$ transformation, it forces to make the CPT doubling, and the $N=2$ hypermultiplet is built from two $N=1$ chiral multiplets in complex conjugate gauge group representations: 


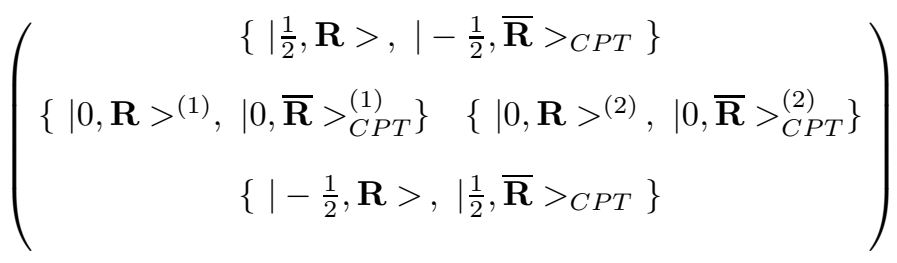

(XVI.5)

Which represents the local fields

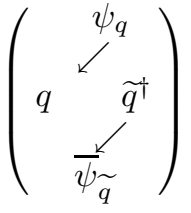

with the complex scalar fields $\left(q, \widetilde{q}^{\dagger}\right)$ in a doublet representation of $S U(2)_{R}$. In terms of $N=1$ superfields we have one chiral superfield $Q=\left(q, \psi_{q}\right)$ in gauge representation $\mathbf{R}$ and another chiral superfield $\widetilde{Q}=(\widetilde{q}, \widetilde{\psi} \widetilde{q})$ in gauge representation $\overline{\mathbf{R}}$. All the field in the hypermultiplet have spin $\leq 1 / 2$. Because of the $C P T$ doubling, the matter content of extended supersymmetry $(N>1)$ is always in vectorial representations of the gauge group.

\section{B. The central charge and massive short representations.}

As shown by Haag, Lapuszanski and Sohnius [40], the $N=2$ supersymmetry algebra admits a central extension:

$$
\begin{aligned}
& \left\{Q_{\alpha}^{a}, Q_{\beta}^{b}\right\}=2 \sqrt{2} \epsilon_{\alpha \beta} \epsilon^{a b} Z, \\
& \left\{\bar{Q}_{\dot{\alpha} a}, \bar{Q}_{\dot{\beta} b}\right\}=2 \sqrt{2} \epsilon_{\dot{\alpha} \dot{\beta}} \epsilon_{a b} \bar{Z} .
\end{aligned}
$$

Since $Z$ commutes with all the generators, we can fix it to be the eigenvalue for the given representation. Now, let us define:

$$
\begin{aligned}
a_{\alpha} & =\frac{1}{2}\left\{Q_{\alpha}^{1}+\epsilon_{\alpha \beta}\left(Q_{\beta}^{2}\right)^{\dagger}\right\}, \\
b_{\alpha} & =\frac{1}{2}\left\{Q_{\alpha}^{1}-\epsilon_{\alpha \beta}\left(Q_{\beta}^{2}\right)^{\dagger}\right\} .
\end{aligned}
$$

Then, in the rest frame, the $N=2$ supersymmetry algebra reduces to

$$
\begin{aligned}
& \left\{a_{\alpha}, a_{\beta}^{\dagger}\right\}=\delta_{\alpha \beta}(\mathcal{M}+\sqrt{2} Z), \\
& \left\{b_{\alpha}, b_{\beta}^{\dagger}\right\}=\delta_{\alpha \beta}(\mathcal{M}-\sqrt{2} Z),
\end{aligned}
$$

with all other anti-commutators vanishing. Since all physical states have positive definite norm, it follows that for massless states, the central charge is trivially realized (i.e., $Z=0$ ), as we used before. For massive states, this leads to a bound on the mass $\mathcal{M} \geq \sqrt{2}|Z|$. When
$\mathcal{M}=\sqrt{2}|Z|$, the operators in (XVI.11) are trivially realized and the algebra resembles the massless case. The dimension of the representation is greatly reduced. For example, a reduced massive $N=2$ multiplet has the same number of states as a massless $N=2$ multiplet. Thus the representations of the $N=2$ algebra with a central charge can be classified as either long multiplets (when $\mathcal{M}>\sqrt{2}|Z|$ ) or short multiplets (when $\mathcal{M}=\sqrt{2}|Z|$ ).

From (XVI.11) it is clear that the BPS states [9.10] (which saturate the bound) are annihilated by half of the supersymmetry generators and thus belong to reduced representations of the supersymmetry algebra. An important consequence of this is that, for BPS states, the relationship between their charges and masses is dictated by supersymmetry and does not receive perturbative or nonperturbative corrections in the quantum theory. This is so because a modification of this relation implies that the states no longer belong to a short multiplet. On the other hand, quantum corrections are not expected to generate the extra degrees of freedom needed to convert a short multiplet into a long multiplet. Since there is no other possibility, we conclude that for short multiplets the relation $\mathcal{M}=\sqrt{2}|Z|$ is not modified either perturbatively or nonperturbatively.

\section{XVII. $N=2 S U(2)$ SUPER YANG-MILLS THEORY IN PERTURBATION THEORY.}

\section{A. The $N=2$ Lagrangian.}

The $N=2$ superspace has two independent chiral spinors $\theta^{(I)}, I=1,2$. The $N=2$ vector multiplet can be written in terms of $N=2$ superspace by the $N=2$ superfield $\Psi\left(x, \theta^{(I)}\right)$ subject to the superspace constraints 41]:

$$
\begin{aligned}
\bar{\nabla}_{\cdot \alpha}^{(I)} \Psi & =0 \\
\nabla_{(I)} \nabla_{(J)} \Psi & =\epsilon_{I K} \epsilon_{J L} \bar{\nabla}^{(K)} \bar{\nabla}^{(L)} \bar{\Psi} .
\end{aligned}
$$

where $\nabla_{(I) \alpha}=D_{(I) \alpha}+\Gamma_{(I) \alpha}$ is the generalized supercovariant derivative of the variable $\theta^{(I)}$, with $\Gamma_{(I) \alpha}$ the superconnection. The $N=1$ superfields are connected to the $N=2$ vector superfield through the equations:

$$
\begin{gathered}
\left.\Psi\right|_{\theta^{(2)}=\bar{\theta}^{(2)}=0}=\Phi\left(x, \theta^{(1)}, \bar{\theta}^{(1)}\right), \\
\left.\nabla_{(2) \alpha} \Psi\right|_{\theta^{(2)}=\bar{\theta}^{(2)}=0}=i \sqrt{2} W_{\alpha}\left(x, \theta^{(1)}, \bar{\theta}^{1}\right) .
\end{gathered}
$$

It results that the renormalizable $N=2$ super YangMills Lagrangian is

$$
\mathcal{L}=\frac{1}{8 \pi} \operatorname{Im}\left(\tau \int d^{2} \theta^{(1)} d^{2} \theta^{(2)} \Psi^{a} \Psi^{a}\right)
$$

with our old friend $\tau=\theta / 2 \pi+i 4 \pi / g^{2}$. In terms of $N=1$ superspace, using XVII.1 and XVII.2), with $\theta \equiv \theta^{(1)}$, the Lagrangian is 


$$
\mathcal{L}=\frac{1}{8 \pi} \operatorname{Im}\left(\tau \int d^{2} \theta W^{\alpha} W_{\alpha}\right)+\frac{1}{g^{2}} \int d^{2} \theta d^{2} \bar{\theta} \Phi^{\dagger} e^{-2 V} \Phi
$$

It looks like $N=1 S U(2)$ gauge theory with an adjoint chiral superfield $\Phi$. The point is that the $1 / g^{2}$ normalization in front of the kinetic term of $\Phi$ gives $N=2$ supersymmetry. In fact, when we perform the remaining superspace integral in (XVII.4), we obtain a Lagrangian that looks like a Georgi-Glashow model with a complex Higgs triplet and the addition of a Dirac spinor $\left(\lambda^{(1)}, \bar{\lambda}^{(2)}\right)$ in the adjoint also. This Lagrangian does not have all the gauge invariant renormalizable terms. $N=2$ supersymmetry restricts the possible terms and gives relations between their couplings, such that at the end there are only the parameters $g^{2}$ and $\theta$.

If we apply perturbation theory to the Lagrangian (XVII.3) we only have to perform a one loop renormalization. This is an indication that in $N=2$ supersymmetry, holomorphy is not lost by radiative corrections. The reason is the following: We expained that the multi-loop renormalization of the coupling $\tau$ came from the generation of non-holomorphic factors $Z\left(\mu / \mu_{0}, g\right)$ in front of the complete $N=1$ superspace integrals. At the level of the Lagrangian (XVII.4), consider the bare coupling $\tau_{0}$ at scale $\mu_{0}$ and integrate out the modes between $\mu_{0}$ and $\mu$. If we consider only the renormalizable terms, $N=1$ supersymmetry gives us

$$
\begin{aligned}
\mathcal{L}_{\text {ren }} & =\frac{1}{8 \pi} \operatorname{Im}\left(\tau(\mu / \Lambda) \int d^{2} \theta W^{\alpha} W_{\alpha}\right) \\
& +Z\left(\frac{\mu}{\mu_{0}}, g_{0}\right) \frac{1}{g^{2}\left(\frac{\mu}{\Lambda}\right)} \int d^{2} \theta d^{2} \bar{\theta} \quad \Phi^{\dagger} e^{-2 V} \Phi
\end{aligned}
$$

where

$$
\tau\left(\frac{\mu}{\Lambda}\right)=\frac{2 i}{\pi} \ln \frac{\mu}{\Lambda}+\sum_{n=0}^{\infty} c_{n}\left(\frac{\Lambda}{\mu}\right)^{4 n}
$$

is the renormalized coupling constant at scale $\mu$. We used the one-loop beta function of $N=2 S U(2)$ gauge theory $b_{0}=4$ and the renormalization group invariant scale $\Lambda \equiv$ $\mu_{0} \exp \left(i \pi \tau_{0} / 2\right)$. The dimensionless constants $c_{n}$ are the coefficients of the $n$-instanton contribution $(\Lambda / \mu)^{4 n}=$ $\exp \left(-8 \pi n / g^{2}(\mu)+i \theta(\mu) n\right)$.

If we compare with the $N=2$ renormalizable Lagrangian (XVII.4 ) we derive that $Z\left(\mu / \mu_{0}, g_{0}\right)=1$. Then, there is no Konishi anomaly and the one-loop renormalization of $\tau$ is all there is in perturbation theory.

\section{B. The flat direction.}

Unlike $N=1$ super Yang-Mills, $N=2$ super YangMills theory includes a complex scalar $\phi$ in the adjoint of the gauge group. This scalar plays the role of a Higgs field through the potential derived from the Lagrangian (XVII.4),

$$
V\left(\phi, \phi^{\dagger}\right)=\frac{1}{2 g^{2}}\left[\phi^{\dagger}, \phi\right]^{2}
$$

The supersymmetric minimum is obtained by the solution of

$$
\left[\phi^{\dagger}, \phi\right]=0
$$

whose solution, up to gauge transformations, is $\phi=a \sigma^{3}$, with $a$ an arbitrary complex number. This is our flat direction. Along it, the $S U(2)$ gauge group is spontaneously broken to the $U(1)$ subgroup. The $\Psi^{ \pm}=$ $\frac{1}{\sqrt{2}}\left(\Psi^{1} \pm i \Psi^{2}\right)$ superfield components have $U(1)$ electric charge $Q_{e}= \pm g$, respectively, and they have the classical squared mass

$$
\mathcal{M}_{W}^{2}=2|a|^{2}
$$

The $\mathcal{A} \equiv \Psi^{3}$ superfield component remains massless. We know that the Lagrangian XVII.3 admits semi-classical dyons with electric charge $Q_{e}=n_{e} g+\theta / 2 \pi$ and magnetic charge $Q_{m}=(4 \pi / g)$, i.e., the points $\left(1, n_{e}\right)$ in the charge lattice. They have the classical squared mass

$$
\mathcal{M}^{2}\left(1, n_{e}\right)=2|a|^{2}\left|n_{e}+\tau\right|^{2} .
$$

Physical masses are gauge invariant. We can use the gauge invariant parametrization of the moduli space in terms of the chiral superfield

$$
U=\operatorname{tr} \Phi^{2}
$$

and traslate the $a$-dependence in previous formulae by an $u$-dependence through the relation $u=\operatorname{tr}\left\langle\phi^{2}\right\rangle$. The classical relation is just $u=a^{2} / 2$.

Then, semi-classical analysis gives $\mathcal{A}$ as the unique light degree of freedom. Only at $u=0$ the full $S U(2)$ gauge symmetry is restored. How is this picture modified by the nonperturbative corrections?. The Seiberg-Witten solution answers this question 13 . 3 .

\section{THE LOW ENERGY EFFECTIVE LAGRANGIAN.}

The $N=2$ vector superfield $\mathcal{A}$ is invariant under the unbroken $U(1)$ gauge transformations. At a scale of the order of the $\mathcal{M}_{W}$ mass, i.e., of the order or $|u|^{1 / 2}$, the most general $N=2$ Wilsonian Lagrangian, with two derivatives and four fermions terms, that can be constructed from the light degrees of freedom in $\mathcal{A}$ is

\footnotetext{
${ }^{\S}$ Some additional reviews on the Seiberg-Witten solution are 42 .
} 


$$
\mathcal{L}_{\text {eff }}=\frac{1}{4 \pi} \operatorname{Im}\left(\int d^{2} \theta^{(1)} d^{2} \theta^{(2)} \mathcal{F}(\mathcal{A})\right)
$$

with $\mathcal{F}$ a holomorphic function of $\mathcal{A}$, called the prepotential. We stress that the unique inputs to equation (XVIII.1) are $N=2$ supersymmetry and that $\mathcal{A}$ is a vector multiplet. We derive an immediate consequence of the general form of the effective Lagrangian (XVIII.1): $N=2$ supersymmetry prevents the generation of a superpotential for the $N=1$ chiral superfield of $\mathcal{A}$. It means that the previously derived flat direction, parametrized by the arbitrary value $u=\operatorname{tr}\left\langle\phi^{2}\right\rangle$, is not lifted by nonperturbative corrections.

In terms of $N=1$ superspace we have

$$
\begin{aligned}
\mathcal{L}_{\text {eff }} & =\frac{1}{4 \pi} \operatorname{Im}\left(\int d^{2} \theta \frac{1}{2} \tau(A) W^{\alpha} W_{\alpha}\right) \\
& +\int d^{2} \theta d^{2} \bar{\theta} K(A, \bar{A})
\end{aligned}
$$

where

$$
\begin{aligned}
\tau(A) & =\frac{\partial^{2} \mathcal{F}}{\partial A^{2}}(A), \\
K(A, \bar{A}) & =\operatorname{Im}\left(\frac{\partial \mathcal{F}}{\partial A} \bar{A}\right),
\end{aligned}
$$

and $A$ is the $N=1$ chiral multiplet of $\mathcal{A}$.

The Wilsonian Lagrangian (XVIII.2) is an Abelian gauge theory defined at some scale of order $\mathcal{M}_{W} \sim$ $|u|^{1 / 2}$. Interaction terms come out after the expansion $A=a+\hat{A}$, with $a$ the vacuum expectation value of the Higgs field, and $\hat{A}$ the quantum fluctuations of the chiral superfield. The matching at scale $|u|^{1 / 2}$ with the high energy $S U(2)$ theory is performed by the renormalization group:

$$
\tau(u)=\frac{i}{\pi} \ln \frac{u}{\Lambda^{2}}+\sum_{n=0}^{\infty} c_{n}\left(\frac{\Lambda^{2}}{u}\right)^{2 n} .
$$

Observe that the phase of the dimensionless quotient $u / \Lambda^{2}$ plays the role of the bare $\theta_{0}$ angle. If we are able to know the relation between the $u$ and $a$ variables, i.e., the function $u(a)$, we can replace it into XVIII.5) to obtain $\tau(a)$. Integrating twice in the variable $a$ we obtain the prepotential

$$
\mathcal{F}(a)=\frac{i}{2 \pi} a^{2} \ln \frac{a^{2}}{\Lambda^{2}}+a^{2} \sum_{n=1}^{\infty} \mathcal{F}_{k}\left(\frac{\Lambda}{a}\right)^{4 k} .
$$

If we look at the terms of the Lagrangian (XVIII.2) proportional to the dimensionless constant $\mathcal{F}_{n}$, they correspond to the effective interaction terms created by the $n$-instanton contribution, as expected. For $a \rightarrow \infty$, the instanton contributions go to zero. This is an expected result, since at $a \rightarrow \infty$ the matching takes place at weak coupling due to asymptotic freedom. In this region perturbation theory is applicable and we can believe the semi-classical relation, $u \sim a^{2} / 2$.

\section{BPS BOUND AND DUALITY.}

The $N=2$ supersymmetry algebra gives the mass bound

$$
\mathcal{M} \geq \sqrt{2}|Z|,
$$

with $Z$ the central charge. The origin of the central charge is easy to understand: the supersymmetry charges $Q$ and $\bar{Q}$ are space integrals of local expressions in the fields (the time component of the super-currents). In calculating their anti-commutators, one encounters surface terms which are normally neglected. However, in the presence of electric and magnetic charges, these surface terms are non-zero and give rise to a central charge. When one calculates the central charge that arises from the classical Lagrangian (XVII.3) one obtains 43]

$$
Z=a e(n+m \tau)
$$

so that $\mathcal{M} \geq \sqrt{2}|Z|$ coincides with the Bogomol'nyi bound (VI.12).

But the equation (XIX.2) is a classical result. The effective Lagrangian (XVIII.1) includes all the nonperturbative quantum corrections of the higher modes. To get their contribution to the BPS bound, we just have to compute the central charge that is derived from the effective Lagrangian (XVIII.1). The result is

$$
Z\left(n_{m}, n_{e}\right)=n_{e} a+n_{m} a_{D},
$$

for a supermultiplet located in the charge lattice at $\left(n_{m}, n_{e}\right)$. We have defined the $a_{D}$ function

$$
a_{D} \equiv \frac{\partial \mathcal{F}}{\partial a}(a)
$$

This function plays a crucial role in duality. Observe that under the $S L(2, \mathbf{Z})$ transformation $M=\left(\begin{array}{ll}\alpha & \beta \\ \gamma & \delta\end{array}\right)$ of the charge lattice,

$$
\left(n_{m}, n_{e}\right) \rightarrow\left(n_{m}, n_{e}\right) M^{-1},
$$

the invariance of the central charge demands

$$
\left(\begin{array}{c}
a_{D} \\
a
\end{array}\right) \rightarrow M\left(\begin{array}{c}
a_{D} \\
a
\end{array}\right) .
$$

Its action on the effective gauge coupling $\tau=\partial a_{D} / \partial a$ is

$$
\tau \rightarrow \frac{\alpha \tau+\beta}{\gamma \tau+\delta} .
$$

The $S$-transformation, that interchanges electric with magnetic charges, makes

$$
\begin{gathered}
a_{D} \rightarrow a, \\
a \rightarrow-a_{D} .
\end{gathered}
$$


Then, $a_{D}$ is the dual scalar photon, that couples locally with the monopole $(1,0)$ through the dual gauge coupling $\tau_{D}=-1 / \tau$.

From (XVIII.3) and (XVIII.4), we see that $\operatorname{Im} \tau(a)$ is the Kähler metric of the Kähler potential $K(a, \bar{a})$,

$$
d^{2} s=[\operatorname{Im} \tau(a)] d a d \bar{a} .
$$

Physical constraints demands the metric be positive definite, $\operatorname{Im} \tau>0$. However, if $\tau(a)$ is globally defined the metric cannot be positive definite as the harmonic function $\operatorname{Im} \tau(a)$ cannot have a minimum. This indicates that the above description of the metric in terms of the variable $a$ must be valid only locally. In the weak coupling region, $|u| \gg|\Lambda|$, where $\tau(a) \sim(2 i / \pi) \ln (a / \Lambda)$, we have that $\operatorname{Im} \tau(a)>0$, but for $a \sim \Lambda$, when the theory is at strong coupling and the nonperturbative effects become important, the perturbative result does not give the correct physical answer. Two things should happen: the instanton corrections must secure the positivity of the metric and physics must be described in terms of a new local variable $a^{\prime}$. Which is this new local variable? If we do not want to change the physics, the change of variables must be an isometry of the Kähler metric (XIX.9). In terms of the variables $\left(a_{D}, a\right)$ the Kähler metric is

$$
d^{2} s=\operatorname{Im}\left(d a_{D} d \bar{a}\right)=-\frac{i}{2}\left(d a_{D} d \bar{a}-d a d \bar{a}_{D}\right),
$$

The complete isometry group of (XIX.10) is $\left(\begin{array}{c}a_{D} \\ a\end{array}\right) \rightarrow$ $M\left(\begin{array}{c}a_{D} \\ a\end{array}\right)+\left(\begin{array}{c}p \\ q\end{array}\right)$ with $M \in S L(2, \mathbf{R})$ and $p, q \in \mathbf{R}$. But the invariance of the central charge puts $p=q=0$ **** and the Dirac quantization condition restricts $M \in$ $S L(2, \mathbf{Z})$. We arrive to an important result: in some region of the moduli space we have to perform an electricmagnetic duality transformation.

\section{SINGULARITIES IN THE MODULI SPACE.}

As $\operatorname{Im} \tau$ cannot be globally defined on the $u$ plane, there must be some singularities $u_{i}$ indicating the multivaluedness of $\tau(u)$. If we perform a loop arround a singularity $u_{i}$, there is a non-trivial monodromy action $M_{i}$ on $\tau(u)$. This action should be an isometry of the Kähler metric, if we do not want to change the physics. It implies that the monodromies $M_{i}$ are elements of the $S L(2, \mathbf{Z})$ group.

In fact, we have found already one non-trivial monodromy because of the perturbative contributions. The multivalued logarithmic dependence of $\tau$ gives the monodromy. For $u \sim \infty, \tau \sim(i / \pi) \ln \left(u / \Lambda^{2}\right)$. In that region, the loop $u \rightarrow e^{2 \pi i} u$ applied on $\tau(u)$ gives

\footnotetext{
${ }^{* * *}$ In $N=2$ SQCD with massive matter, the central charge allows to have $p, q \neq 0$ [44].
}

$$
\tau \rightarrow \tau-2
$$

Its associated monodromy is

$$
M_{\infty}=\left(\begin{array}{cc}
-1 & 2 \\
0 & -1
\end{array}\right)=P T^{-2}
$$

which acts on the variables $\left(a_{D}, a\right)$ as

$$
\begin{array}{r}
a_{D} \rightarrow-a_{D}+2 a, \\
a \rightarrow-a .
\end{array}
$$

As it should be, the monodromy is a symmetry of the theory. $T^{-2}$ just shifts the $\theta$ parameter by $-4 \pi$, and $P$ is the action of the Weyl subgroup of the $S U(2)$ gauge group. Then, the monodromy at infinity $M_{\infty}$ leaves the $a$ variable invariant (up to a gauge transformation).

The monodromy at infinity means there must be some singularity in the $u$ plane. How many singularities?. We know that the anomalous $U(1)_{R}$ symmetry is broken by instantons, and that there is an unbroken $\mathbf{Z}_{8}$ subgroup because the one-instanton sector has eight fermionic zero modes. The $U=\operatorname{tr} \Phi^{2}$ operator has $R$-charge four. It means that the $u \rightarrow-u$ symmetry is spontaneously broken, leading to equivalent physical vacua. Then, if $u_{0}$ is a singular point, $-u_{0}$ must be also another singular point.

Let us assume that there is only one singularity. If this were the situation, the monodromy group would be Abelian, generated only by the monodromy at infinity. From the monodromy invariance of the variable $a$ under $M_{\infty}$, we would have that $a$ is a good variable to describe the physics of the whole moduli space. This is in contradiction with the holomorphy of $\tau(a)$.

Seiberg and Witten made the assumption that there are only two singularities, which they normalized to be $u_{1}=\Lambda^{2}$ and $u_{2}=-\Lambda^{2}$. This assumption leads to a unique and elegant solution that passes many tests.

\section{THE PHYSICAL INTERPRETATION OF THE SINGULARITIES.}

The most natural physical interpretation of singularities in the $u$ plane is that some additional massless particles appear at the singular point $u=u_{0}$.

The particles will arrange in some $N=2$ supermultiplet and will be labeled by some quantum numbers $\left(n_{m}, n_{e}\right)$. If the massless particle is purely electric, the Bogomol'nyi bound implies $a\left(u_{0}\right)=0$. It would mean that the $\mathrm{W}$-bosons become massless at $u_{0}$ and the whole $S U(2)$ gauge symmetry is restored there. It would imply the existence of a non-Abelian infrared fixed point with $\left\langle\operatorname{tr} \phi^{2}\right\rangle \neq 0$. By conformal invariance, the scaling dimension of the operator $\operatorname{tr} \phi^{2}$ at this infrared fixed point would have to be zero, i.e., it would have to be the identity operator. It is not possible since $\operatorname{tr} \phi^{2}$ is odd under a global symmetry.

Then, the particles that become massless at the singular point $u_{0}$ are arranged in an $N=2$ supermultiplet 
of $\operatorname{spin} \leq 1 / 2$. The possibilities are severely restricted by the structure of $N=2$ supersymmetry: the multiplet must be an hypermultiplet that saturates the BPS bound. As we have derived that we should have $a \neq 0$ for all the points of the moduli space, the singular BPS state must have a non-zero magnetic charge.

Near its associated singularity, the light $N=2$ hypermultiplet is a relevant degree of freedom to be considered in the low energy Lagrangian. The coupling to the massless photon of the unbroken $U(1)$ gauge symmetry has to be local. Therefore, we apply a duality transformation to describe the relevant degree of freedom $\left(n_{m}, n_{e}\right)$ as a purely electric state $(0,1)$,

$$
(0,1)=\left(n_{m}, n_{e}\right) N^{-1},
$$

with $N$ the appropiate $S L(2, \mathbf{Z})$ transformation. The dual variables are the good local variables near the $u_{0}$ singularity. It implies that the monodromy matrix must leave invariant the singular state $\left(n_{m}, n_{e}\right)$. This constraint plus the $U(1) \beta$-function give the monodromy matrix

$$
M\left(n_{m}, n_{e}\right)=\left(\begin{array}{cc}
1+2 n_{m} n_{e} & 2 n_{e}^{2} \\
-2 n_{m}^{2} & 1-2 n_{e} n_{m}
\end{array}\right)
$$

In fact, in terms of the local variables,

$$
\left(\begin{array}{c}
a_{D}^{\prime} \\
a^{\prime}
\end{array}\right)=N\left(\begin{array}{c}
a_{D} \\
a
\end{array}\right)
$$

the monodromy matrix is just $T^{2}$. This result can be understood as follows: The renormalizable part of the low energy Lagrangian is just $N=2$ QED with one light hypermultiplet with mass $\sqrt{2}\left|a^{\prime}\right|=\sqrt{2}\left|n_{m} a_{D}+n_{e} a\right|$. It has a trivial infrared fixed point, and the theory is weakly coupled at large distances. Perturbation theory gives

$$
\tau^{\prime} \simeq-\frac{i}{\pi} \ln a^{\prime} .
$$

On the other hand, by the monodormy invariance of $a^{\prime}$, we have $a^{\prime}(u) \simeq c_{0}\left(u-u_{0}\right)$, this gives the monodromy matrix $T^{2}: \tau^{\prime} \rightarrow \tau^{\prime}+2$.

With all the monodromies taken in the counter clockwise direction, and the monodromy base point chosen in the negative imaginary part of the complex $u$ plane, we have the topological constraint

$$
M_{-\Lambda^{2}} M_{\Lambda^{2}}=M_{\infty} .
$$

If we use the expression XXI.2 for the monodromies $M_{ \pm \Lambda^{2}}$ and that $M_{\infty}=P T^{-2},(\mathrm{XXI} .5)$ implies that the magnetic charge of the singular states must be \pm 1 . Then, they exist semi-classically and are continuousy connected with the weak coupling region. Moreover, if the state $\left(1, n_{e}\right)$ becomes massless at $u=\Lambda^{2}$, then (XXI.5) gives the massless state $\left(1, n_{e}-1\right)$ at $u=-\Lambda^{2}$. It is consistent with the action of the spontaneously broken symmetry $u \rightarrow-u$, since by the expression of $\tau(u)$ in (XVIII.5) we have that $\theta_{\text {eff }}\left(-\Lambda^{2}\right)=2 \pi \operatorname{Re}\left(\tau\left(-\Lambda^{2}\right)\right)=2 \pi$, and by the Witten effect gives the same physical electric charge to the massless states at $u= \pm \Lambda^{2}$.

Seiberg and Witten took the simplest solution: a purely magnetic monopole $(1,0)$ 们 becomes massless at $u=\Lambda^{2}$. With our chosen monodromy base point, the state with quantum numbers $(1,-1)$ has vanishing mass at $u=-\Lambda^{2}$.

\section{THE SEIBERG-WITTEN SOLUTION.}

\section{A. The inputs.}

After this long preparation, we can present the solution of the model. The moduli space is the compactified $u$-plane punctured at $u=\Lambda^{2},-\Lambda^{2}, \infty$. These singular points generate the monodromies:

$$
\begin{gathered}
M_{\Lambda^{2}}=\left(\begin{array}{cc}
1 & 0 \\
-2 & 1
\end{array}\right), \\
M_{-\Lambda^{2}}=\left(\begin{array}{ll}
-1 & 2 \\
-2 & 3
\end{array}\right), \\
M_{\infty}=\left(\begin{array}{cc}
-1 & 2 \\
0 & -1
\end{array}\right),
\end{gathered}
$$

which act on the holomorphic function $\tau(u)$ by the corresponding modular transformations. Physically, the function $\tau(u)$ is the effective coupling at the vacuum $u$ and its asymptotic behavior near the punctured points $u=\Lambda^{2},-\Lambda^{2}, \infty$, is known.

\section{B. The geometrical picture.}

A torus is a two dimensional compact Riemann surface of genus one. Topologically it can be described by a two dimensional lattice with complex periods $\omega$ and $\omega_{D}$. The construction is the following: a point $z$ in the complex plane is identifyed with the points $z+\omega$ and $z+\omega_{D}$ (with the convention $\left.\operatorname{Im}\left(\omega_{D} / \omega\right)>0\right)$, to get the topology of a torus. Then, the $S L(2, \mathbf{Z})$ transformations

$$
\left(\begin{array}{c}
\omega_{D} \\
\omega
\end{array}\right) \rightarrow M\left(\begin{array}{c}
\omega_{D} \\
\omega
\end{array}\right)
$$

leave invariant the torus. If we rescale the lattice with $1 / \omega$, the torus is characterized just by the modulus

$$
\tau \equiv \frac{\omega_{D}}{\omega}
$$

\footnotetext{
${ }^{\dagger \dagger}$ Observe that by Witten effect, the shift $\theta \rightarrow \theta+2 \pi n$ transforms $(1,0) \rightarrow(1, n)$. There is a complete democracy between the semi-classical stable dyons.
} 
up to $S L(2, \mathbf{Z})$ transformations,

$$
\tau \sim \frac{\alpha \tau+\beta}{\gamma \tau+\delta}
$$

Algebraically the torus can be described by a complex elliptic curve

$$
y^{2}=4\left(x-e_{1}\right)\left(x-e_{2}\right)\left(x-e_{3}\right) .
$$

The toric structure arises because of the two Riemman sheets in the $x$ plane joined through the two branch cuts going from $e_{1}$ to $e_{2}$ and $e_{3}$ to infinity (see fig. 2).
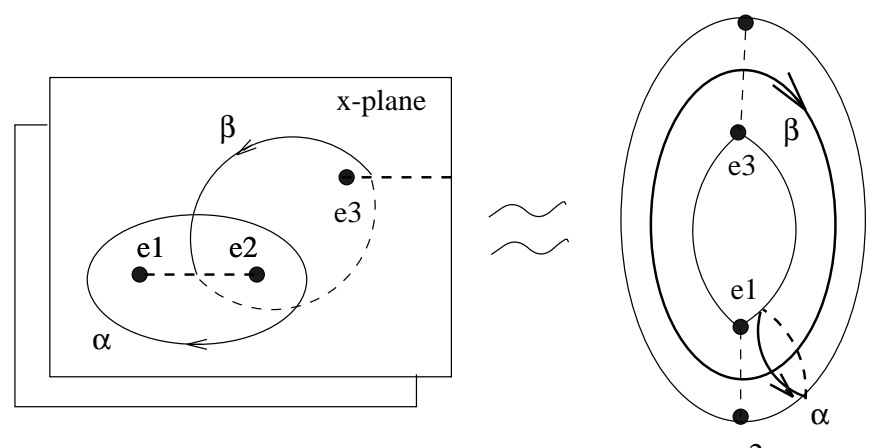

FIG. 2. The elliptic curve (XXII.3) gives the topology of a torus.

The lattice periods are obtained by integrating the Abelian differential of first kind $d x / y$ along the two homologically non-trivial one-cycles $\alpha$ and $\beta$, with intersection number $\beta \cdot \alpha=1$,

$$
\begin{aligned}
\omega_{D} & =\oint_{\beta} \frac{d x}{y}, \\
\omega & =\oint_{\alpha} \frac{d x}{y} .
\end{aligned}
$$

They have the property that $\operatorname{Im} \tau>0$.

\section{The Physical connection with $N=2$ super Yang-Mills.}

The breakthough of Seiberg and Witten for the solution of the model was the identification of the complex effective coupling $\tau(u)$ at a given vacuum $u$ with the modulus of a $u$-dependent torus. At any point $u$ of the moduli space, they associated an elliptic curve

$$
y^{2}=4 \prod_{i=1}^{3}\left(x-e_{i}(u)\right)
$$

with its lattice periods given by (XXII.4.

The identification of the physical coupling $\tau(u)=$ $\partial a_{D} / \partial a$ with the modulus $\tau_{u}=\omega_{D}(u) / \omega(u)$ of the elliptic curve (XXII.5),

$$
\tau(u)=\frac{\partial a_{D} / \partial u}{\partial a / \partial u}=\frac{\oint_{\beta} d x / y}{\oint_{\alpha} d x / y}=\tau_{u},
$$

leads to the formulae:

$$
\begin{array}{r}
a_{D}=\oint_{\beta} \lambda(u), \\
a=\oint_{\alpha} \lambda(u),
\end{array}
$$

where $\lambda(u)$ is an Abelian differential with the property that

$$
\frac{\partial \lambda}{\partial u}=f(u) \frac{d x}{y}+d g .
$$

Then, the solution of the problem is reduced to finding the family of elliptic curves (XXII.5) and the holomorphic function $f(u)$. The conditions at the begining of this section fix a unique solution. The family of elliptic curves is determined by the monodromy group generated by the monodromy matrices. The matrices (XXII.1) generate the group $\Gamma(2)$, the subgroup of $S L(2, \mathbf{Z})$ consisting of matrices congruent to the identity modulo 2 . It gives the elliptic curves

$$
y^{2}=\left(x^{2}-\Lambda^{4}\right)(x-u) .
$$

Finally, the function $f(u)$ is determined by the asymptotic behavior of $\left(a_{D}, a\right)$ at the singular points. The answer is $f=-\sqrt{2} / 4 \pi$.

\section{BREAKING $N=2$ TO $N=1$. MONOPOLE CONDENSATION AND CONFINEMENT.}

In this section we will exhibit an explicit realization of the confinement mechanism envisaged by Mandelstam 45] and 't Hooft's through the condensation of light monopoles.

In the $N=2$ model, we have found points in the moduli space where the relevant light degrees of freedom are magnetic particles. Since we have the exact solution of the low energy $N=2$ model, it would be nice to answer in which phase the dynamics of the model, or controlable deformations of it, locates the vacuum.

For the $N=2$ model we already know from section XVIII that $N=2$ supersymmetry does not allow the generation of a superpotential just for the $N=1$ chiral multiplet of the $N=2$ vector multiplet. It means that the theory is always in an Abelian Coulomb phase. The exact solution of the model allowed us to know which are all the instanton corrections to the low energy Lagrangian. Remarkably enough, the instanton series admits a resumation in terms of magnetic variables.

To go out of the Coulomb branch, we need a superpotential for the chiral superfield $\Phi$. In [13] an explicit 
mass term for the chiral superfield was added in the bare Lagrangian,

$$
\mathcal{W}_{\text {tree }}=m \operatorname{tr} \Phi^{2} .
$$

It breaks $N=2$ to $N=1$ supersymmetry. At low energy, we will have an effective superpotential $\mathcal{W}\left(m, M, \widetilde{M}, A_{D}\right)$. Once again, holomorphy of the superpotential and selection rules from the symmeries will fix the exact form of $\mathcal{W}$. In terms of $N=1$ superspace, only the subgroup $U(1)_{J} \subset S U(2)_{R}$ is manifestly a symmetry. It is a non-anomalous $R$-symmetry (rotates the complex phases of $\theta^{(I)}, I=1,2$, in opposite directions.). The corresponding charge of $\Phi$ is zero. As superpotentials should have charge two, from (XXIII.1) we derive that the parameter $m \neq 0$ breaks the $U(1)_{J}$ symmetry by two units. On the other hand, the $N=1$ chiral superfields $M$ and $\widetilde{M}$ are in an $N=2$ hypermultiplet and therefore, both have charge one. Imposing that $\mathcal{W}$ is a regular function at $m=\widetilde{M} M=0$, we find that it is of the form $\mathcal{W}=m f_{1}\left(A_{D}\right)+\widetilde{M} M f_{2}\left(A_{D}\right)$. For $m \rightarrow 0$, the effective superpotential flows to the tree level superpotential (XXIII.1) plus the term $\sqrt{2} A_{D} \widetilde{M} M$. As the functions $f_{1}$ and $f_{2}$ are independent of $m$, we obtain the exact result

$$
\mathcal{W}=\sqrt{2} A_{D} \widetilde{M} M+m U\left(A_{D}\right) .
$$

We found what we were looking for: an exact effective superpotential with a term which depends only of the $N=1$ chiral composite operator $U$. It presumely will remove the flat direction. The $N=2$ to $N=1$ breaking makes no loger valid the hiden $N=2$ holomorphy in the Kähler potential $K(A, \bar{A})$. But as long as there is an unbroken supersymmetry, the vacuum configuration corresponds to the solution of the equations

$$
\begin{aligned}
d \mathcal{W} & =0, \\
D=|M|^{2}-|\widetilde{M}|^{2} & =0 .
\end{aligned}
$$

From the exact solution we know that $d u / d a_{D} \neq 0$ at $a_{D}=0$. Thus (up to gauge transformations)

$$
\begin{aligned}
M & =\widetilde{M}=\left(-m u^{\prime}(0) / \sqrt{2}\right)^{1 / 2}, \\
a_{D} & =0 .
\end{aligned}
$$

Expanding around this vacuum we find:

i) There is a mass gap of the order $(m \Lambda)^{1 / 2}$.

ii) The objects that condense are magnetic monopoles. There are electric flux tubes with a non-zero string tension of the order of the mass gap, that confines the electric charges of the $U(1)$ gauge group.

The spontaneously broken symmetry $u \rightarrow-u$ carries the theory to the 'dyon region', with the local variable $a_{D}-a$. The perturbing superpotential there, $m U\left(a_{D}-\right.$ $a)$, also produces the condensation of the 'dyon' with physical electric charge zero at the point $a_{D}-a=0$. Then, we have two physically equivalent vacua, related by an spontaneously broken symmetry, in agreement with the Witten index of $N=1 S U(2)$ gauge theory.

\section{BREAKING $N=2$ TO $N=0$.}

When the $N=2$ theory is broken to the $N=1$ theory through the decoupling of the chiral superfield $\Phi$ in the adjoint, we have seen that the mechanism of confinement takes place because of the condensation of a magnetic monopole. The natural question is if this results can be extended to non supersymmetric gauge theories.

The $N=1,2$ results were based on the use of holomorphy; the question is whether the properties connected with holomorphy can be extended to the $N=0$ case. The answer is positive provided supersymmetry is broken via soft breaking terms.

The method is to promote some couplings in the supersymmetric Lagrangian to the quality of frozen superfields, called spurion superfields. We could think they correspond to some heavy degrees of freedom which at low energies have been decoupled. Their trace is only through their vacuum expectation values appearing in the Lagrangian and are parametrized by the spurion superfields 46].

In the $N=2$ theory we will promote some couplings to the status of spurion superfields. The property of holomorphy in the prepotential will be secured if the introduced spurions are $N=2$ vector superfields 14, 15.

In the bare Lagrangian of the $N=2 S U(2)$ gauge theory (XVII.3), there is only one parameter: $\tau_{0}$. The $N=2$ softly broken theory is obtained by the bare prepotential

$$
\mathcal{F}_{0}=\frac{1}{\pi} \mathcal{S} \mathcal{A}^{a} \mathcal{A}^{a},
$$

where $S$ is an dimensionless $N=2$ vector multiplet whose scalar component gives the bare coupling constant, $s=\frac{\pi}{2} \tau_{0}$. The factor of proporcionality is related with the one loop coefficient of the beta function, such that $\Lambda=\mu_{0} \exp (i s)$. Inspired by String Theory, we call $S$ the dilaton spurion. The source of soft breaking comes from the non vanishing auxiliary fields, $F_{0}$ and $D_{0}$, in the dilaton spurion $S$.

The tree level mass terms arising from the softly broken bare Lagrangian (XXIV.1) are the following: the Wbosons get a mass term by the usual Higgs mechanism, with the mass square equal to $2|a|^{2}$; the photon of the unbroken $U(1)$ remains massless; the gauginos get a mass square $\mathcal{M}_{1 / 2}^{2}=\left(\left|F_{0}\right|^{2}+D_{0}^{2} / 2\right)(4 \operatorname{Im} s)^{-1}$; all the scalar components, except the real part of $\phi^{3}$ which do not have a bare mass term, get a square mass $\mathcal{M}_{0}^{2}=4 \mathcal{M}_{1 / 2}^{2}$.

At low energy, i.e., at scales of the order $|u|^{1 / 2} \sim \Lambda$, the Wilsonian effective Lagrangian up to two derivatives and four fermions terms is given by the effective prepotential $\mathcal{F}(a, \Lambda)$ found in the $N=2$ model, but with the difference that the bare coupling constant is replaced by the

\footnotetext{
${ }^{\ddagger}$ Soft breaking of $N=1 \mathrm{SQCD}$ has been studied in 47$]$.
} 
dilaton spurion, i.e., $\Lambda \rightarrow \mu_{0} \exp (i \mathcal{S})$. Then, the prepotential depends on two vector multiplets and the effective Lagrangian becomes

$$
\begin{aligned}
\mathcal{L} & =\frac{1}{4 \pi} \operatorname{Im}\left(\int d^{4} \theta \frac{\partial \mathcal{F}}{\partial A^{i}} \bar{A}^{i}+\int d^{2} \theta \frac{1}{2} \frac{\partial^{2} \mathcal{F}}{\partial A^{i} \partial A^{j}} W^{i} W^{j}\right) \\
& +\mathcal{L}_{H M} .
\end{aligned}
$$

with $A^{i}=(S, A)$ and $\mathcal{L}_{H M}$ the $N=2$ Lagrangian that includes the monopole hypermultiplet. Observe that the dilaton spurion do not enter in the Lagrangian of the hypermultiplets, in agreement with the $N=2$ nonrenormalization theorem of [27]. The low energy couplings are determined by the $2 \times 2$ matrix

$$
\tau_{i j}(a, s)=\frac{\partial^{2} \mathcal{F}}{\partial a^{i} \partial a^{j}} .
$$

The supersymmetry breaking generates a non-trivial effective potential for the scalar fields,

$$
\begin{aligned}
V_{e f f} & =\left(b_{00}-\frac{b_{01}^{2}}{b_{11}}\right)\left(\left|F_{0}\right|^{2}+\frac{1}{2} D_{0}^{2}\right) \\
& +\frac{b_{01}}{b_{11}}\left[\sqrt{2}\left(F_{0} m \tilde{m}+\bar{F}_{0} \bar{m} \bar{m}\right)+D_{0}\left(|m|^{2}-|\widetilde{m}|^{2}\right)\right] \\
& +\frac{1}{2 b_{11}}\left(|m|^{2}+|\widetilde{m}|^{2}\right)^{2}+2|a|^{2}\left(|m|^{2}+|\widetilde{m}|^{2}\right), \quad(\mathrm{XX}
\end{aligned}
$$

where we have defined $b_{i j}=(4 \pi)^{-1} \operatorname{Im} \tau_{i j} . m$ and $\tilde{m}$ are the scalar components of the chiral superfields $M$ and $\widetilde{M}$ of the monopole hypermultiplet, respectively. Observe that the first line of (XXIV.4 is independent of the monopole degrees of freedom. To be sure that such quantity gives the right amount of energy at any point of the moduli space, where different local descriptions of the physics are necessary, it must be duality invariant. This is the case for any $S L(2, \mathbf{Z})$ transformation.

The auxiliary fields of the dilaton spurion are in the adjoint representation of the group $S U(2)_{R}$ and have $U(1)_{R}$ charge two. We can consider the situation of $D_{0}=0, F_{0}=f_{0}>0$ without any loss of generality, since it is related with the case of $D_{0} \neq 0$ and complex $F_{0}$ just by the appropiate $S U(2)_{R}$ rotation.

We have to be careful with the validity of our approximations. Because of supersymmetry, the expansion in derivatives is linked with the expansion in fermions and the expansion in auxiliary fields. The exact solution of Seiberg and Witten is only for the first terms in the derivative expansion of the effective Lagrangian, in particular up to two derivatives. At the level of the softly broken effective Lagrangian, the exact solution of Seiberg and Witten only gives us the terms at most quadratic in the supersymmetry breaking parameter $f_{0}$. The expansion is performed in the dimensionless parameter $f_{0} / \Lambda$. Our ignorance on the higher derivative terms of the effective Lagrangian is traslated into our ignorance the terms of $\mathcal{O}\left(\left(f_{0} / \Lambda\right)^{4}\right)$. Hence our results are reliable for small values of $f_{0} / \Lambda$, and this is far from the supersymmetry decoupling limit $f_{0} / \Lambda \rightarrow \infty$.
But for moderate values of the supersymmetry breaking parameter, the effective Lagrangian (XXIV.2) gives the large distance physics of a non-supersymmetric gauge theory at strong coupling. If we minimize the effective potential XXIV.4 with respect to the monopoles, we obtain the energy of the vacuum $u$

$$
\begin{aligned}
V_{e f f}(u) & =\left(b_{00}(u)-\frac{b_{01}^{2}(u)}{b_{11}(u)}\right)\left|F_{0}\right|^{2} \\
& -\frac{2}{b_{11}(u)} \rho^{4}(u)
\end{aligned}
$$

where $\rho(u)$ is a positive function that gives the monopole condensate at $u$

$$
\begin{aligned}
& |m|^{2}=|\widetilde{m}|^{2}=\rho^{2}(u)=\frac{\left|b_{01}\right| f_{0}}{\sqrt{2}}-b_{11}|a|^{2}>0 \quad \text { (XXIV.6) } \\
& \text { or } m=\widetilde{m}=\rho(u)=0 \text { if }\left|b_{01}\right| f_{0}<\sqrt{2} b_{11}|a|^{2} .
\end{aligned}
$$

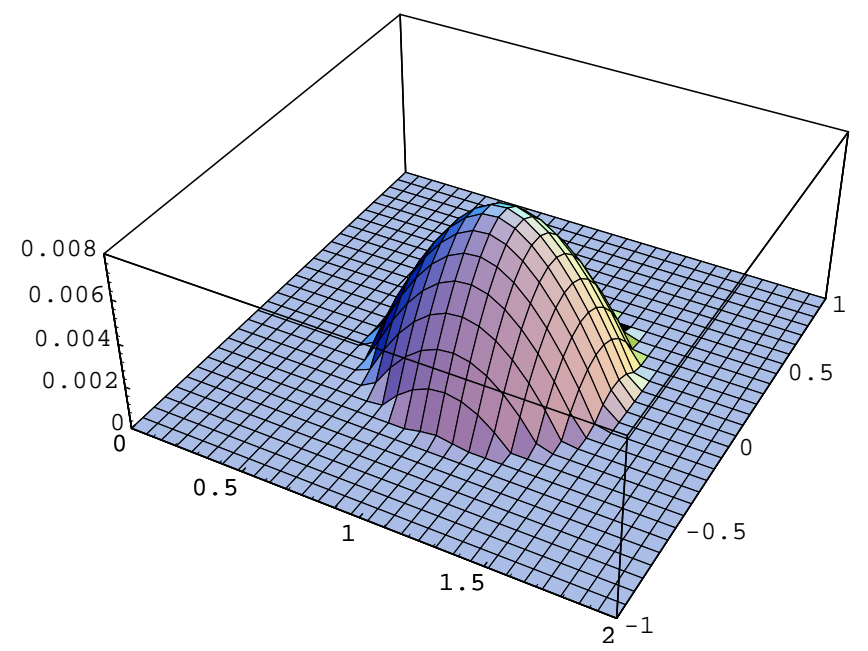

FIG. 3. The monopole condensate $\rho^{2}$, at the monopole region $u \sim \Lambda^{2}$, for $f_{0}=\Lambda / 10$.

Notice that $b_{11}$ diverges logarithmically at the singularities $u= \pm \Lambda^{2}$, but the corresponding local variable $a$ vanishes linearly at $u= \pm \Lambda^{2}$. It implies that $b_{11}|a|^{2} \rightarrow 0$ for $u \rightarrow \pm \Lambda^{2}$. It can be shown that the Seiberg-Witten solution gives $b_{01} \sim \Lambda / 8 \pi$ for $u \sim \Lambda$. It means that the monopole condenses at the monopole region (see fig. 3), since from the expression of the effective potential (XXIV.5), such condensation is energetically favoured. If we look at the dyon region, we find that $b_{01} \rightarrow 0$ for $u \rightarrow-\Lambda^{2}$. Numerically, there is a very small dyon condensate without any associated minimum in the effective potential in that region. On the other hand, there is a clear absolute minimum in the monopole region (see fig. (4). The different behaviors of the broken theory under the transformation $u \rightarrow-u$ is an expected result if we take into account that $f_{0} \neq 0$ breaks explicitly the $U(1)_{R}$ symmetry. 


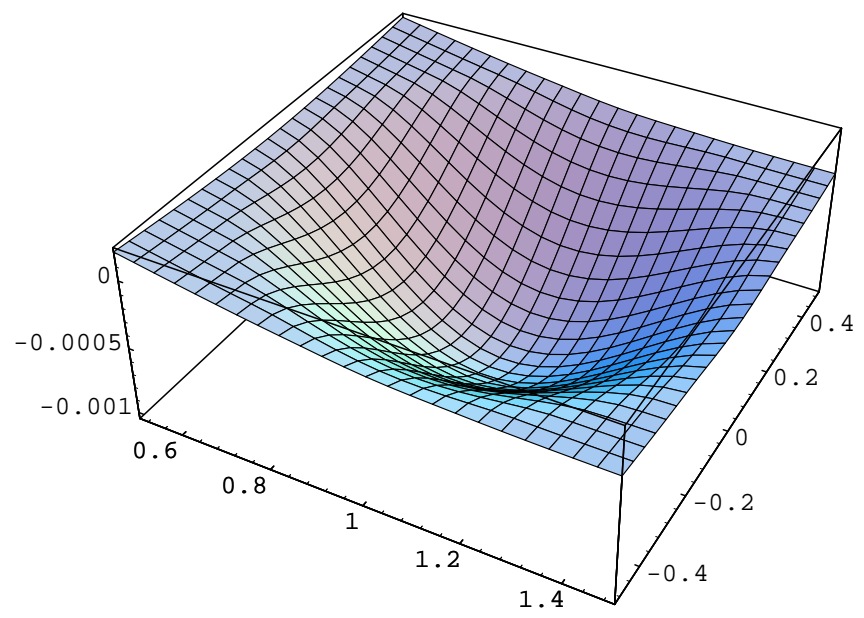

FIG. 4. The effective potential $V_{\text {eff }}(u)$ (XXIV.5), at the monopole region $u \sim \Lambda^{2}$, for $f_{0}=\Lambda / 10$.

The softly broken theory selects a unique minimum at the monopole region, with a non vanishing expectation value for the monopole. The theory confines and has a mass gap or order $\left(f_{0} \Lambda\right)^{1 / 2}$.

\section{STRING THEORY IN PERTURBATION THEORY.}

String Theory is a multifaceted subject. In the sixties strings were first introduced to model the dynamics of hadron dynamics. In section VII we described the confining phase as the dual Higgs phase, where magnetic degrees of freedom condense. The topology of the gauge group allows the existence of electric vortex tubes, ending on quark-antiquark bound states. The transverse size of the electric tubes is of the order of the compton wave length of the 'massive' W-bosons. At large distances, these electric tubes can be considered as open strings with a quark and an anti-quark at their end points. This is the QCD string, with an string tension of the order of the characteristic length square of the hadrons, $\alpha^{\prime} \sim(1 \mathrm{GeV})^{-2}$.

But the major interest in String Theory comes from being a good candidate for quantum gravity [48]. The macroscopic gravitational force includes an intrinsic constant, $G_{N}$, with dimensions of length square

$$
G_{N}=l_{p}^{2}=\left(1.6 \times 10^{-33} \mathrm{~cm}\right)^{2} .
$$

$(\mathrm{XXV} .1)$

In a physical process with an energy scale $E$ for the fundamental constituents of matter, the strength of the gravitational interaction is given by the dimensionless coupling $G_{N} E^{2}$ to the graviton. This interaction can be neglected when the graviton probes length scales much larger than the Planck's size, $G_{N} E^{2} \ll 1$. The interaction is also non-renormalizable. From the point of view of Quantum Field Theory, it corresponds to an effective low energy interaction, with $l_{p}$ the natural length scale at which the effects of quantum gravity become important. The natural suspicion is that there is new physics at such short distances, which smears out the interaction. The idea of String Theory is to replace the point particle description of the interactions by one-dimensional objects, strings with size of the order of the Planck's length $l_{p} \sim 10^{-33} \mathrm{~cm}$ (see fig. 5). Such simple change has profound consequences on the physical behavior of the theory, as we will briefly review below. It is still not clear whether the stringy solution to quantum gravity should work. Because Planck's length scale is so small, up to now String Theory is only constructed from internal consistency. But it is at the moment the best candidate we have. Let us quickly review some of the major implications of String Theory, derived already at perturbative level.

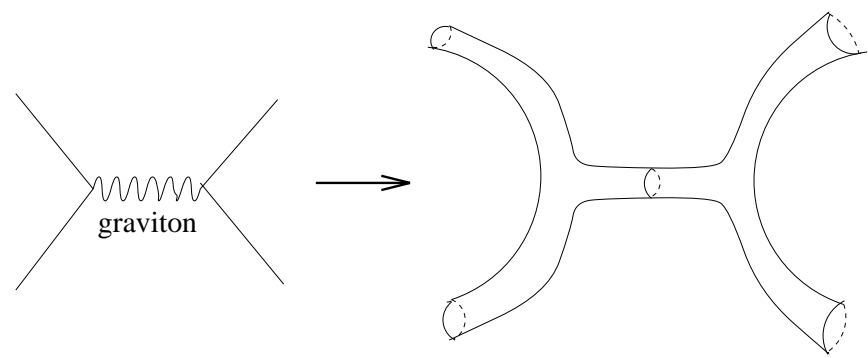

FIG. 5. The point particle graviton interchange is replaced by the smeared string interaction.

The first important consequence of String Theory is the existence of vibrating modes of the string. They correspond to the physical particle spectrum. For phenomenology the relevant part comes from the massless modes, since the massive modes are excited at energies of the order of the Planck's mass $l_{p}^{-1}$. At low energies all the massive modes decouple and we end with an effective Quantum Field Theory for the massless modes. In the massless spectrum of the closed string, there is a particle of spin two. It is the graviton. Then String Theory includes gravity. If we know how to make a consistent and phenomenologically satisfactory quantum theory of strings, we have quantized gravity.

Up to now, String Theory is only well understood at the perturbative level. The field theory diagrams are replaced by two dimensional Riemann surfaces, with the loop expansion being performed by an expansion in the genus of the surfaces. It is a formulation of first quantization, where the path integral is weighed by the area of the Riemann surface and the external states are included by the insertion of the appropiate vertex operators (see fig. 6). The perturbative string coupling constant is determined by the vacuum expectation value of a massless real scalar field, called the dilaton, through the relation $g_{s}=\exp \langle s\rangle$. The thickening of Feynman diagrams into 'surface' diagrams improves considerably the ultraviolet behavior of the theory. String Theory is ultraviolet finite. 


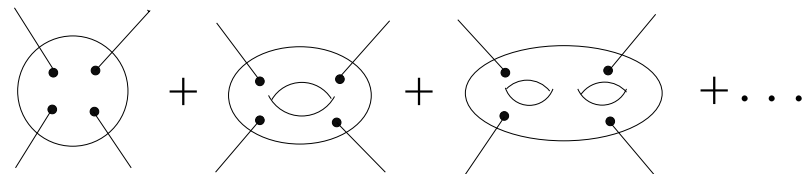

FIG. 6. The preturbative loop expansion in String Theory is equivalent to expand in the number of genus of the Riemann surfaces.

The third important consequence is the introduction of supersymmetry. For the bosonic string, the lowest vibrating mode correponds to a tachyon. It indicates that we are performing perturbation theory arround an unestable minimum. Supersymmetry gives a very economical solution to this problem. In a supersymmetric theory the hamiltonian operator is positive semi-definite and the ground state has always zero energy. It is also very appealing from the point of view of the cosmological constant problem. Furthermore, supersymmetry also introduces fermionic degrees of freedom in the physical spectrum. If nature really chooses to be supersymmetric at sort distances, the big question is: How is supersymmetry dynamically broken? The satisfactory answer must include the observed low energy phenomena of the standard model and the vanishing of the cosmological constant. As a last comment on supersymmetry we will say that the Green-Schwarz formulation of the superstring action demands invariance under a world-sheet local fermionic symmetry, called $\kappa$-symmetry. It is only possible to construct $\kappa$-symmetric world-sheet actions if the number of spacetime symmetries is $N \leq 2$ (in ten spacetime dimensions).

The fourth important consequence is the prediction on the number of dimensions of the target space where the perturbative string propagates. Lorentz invariance on the target space or conformal invariance on the worldsheet fixes the number of spacetime dimensions (twentysix for bosonic strings and ten for superstrings). As our low energy world is four dimensional, String Theory incorporates the Kaluza-Klein idea in a natural way. But again the one-dimensional nature of the string gives a quite different behavior of String Theory with respect to field theory. The dimensional reduction of a field theory in $D$ spacetime dimensions is another field theory in $D-1$ dimensions. The effect of a non-zero finite radius $R$ for the compactified dimension is just a tower of KaluzaKlein states with masses $n / R$. But in String Theory, the string can wind $m$ times around the compact dimension. This process gives a contribution to the momentum of the string proportional to the compact radius, $m R / \alpha^{\prime}$. These quantum states become light for $R \rightarrow 0$. The dimensional reduction of a String Theory in $D$ dimensions is another String Theory in $D$ dimensions. This is $T$ duality 49.

The fifth important consequence comes from the cancellation of spacetime anomalies (gauge, gravitational and mixed anomalies). It gives only the following five anomaly-free superstring theories in ten spacetime di- mensions.

\section{A. The type IIA and type IIB string theories.}

A type II string theory is constructed from closed superstrings with $N=2$ spacetime supersymmetries. The spectrum is obtained as a tensor product of a leftand right-moving world-sheet sectors of the closed string. Working in the light-cone gauge, the massless states of each sector are in the representation $\boldsymbol{8}_{v} \oplus \boldsymbol{8}_{ \pm}$of the little group $S O(8)$. The representations $\mathbf{8}_{v}$ and $\mathbf{8}_{ \pm}$are the vector representation and the irreducible chiral spinor representations of $S O(8)$, respectively.

The type IIA string theory corresponds to the choice of opposite chiralities for the spinorial representations in the left- and right-moving sectors,

$$
\text { Type IIA : } \quad\left(\mathbf{8}_{v} \oplus \mathbf{8}_{+}\right) \otimes\left(\mathbf{8}_{v} \oplus \mathbf{8}_{-}\right) .
$$

The bosonic massless spectrum is divided between the NS-NS fields:

$$
\mathbf{8}_{v} \otimes \mathbf{8}_{v}=\mathbf{1} \oplus \mathbf{2 8} \oplus \mathbf{3 5},
$$

which corresponds to the dilaton $s$, the antisymmetric tensor $B_{\mu \nu}$ and the gravitation field $g_{\mu \nu}$, respectively, and the R-R fields:

$$
\mathbf{8}_{+} \otimes \mathbf{8}_{-}=\mathbf{8}_{v} \oplus \mathbf{5 6}
$$

which correspond to the light-cone degrees of freedom of the antisymmetric tensors $A_{\mu}$ and $A_{\mu \nu \rho}$, respectively. As the chiral spinors have opposite chiralities, in the vertex operators of the R-R fields only even forms appear, $F_{2}$ and $F_{4}$. The physical state conditions on the massless states give the following equations on these even forms:

$$
d F=0 \quad d \star F=0,
$$

with $\star F$ the Poincare dual $(10-n)$-form of the $n$-form $F_{n}$. These are the Bianchi identity and the equation of motion for a field strength. Their relation with the R-R fields is then $F_{n}=d A_{n-1}$. The Abelian field strengths $F_{n}$ are gauge invariant, and since these are the fields that appear in the vertex operators, the fundamental strings do not carry RR charges.

The fermionic massless spectrum is given by the $N S-$ $R$ and $R-N S$ fields:

$$
\begin{gathered}
\mathbf{8}_{v} \otimes \mathbf{8}_{-}=\mathbf{8}_{+} \oplus \mathbf{5 6} \mathbf{6}_{-}, \\
\mathbf{8}_{+} \otimes \mathbf{8}_{v}=\mathbf{8}_{-} \oplus \mathbf{5} \mathbf{6}_{+} .
\end{gathered}
$$

The $\mathbf{8}_{ \pm}$states are the two dilatini. The $\mathbf{5 6}_{ \pm}$states are the two gravitini, with a spinor and a vector index. Observe that the fermions have opposite chiralities, which prevent the type IIA theory from gravitational anomalies.

The Type IIB String Theory corresponds to the choice of the same chirality for the spinor representations of the left- and right-moving sector, 


$$
\text { Type IIB : } \quad\left(\mathbf{8}_{v} \oplus \mathbf{8}_{+}\right) \otimes\left(\mathbf{8}_{v} \oplus \mathbf{8}_{+}\right) .
$$

The NS-NS fields are the same as for the type IIA string. The difference comes from the R-R fields:

$$
\mathbf{8}_{+} \otimes \mathbf{8}_{+}=\mathbf{1}+\oplus \mathbf{2 8} \oplus \mathbf{3 5} \mathbf{5}_{+} .
$$

They correspond, respectively, to the forms $A_{0}, A_{2}$ and $A_{4}$ (self-dual).

For the massless fermions there are two dilatini and two gravitini, but now all of them have the same chirality. In spite of it, the theory does not have gravitational anomalies [50].

Under spacetime compactifications, the type IIA and the type IIB string theories are unified by the $T$-duality symmetry. It is an exact symmetry of the theory already at the perturbative level and maps a type IIA string with a compact dimension of radius $R$ to a type IIB string with radius $\alpha^{\prime} / R$.

\section{B. The Type I string theory.}

It is constructed from unoriented open and closed superstrings, leading only $N=1$ spacetime supersymmetry. The massless states are:

$$
\begin{aligned}
& \text { Open : } \quad \mathbf{8}_{v} \otimes \mathbf{8}_{+} \\
& \text {Closed sym. : } \quad\left[\left(\mathbf{8}_{v} \oplus \mathbf{8}_{+}\right) \otimes\left(\mathbf{8}_{v} \oplus \mathbf{8}_{+}\right)\right]_{\text {sym }}= \\
& \quad=[\mathbf{1} \oplus \mathbf{2 8} \oplus \mathbf{3 5}]_{\text {bosonic }} \oplus\left[\mathbf{8}_{-} \oplus \mathbf{5} \mathbf{6}_{-}\right]_{\text {fermionic }} .
\end{aligned}
$$

The massless sector of the spectrum that comes from the unoriented open superstring (XXV.9) gives $N=1 \mathrm{su}-$ per Yang-Mills theory, with a gauge group $S O\left(N_{c}\right)$ or $U S p\left(N_{c}\right)$ introduced by Chan-Paton factors at the ends of the open superstring. The sector coming from the unoriented closed string (XXV.10) gives $N=1$ supergravity. Cancellation of spacetime anomalies restricts the gauge group to $S O(32)$.

\section{The $S O(32)$ and $E_{8} \times E_{8}$ heterotic strings.}

The heterotic string is constructed from a rightmoving closed superstring and a left-moving closed bosonic string. Conformal anomaly cancellation demands twenty-six bosonic target space coordinates in the left-moving sector. The additional sixteen left-moving coordinates $X_{L}^{I}, I=1, \ldots, 16$, are compactified on a $T^{16}$ torus, defined by a sixteen-dimensional lattice, $\Lambda_{16}$, with some basis vectors $\left\{e_{i}^{I}\right\}, i=1, \ldots, 16$. The left-moving momenta $p_{L}^{I}$ live on the dual lattice $\widetilde{\Lambda}_{16}$. The mass operator gives an even lattice $\left(\sum_{I=1}^{16} e_{i}^{I} e_{i}^{I}=2\right.$ for any $\left.i\right)$. The modular invariance of the one-loop diagrams restricts the lattice to be self-dual $\left(\widetilde{\Lambda}_{16}=\Lambda_{16}\right)$. There are only two even self-dual sixteen-dimensional lattices. They correspond to the root lattices of the Lie groups $S O(32) / Z_{2}$ and $E_{8} \times E_{8}$.
For the physical massless states, the supersymmetric right-moving sector gives the factor $\mathbf{8}_{v} \otimes \mathbf{8}_{+}$, which together with the lattice points of length squared two of the left-moving sector, give an $N=1$ vector multiplet in the adjoint representation of the gauge group $S O(32)$ or $E_{8} \times E_{8}$.

There is also a $T$-duality symmetry relating the two heterotic strings.

\section{D-BRANES.}

Perturbation theory is not the whole history. In the field theory sections we have learned how much the nonperturbative effects could change the perturbative picture of a theory. In particular, there are nonperturbative stable field configurations (solitons) that can become the relevant degrees of freedom in some regime. In that situation it is convenient to perform a duality transformation to have an effective description of the theory in terms of these solitonic degrees of freedom as the fundamental objects.

What about the nonperturbative effects in String Theory? Does String Theory incorporate nonperturbative excitations (string solitons)?. Are there also strong-weak coupling duality transformations in String Theory? Before the role of D-branes in String Theory were appreciated, the answers to these three questions were not clear.

For instance, it was known, by the study of large orders of string perturbation theory, that the nonperturbative effects in string theory had to be stronger than in field theory, in the sense of being of the order of $\exp \left(-1 / g_{s}\right)$ instead of order $\exp \left(-1 / g_{s}^{2}\right)$ [51, but it was not known which were the nature of such nonperturbative effects.

With respect the existence of nonperturbative objects, the unique evidence came form solitonic solutions of the supergravity equations of motion which are the low energy limits of string theories. These objects were in general extended membranes in $p+1$ dimensions, called $p$ branes [52].

In relation to the utility of the duality transformation in String Theory, there is strong evidence of some string dualities [53]. There is for instance the $S L(2, \mathbf{Z})$ selfduality conjecture of the type IIB theory 54. Under an $S$-transformation,the string coupling value $g_{s}$ is mapped to the value $1 / g_{s}$, and the NS-NS field $B_{\mu \nu}$ is mapped to the R-R field $A_{\mu \nu}$. Then, self-duality of type IIB demands the existence of an string with a tension scaling as $g_{s}^{-1}$ and non-zero RR charge.

\section{A. Dirichlet boundary conditions.}

In open string theory, it is possible to impose two different boundary conditions at the ends of the open string:

$$
\begin{aligned}
\text { Neuman : } & \partial_{\perp} X^{\mu} & =0 . \\
\text { Dirichlet }: & \partial_{t} X^{\mu} & =0 .
\end{aligned}
$$


An extended topological defect with $p+1$ dimensions is described by the following boundary conditions on the open strings:

$$
\partial_{\perp} X^{0,1, \cdots p}=\partial_{t} X^{p+1, \cdots 9}=0 .
$$

We call it a D $p$-brane (for Dirichlet 55]), an extended $(\mathrm{p}+1)$-dimensional object (located at $X^{p+1, \cdots 9}=$ const) with the end points of open strings attached to it.

The Dirichlet boundary conditions are not Lorentz invariant. There is a momentum flux going from the ends of open strings to the D-branes to which they are attached. In fact, the quantum fluctuations of the open string endpoints in the longitudinal directions of the D-brane live on the world-volume of the D-brane. The quantum fluctuations of the open string endpoints in the transverse directions of the D-brane, makes the D-brane fluctuate locally. It is a dynamical object, characterized by a tension $T_{p}$ and a $\mathrm{RR}$ charge $\mu_{p}$. If $\mu_{p} \neq 0$, the world-volume of a $p$-brane will couple to the $\mathrm{R}$-R $(p+1)$-form $A_{p+1}$.

Far from the D-brane, we have closed superstrings, but the world-sheet boundaries XXVI.3 relates the rightmoving supercharges to the left-moving ones, and only a linear combination of both is a good symmetry of the given configuration. In presence of the D-brane, half of the supersymmetries are broken. The D-brane is a BPS state. In fact, in [56] it was shown that the D-brane tension arises from the disk and therefore that it scales as $g_{s}^{-1}$. This is the same coupling constant dependence as for BPS solitonic branes carrying RR charges [52].

The Dirichlet boundary condition becomes the Neuman boundary condition in terms of the $T$-dual coordinates, and vice versa. It implies that if we $T$-dualize a direction longitudinal to the world- volume of the $\mathrm{D}$ $p$-brane, it becomes a $(p-1)$-brane. Equally, if the $T$ dualized direction is transverse to the $\mathrm{D} p$-brane, we obtain a $\mathrm{D}(p+1)$-brane. Consider a 9-brane in a type IIB background. The 9-brane fills the spacetime and the endpoints of the open strings attached to it are free to move in all the directions. It is a type I theory, with only $N=1$ supersymmetry. Now $T$-dualize one direction of the target space. We obtain an 8-brane in a type IIA background. If we proceed further, we obtain that a type IIB background can hold $p=9,7,5,3,1,-1 p$ branes. A D (-1)-brane is a D-instanton, a localized spacetime point. For a type IIA background we obtain $p=8,6,4,2,0$-branes.

\section{B. BPS states with RR charges.}

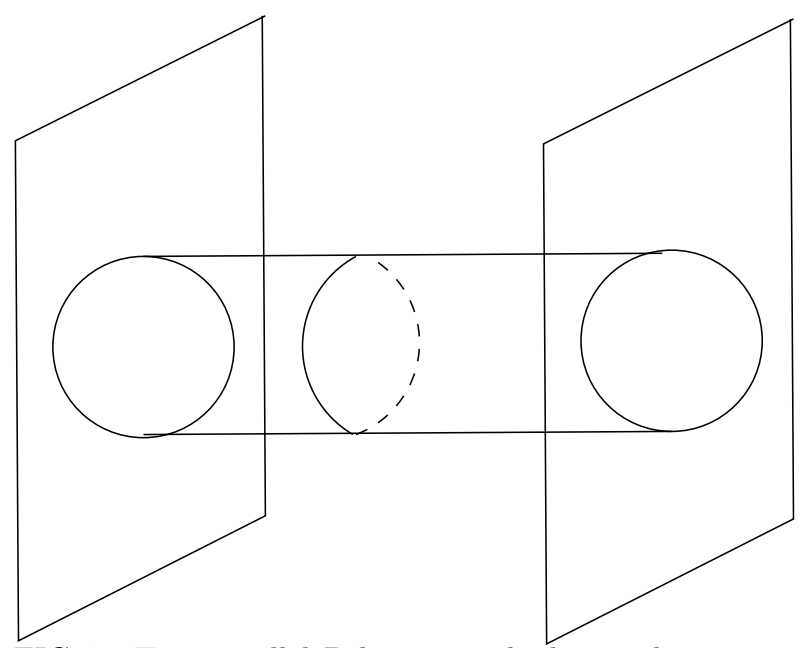

FIG. 7. Two parallel D-branes with the one-loop vacuum fluctuation of an open string attached between them. By modular invariance, it also corresponds to a tree level interchange of a closed string.

To check if really the D-branes are the nonperturbative string solitons required by string duality, Polchinski computed explicitly the tension and RR charge of a D $p$-brane 57. He first computed the one-loop amplitude of an open string attached to two parallel D $p$-branes. The resulting Casimir force between the D-branes was zero, supporting its BPS nature. By modular invariance, it can also be interpreted as the amplitude for the interchange of a closed string between the D-branes (see fig. 7). In the large separation limit, only the massless closed modes contribute. These are the NS-NS fields (graviton and dilaton) and the $\mathrm{R}-\mathrm{R}(p+1)$ form. On the space between the D-branes these fields follow the low energy type II action (type IIA for $p$ even and type IIB for $p$ odd). On the D $p$-branes, the coupling to the NS-NS and $\mathrm{R}$-R fields is

$$
S_{p}=T_{p} \int d^{p+1} \xi e^{-s}\left|\operatorname{det} G_{a b}\right|^{1 / 2}+\mu_{p} \int_{p-\text { brane }} A_{p+1} .
$$

(XXVI.4)

From (XXVI.4) we see that the actual D-brane action includes a dilaton factor $\tau_{p}=T_{p} / g_{s}$, with $g_{s}$ the coupling constant of the closed string theory. Comparing the field theory calculation with the contribution of the massless closed modes in the string theory computation, one can obtain the values of $T_{p}$ and $\mu_{p}$. The result is 57]

$$
\mu_{p}^{2}=2 T_{p}^{2}=\left(4 \pi^{2} \alpha^{\prime}\right)^{3-p}
$$

Observe that the R-R charge is really non-zero. In fact, if one checks (the generalization of) the Dirac's quantization condition for the charge $\mu_{p}$ and its dual charge $\mu_{(6-p)}$, one obtains that $\mu_{p} \mu_{(6-p)}=2 \pi$. They satisfy the minimal quantization condition. It means that the D-branes carry the minimal allowed RR charges. 


\section{SOME FINAL COMMENTS ON NONPERTURBATIVE STRING THEORY.}

A. D-instantons and S-duality.

The answers to the three questions at the beginning of the previous section can now be more concrete, since some nonperturbative objects in String Theory has been identified: the D-branes.

Consider a D $p$-brane wrapped around a non-trivial $(p+1)$-cycle. This configuration is topologically stable. Its action is $T_{p} V_{p+1} / g_{s}$, with $V_{p+1}$ the volume of the nontrivial $(p+1)$ cycle. It contributes in amplitudes with factors $e^{-T_{p} V_{p+1} / g_{s}}$, a generalized instanton effect. Now we understand why the nonperturbative effects in String Theory are stronger than in field theory, it is related to the peculiar nature of the string solitons.

The D-branes also give the necessary ingredient for the $S L(2, \mathbf{Z})$ self-duality of the type IIB string theory. This theory allows $D 1$-branes, with a mass $\tau_{1} \sim\left(2 \pi \alpha^{\prime} g_{s}\right)^{-1}$ in the string metric and non-zero RR charge. Also, one can see that on the D 1-brane there are the same fluctuations of a fundamental IIB string [58]. Then, it is the required object for the $S$-duality transformation of the type IIB string. In fact, at strong coupling the D 1-string becomes light and it is natural to formulate the type IIB theory in terms of weakly coupled D 1-branes.

There is another $S$-duality relation in String Theory. Observe that the type I theory and the $S O(32)$ heterotic theory have the same low energy limit. It could be that they correspond to the same theory but for different values of the string coupling constant. Again D-branes help to make this picture clearer. Consider a D 1-brane in a type I background with open strings attached to it, but also with open strings with one end point attached to a 9-brane. We call them $1-9$ strings. The 9-brane fills the spacetime, and the $1-9$ strings, having one ChanPaton index, are vectors of $S O(32)$. One can see that the world-sheet theory of the D 1-brane is precisely that of the $S O(32)$ heterotic string [59]. Having a tension that scales as $g_{s}^{-1}$, one can argue that this $\mathrm{D}$ heterotic string sets the lightest scale in the theory when $g_{s} \gg 1$. The strong coupling behavior of the type I string can be modeled by the weak coupling behavior of the heterotic string.

\section{B. An eleventh dimension.}

Type IIA allows the existence of 0-branes that couple to the R-R one-form $A_{1}$. The 0-brane mass is $\tau_{0} \sim\left(\alpha^{\prime}\right)^{-1 / 2} / g_{s}$ in the string metric. At strong coupling in the type IIA theory, $g_{s} \gg 1$, this mass is the lightest scale of the theory. In fact, $n$ 0-branes can form a BPS bound state with mass $n \tau_{0}$. This tower of states becoming a continuum of light states at strong coupling is characteristic of the appearance of an additional dimension. Type IIA theory at strong coupling feels an eleventh dimension of some size $2 \pi R$, with the 0 -branes playing the role of the Kaluza-Klein states [60].

If we compactify 11D supergravity 61 on a circle of radius $R$ and compare its action with the 10D type IIA supergravity action, we obtain the relation

$$
R \sim g_{s}^{2 / 3}
$$

(XXVII.1)

This eleventh dimension is invisible in perturbation theory, where we perform an expansion near $g_{s}=0$.

This has been a lightning review of some aspects of duality in String Theory. We hope it will serve to whet the appetite of the reader and encourage her/him to learn more about the subject and to eventually contribute to some of the outstanding open problems. More information can be found from the references 62.

\section{Acknowledgments}

We have benefited from valuable conversations with many colleages. We would like to thank in particular E. Álvarez, J.M. F. Barbón, J. Distler, D. Espriu, C. Gómez, J. Gomis, K. Kounnas, J. Labastida, W. Lerche, M. Mariño, J.M. Pons and E. Verlinde for discussions. F. Z. would like to thank the Theory Division at CERN for its hospitality. The work of F. Z. is supported by a fellowship from Ministerio de Educación y Ciencia.

[1] S. Deser and C. Teitelboim, Phys. Rev. D13 (1976), 1592 ;

S. Deser, A. Gomberoff, M. Henneaux and C. Teitelboim, Phys. Lett. B400 (1997), 80.

[2] P.A.M. Dirac, Proc. Roy. Soc. A133 (1931), 60.

[3] J. Schwinger, Phys. Rev. 144 (1966) 1087; 173 (1968) 1536 ;

D. Zwanziger, Phys. Rev. 176 (1968) 1480.

[4] C. Vafa and E. Witten, Nucl. Phys. B431 (1994), 3; F. Ferrari, hep-th/9702166.

[5] H.B. Nielsen and P. Olesen, Nucl. Phys. B61(1973), 4561.

[6] G. 't Hooft, Nucl. Phys. B79 (1974) 276.

[7] A. M. Polyakov, JETP Lett. 20 (1974) 194.

[8] B. Julia and A. Zee, Phys. Rev. D11 (1975) 2227.

[9] E. B. Bogomol'nyi, Sov. J. Nucl. Phys. 24 (1976) 449.

[10] M. K. Prasad and C. M. Sommerfield, Phys. Rev. Lett. 35 (1975) 760.

[11] E. Witten, Phys. Lett. 86B (1979) 283.

[12] G. 't Hooft, Nucl. Phys. B190[FS3](181), 455, and 1981 Cargese Summer School Lecture Notes on Fundamental Interactions, in 'Under the Spell of the Gauge Principle', World Scientific, 1994. 
[13] N. Seiberg and E. Witten, Nucl. Phys. B426 (1994), 19.

[14] L. Álvarez-Gaumé, J. Distler, C. Kounnas and M. Mariño, Int. J. Mod. Phys. A11 (1996) 4745;

L. Álvarez-Gaumé and M. Mariño, Int. J. Mod. Phys. A12 (1997) 975.

[15] L. Álvarez-Gaumé, M. Mariño and F. Zamora, hepth/9703072, hep-th/9707017, to appear in Int. J. Mod. Phys. A.

[16] N. Evans, S.D.H. Hsu and M. Schwetz, Nucl. Phys. B484, 124 (1997).

[17] K.G. Wilson, Phys. Rev. D10 (1974), 2445.

[18] E. Fradkin and S.H. Shenker, Phys. Rev. D12 (1979), 3682 ;

T. Banks and E. Rabinovici, Nucl. Phys. B160 (1979), 349.

[19] J. Wess and J. Bagger, 'Supersymmetry and Supergravity', Princeton University Press, 2nd ed., 1992; and references therein.

[20] A. Salam and J. Strathdee, Nucl. Phys. B76 (1974), 477.

[21] S. Weinberg, 'The Quantum Theory of Fields I', Cambridge University Press, 1996.

[22] B. Zumino, Phys. Lett. 87B, 203 (1979).

[23] M.T. Grisaru, W. Siegel and M. Rocek, Nucl. Phys. B159 (1979), 429.

[24] N. Seiberg, 'The Power of Holomorphy: Exact results in 4-D SUSY Gauge Theories.', in PASCOS 94, pg.357, hep-th/9408013.

[25] K. Konishi and K. Shizuya, Nuovo Cim. 90A (1985), 111.

[26] N. Arkani-Hamed and H. Murayama, hep-th/9705189, hep-th/9707133.

[27] B. de Wit, P.G. Lauwers and A. van Proeyen, Nucl. Phys. B255 (1985), 569.

[28] K. Intriligator and N. Seiberg, Nucl. Phys. Proc. Suppl. 45BC (1996) 1, hep-th/9509066;

M.E. Peskin, TASI 96 lectures, hep-th/9702094;

M. Shifman, hep-th/9704114.

[29] E. Witten, Nucl. Phys. B202 (1982), 253.

[30] A.C. Davis, M. Dine and N. Seiberg, Phys. Lett. 125B (1983), 487;

I. Affleck, M. Dine and N. Seiberg, Phys. Rev. Lett 51, 1026 (1983).

[31] I. Affleck, M. Dine and N. Seiberg, Nucl. Phys. B241 (1984), 493.

[32] D. Finnell and P. Pouliot, Nucl. Phys. B453 (1995), 225.

[33] K. Intriligator, R.G. Leigh and N. Seiberg, Phys. Rev. D50 (1994), 1092.

[34] K. Intriligator, Phys. Lett. 336B (1994), 409.

[35] G. Veneziano and S. Yankielowicz, Phys. Lett. 113B (1982), 231; T. Taylor, G. Veneziano and S. Yankielowicz, Nucl. Phys. B218 (1983), 439.

[36] N. Seiberg, Phys. Rev. D49 (1994), 6857.

[37] G. 't Hooft, 'Naturalness, chiral symmetry, and spontaneous chiral symmetry breaking', Cargese 1979, in 'Under the Spell of the Gauge Principle', World Scientific, 1994.

[38] N. Seiberg, Nucl. Phys. B435 (1995), 129.

[39] T. Banks and A. Zaks, Nucl. Phys. B196 (1982), 189.

[40] R. Haag, J. T. Lopuszanski and M. Sohnius, Nucl. Phys. B88 (1975) 257.

[41] S.J. Gates, Jr., Nucl. Phys. B238 (1984), 349.

[42] L. Álvarez-Gaumé and S.F. Hassan, Fortsch. Phys. 45,
159 (1997);

A. Bilal, hep-th/9601077;

C. Gómez and R. Hernández, Advanced School on Effective Theories, Almuñecar 1995, hep-th/9510023.

W. Lerche, Nucl. Phys. Proc. Suppl. 55B, 83 (1997).

[43] E. Witten and D. Olive, Phys. Lett. 78B (1978) 97.

[44] N. Seiberg and E. Witten, Nucl. Phys. B431 (1994), 484.

[45] S. Mandelstam, Phys. Rept. C23 (1976), 245.

[46] L. Girardello and M.T. Grisaru, Nucl. Phys. B194 (1982), 65.

[47] N. Evans, S.D.H. Hsu and M. Schwetz, Phys. Lett 355B 475 (1995), Nucl. Phys. B 456205 (1995);

O. Aharony, J. Sonnenschein, M.E. Peskin and S. Yankielowicz, Phys. Rev. D52 6157 (1995);

E. D'Hoker, Y. Mimura and N. Sakai, Phys. Rev. D54 7724 (1996).

[48] M.B. Green, J.H. Schwarz and E.Witten, 'Superstring Theory', Cambridge University Press (1987); and references therein.

[49] A. Giveon, M. Porrati and E. Rabinovici, Phys. Rept. 244, 77 (1994);

and references therein.

[50] L. Álvarez-Gaumé and E. Witten, Nucl. Phys. B234, 269 (1984).

[51] S.H. Shenker, 'The Strength of Nonperturbative Effects in String Theory', in Random Surfaces and Quantum Gravity, eds. O.Alvarez et al. (1991).

[52] M.J. Duff, R.R. Khuri and J.X. Lu, Phys. Rept. 259, 213 (1995);

M.J. Duff, 'Supermembranes', TASI 96 lectures, hepth/9611203, and references therein.

[53] C.M. Hull and P.K. Townsend, Nucl. Phys. B438(1995), 109 ;

E. Witten, Nucl. Phys. B443(1995), 83;

A. Strominger, Nucl. Phys. B451(1995), 96.

[54] A. Font, L. Ibañez, D. Lust and F. Quevedo, Phys. Lett. 249, 35 (1990).

[55] J. Polchinski, TASI 96 lectures, hep-th/9611050; C. Bachas, 'Half a Lecture on D-branes', hep-th/9701019; and references therein.

[56] J. Dai, R.G. Leigh and J. Polchinski, Mod. Phys. Lett. A4, 2073 (1989);

R.G. Leigh, Mod. Phys. Lett. A4, 2767 (1989).

[57] J. Polchinski, Phys. Rev. Lett. 75 (1995), 4724.

[58] E. Witten, Nucl. Phys. B460 (1996), 335.

[59] J. Polchinski and E. Witten, Nucl. Phys. B460 (1996), 525.

[60] P.K. Townsend, Phys. Lett. B350 (1995), 184;

E. Witten, Nucl. Phys. B443 (1995), 85.

[61] E. Cremmer, B. Julia and J. Scherk, Phys. Lett. 76B, 409.

[62] P. Aspinwall, TASI 96 lectures, hep-th/9611137;

J. Schwarz, TASI 96 lectures, hep-th/9607202;

P.K. Townsend, Trieste 95 lectures, hep-th/9612121;

E. Witten, Nucl. Phys. B460 (1996) 335;

P. Horava and E. Witten, Nucl. Phys. B460 (1996) 506; T. Banks, W. Fischler, S.H. Shenker and L. Susskind, Phys. Rev. D55 5112 (1997);

A. Hanany and E. Witten, Nucl. Phys. B492 (1997) 152; 
E. Witten, hep-th/9703166;

R. Dijkgraaf, E. Verlinde and H. Verlinde, hep-

th/9709107. 